\title{
Mechanism of Reversible Alkyne Coupling at Zirconocene: Ancillary Ligand Effects
}

\author{
Adam D. Miller, Jennifer L. McBee, and T. Don Tilley* \\ Contribution from the Department of Chemistry, University of California, Berkeley, Berkeley, \\ California 94720-1460
}

Experimental Methods

Table S1: Rate constants at varying ratios of [PMe 3 :[Zirconacycle]

Figure S1: Plot of $k_{\mathrm{obs}}$ versus [PMe 3$] /[$ Zirconacycle]

Table S2: Rate constants at varying ratios of $\left[\mathrm{PhC} \equiv \mathrm{CSiMe}_{3}\right]:\left[\mathrm{PMe}_{3}\right]$

Figure S2: Plot of $k_{\mathrm{obs}}$ versus $\left[\mathrm{PhC} \equiv \mathrm{CSiMe}_{3}\right] /\left[\mathrm{PMe}_{3}\right]$

S4

X-Ray Structural Data for 1

S5-S15

X-Ray Structural Data for 2

S15-S26

References 


\section{Experimental Methods}

Influence of $\left[\mathrm{PhC} \equiv \mathrm{CSSiMe}_{3}\right]$ on the Rate of $\mathrm{Cp}_{2} \mathrm{Zr}\left[2,5-\left(\mathrm{Me}_{3} \mathrm{Si}\right)_{2}-3,4-\mathrm{Ph}_{2} \mathrm{C}_{4}\right]$ (9) Fragmentation: Zirconacyclopentadiene 9 (ca. $4 \mathrm{mg}, 7 \mu \mathrm{mol}$ ) and ferrocene (ca. $2 \mathrm{mg}, 10.7 \mu \mathrm{mol}$ ) were dissolved in benzene- $d_{6}$ and then loaded into a Teflon-sealed NMR tube. To this solution was added $\mathrm{PMe}_{3}\left(10\right.$ equivs, ca. $7 \mu \mathrm{L}, 70 \mu \mathrm{mol}$ ) and this was then treated with $\mathrm{PhC} \equiv \mathrm{CSiMe}_{3}$ (four different $\left[\mathrm{PhC} \equiv \mathrm{CSiMe}_{3}\right]$ were examined: 30 equivs, $0.18 \mathrm{mmol}, 35 \mu \mathrm{L}$; 90 equivs, $0.45 \mathrm{mmol}, 88$ $\mu \mathrm{L}$; 420 equivs, $2.1 \mathrm{mmol}, 0.45 \mathrm{~mL} ; 550$ equivs, $2.7 \mathrm{mmol}, 0.50 \mathrm{~mL}$ ). Note: As the amount of $\mathrm{PhC} \equiv \mathrm{CSiMe}_{3}$ increased, less benzene- $d_{6}$ was added to keep the volume of sample ca. $0.5 \mathrm{~mL}$ until the volume of $\mathrm{PhC} \equiv \mathrm{CSiMe}_{3}$ became greater than $0.5 \mathrm{~mL}$. At this point the samples had a greater volume.

Data points were gathered by ${ }^{1} \mathrm{H}$ NMR spectroscopy at $50{ }^{\circ} \mathrm{C}$, and the rate of disappearance of 9 was monitored by integrating the $-\mathrm{SiMe}_{3}$ peak relative to that of $\mathrm{Cp}_{2} \mathrm{Fe}$. Rate constants were calculated from first-order plots using data from the first three to five half-lives. 
Table S1: The observed first-order rate constants of zirconacycles $\mathrm{CpCp} * \mathrm{Zr}\left[2,5-\left(\mathrm{Me}_{3} \mathrm{Si}_{2}-3,4-\right.\right.$ $\left.\mathrm{Ph}_{2} \mathrm{C}_{4}\right](\mathbf{1}), \mathrm{Me}_{2} \mathrm{C}\left(\eta^{5}-\mathrm{C}_{5} \mathrm{H}_{4}\right)_{2} \mathrm{Zr}\left[2,5-\left(\mathrm{Me}_{3} \mathrm{Si}\right)_{2}-3,4-\mathrm{Ph}_{2} \mathrm{C}_{4}\right]$ (2), and $\mathrm{Cp}_{2} \mathrm{Zr}\left[2,5-\left(\mathrm{Me}_{3} \mathrm{Si}_{2}-3,4-\mathrm{Ph}_{2} \mathrm{C}_{4}\right]\right.$ (9) at various ratios of [ $\left.\mathrm{PMe}_{3}\right]$ :[Zirconacycle].

\begin{tabular}{cccc}
\hline Zirconacycle & ${\text { Temperature }\left({ }^{\circ} \mathbf{C}\right)}^{\left[\mathrm{PMe}_{3}\right] /[\text { Zirconacycle] }}$ & $\boldsymbol{k}_{\text {obs }} \times \mathbf{1 0 0 0}\left(\mathbf{s}^{-1}\right)$ \\
\hline $\mathbf{1}$ & 75 & 10 & $1.5(1)^{\mathrm{a}}$ \\
$\mathbf{1}$ & 75 & 16.5 & $1.6(2)^{\mathrm{b}}$ \\
$\mathbf{1}$ & 75 & 38 & $1.4(3)^{\mathrm{b}}$ \\
\hline $\mathbf{2}$ & 20 & 1.1 & $1.4(1)^{\mathrm{b}}$ \\
$\mathbf{2}$ & 20 & 2 & $1.45(7)^{\mathrm{b}}$ \\
$\mathbf{2}$ & 20 & 10 & $1.4(1)^{\mathrm{a}}$ \\
\hline $\mathbf{9}$ & 50 & 7.5 & $1.08(7)^{\mathrm{b}}$ \\
$\mathbf{9}$ & 50 & 14 & $1.09(6)^{\mathrm{b}}$ \\
$\mathbf{9}$ & 50 & 40 & $1.09(9)^{\mathrm{b}}$ \\
\hline
\end{tabular}

${ }^{a}$ average of three runs; ${ }^{b}$ single run

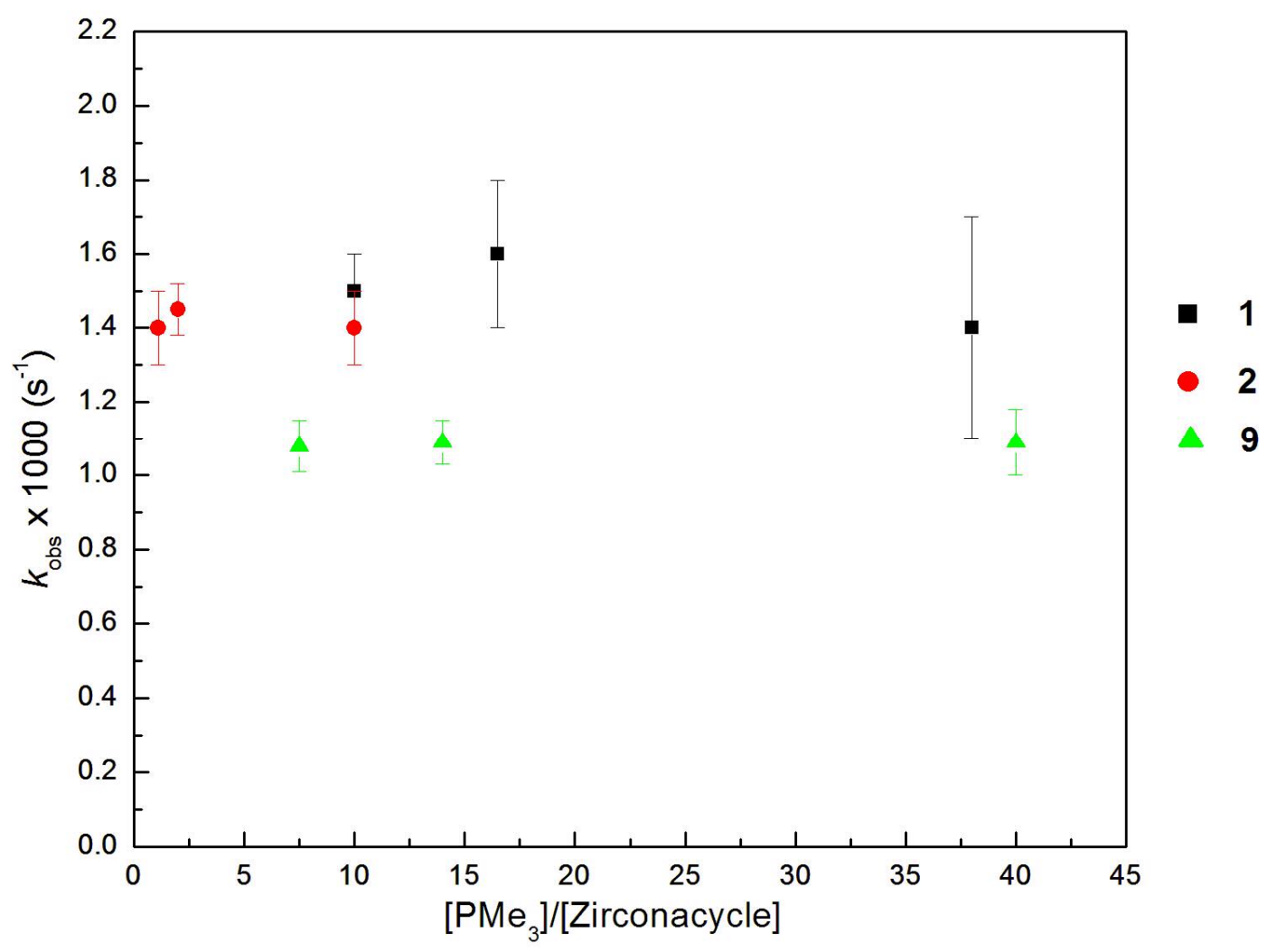

Figure S1. The observed first-order rate constants versus the ratio of [PMe $]$ :[Zirconacycle]. 
Table S2. The observed first-order rate constants measured at $50{ }^{\circ} \mathrm{C}$ for zirconacycle 9 at various ratios of $\left[\mathrm{PhC} \equiv \mathrm{CSiMe}_{3}\right]:\left[\mathrm{PMe}_{3}\right]$.

\begin{tabular}{cc}
\hline$\left[\mathrm{PhC} \equiv \mathrm{CSiMe}_{3}\right] /\left[\mathrm{PMe}_{3}\right]$ & $\boldsymbol{k}_{\text {obs }} \times \mathbf{1 0 0 0}\left(\mathbf{s}^{-1}\right)$ \\
\hline 3 & $0.8(3)$ \\
9 & $0.8(2)$ \\
42 & $1.4(3)$ \\
55 & $0.5(1)$ \\
\hline
\end{tabular}

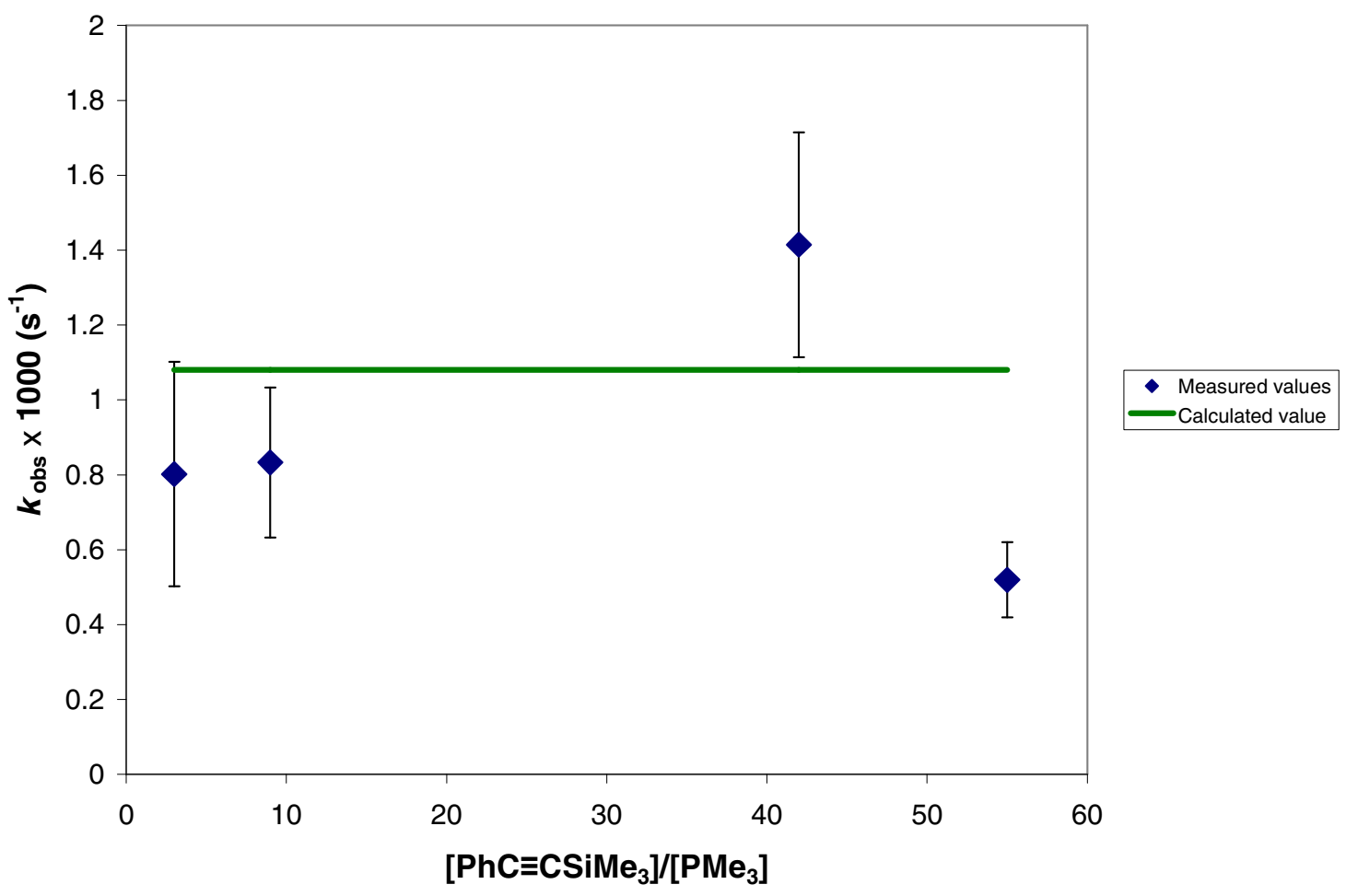

Figure S4. The observed first-order rate constants measured at $50{ }^{\circ} \mathrm{C}$ for zirconacycle 9 versus $\left[\mathrm{PhC} \equiv \mathrm{CSiMe}_{3}\right] /\left[\mathrm{PMe}_{3}\right]$. The calculated value is the observed rate constant measured without added $\mathrm{PhC} \equiv \mathrm{CSiMe}_{3}$ but under otherwise similar conditions $\left(k_{\mathrm{obs}}=1.08 \times 10^{-3} \mathrm{~s}^{-1}\right)$. 


\section{General Crystallographic Comments} angle data.

The diffrn_reflns_theta_full in each structure is lower than set by IUC due to the lack of high

\section{X-Ray Structural Data for 1:}

\section{Data Collection}

A fragment of a yellow block-like crystal of $\mathrm{C}_{37} \mathrm{H}_{48} \mathrm{Si}_{2} \mathrm{Zr}$ having approximate dimensions of $0.5 \times 0.3 \times 0.3 \mathrm{~mm}$ was mounted on a glass fiber using Paratone $\mathrm{N}$ hydrocarbon oil. All measurements were made on a Bruker P4 CCD area detector with graphite monochromated MoK radiation.

Cell constants and an orientation matrix, obtained from a least-squares refinement using the measured positions of 5942 centered reflections with $\mathrm{I}>10 \sigma(\mathrm{I})$ in the range $3.52<\theta<24.65^{\circ}$ corresponded to a P-centered triclinic cell with dimensions:

$$
\begin{array}{ll}
\mathrm{a}=11.508(1) \AA & \alpha=87.713(1)^{\mathrm{o}} \\
\mathrm{b}=11.569(1) \AA & \beta=69.630(1)^{\mathrm{o}} \\
\mathrm{c}=15.998(2) \AA & \gamma=60.151(1)^{\circ} \\
\mathrm{V}=8876(1) \AA^{3} &
\end{array}
$$

For $\mathrm{Z}=2$ and F.W. $=640.2$, the calculated density is $1.24 \mathrm{~g} / \mathrm{cm}^{3}$.

Analysis of the systematic absences allowed the space group to be uniquely determined to be: P-1

The data were collected at a temperature of 150(2) K. Frames corresponding to an arbitrary hemisphere of data were collected using $\omega$ scans of $0.3^{\circ}$ counted for a total of 10 seconds per frame.

\section{$\underline{\text { Data Reduction }}$}

Data were integrated by the program $\mathrm{SAINT}^{1}$ to a maximum $\theta$ value of $23.25^{\circ}$. The data were corrected for Lorentz and polarization effects. Data were analyzed for agreement and possible absorption using XPREP ${ }^{2}$. An empirical absorption correction based on comparison of redundant and equivalent reflections was applied using SADABS ${ }^{3} .\left(\mathrm{T}_{\max }=0.884, \mathrm{~T}_{\min }=0.810\right)$. Of the 7602 reflections that were collected, 4737 were unique $\left(\mathrm{R}_{\mathrm{int}}=0.0581\right)$; equivalent reflections were merged. No decay correction was applied.

\section{$\underline{\text { Structure Solution and Refinement }}$}

The structure was solved by direct methods ${ }^{1}$ and expanded using Fourier techniques ${ }^{4}$. Some non-hydrogen atoms were refined anisotropically, while the rest were refined isotropically. 
Hydrogen atoms were included but not refined. The final cycle of full-matrix least-squares refinement $^{5}$ was based on 4737 reflections (all data) and 361 variable parameters and converged (largest parameter shift was 0.031 times its esd) with conventional unweighted and weighted agreement factors of:

$\mathrm{R} 1=\Sigma|| \mathrm{F}_{\mathrm{o}}|-| \mathrm{F}_{\mathrm{c}} \| / \Sigma\left|\mathrm{F}_{\mathrm{o}}\right|=0.04032$ for 4185 data with $\mathrm{I}>2 \sigma(\mathrm{I})$

$\mathrm{wR} 2=\left[\left(\Sigma \mathrm{w}\left(\left|\mathrm{F}_{\mathrm{o}}\right|^{2}-\left|\mathrm{F}_{\mathrm{c}}\right|^{2}\right)^{2} / \Sigma \mathrm{w} \mid \mathrm{Fo}^{2}\right)\right]^{1 / 2}=0.1065$

The standard deviation of an observation of unit weight ${ }^{6}$ was 1.166 . The weighting scheme was based on counting statistics and included a factor to downweight the intense reflections. The maximum and minimum peaks on the final difference Fourier map corresponded to 0.560 and -0.561 $\mathrm{e}^{-} / \AA^{3}$, respectively.

Neutral atom scattering factors were taken from Cromer and Waber ${ }^{7}$. Anomalous dispersion effects were included in Fcalc ${ }^{8}$; the values for Df' and Df" were those of Creagh and McAuley ${ }^{9}$. The values for the mass attenuation coefficients are those of Creagh and Hubbel ${ }^{10}$. All calculations were performed using the SHELXTL ${ }^{11}$ crystallographic software package of Bruker Analytical X-ray Systems Inc.

\section{Experimental Details:}

\section{A. Crystal Data}

Empirical Formula

Formula Weight

Crystal Color, Habit

Crystal Dimensions

Crystal System

Lattice Type

Lattice Parameters

Space Group

$$
\begin{aligned}
& \mathrm{C}_{37} \mathrm{H}_{48} \mathrm{Si}_{2} \mathrm{Zr} \\
& 640.2 \\
& \text { yellow, block } \\
& 0.5 \times 0.3 \times 0.3 \mathrm{~mm} \\
& \text { Triclinic } \\
& \text { primitive, } \mathrm{P} \text {-centered } \\
& \mathrm{a}=11.508(1) \AA \\
& \mathrm{b}=11.569(1) \AA \\
& \mathrm{c}=15.998(2) \AA \\
& \alpha=87.713(1)^{\mathrm{O}} \\
& \beta=69.630(1)^{\mathrm{O}} \\
& \gamma=60.151(1)^{\mathrm{o}} \\
& \mathrm{V}=1708.4(3) \AA^{3} \\
& \mathrm{P}-1 \\
& 2 \\
& 1.24 \mathrm{~g} / \mathrm{cm}^{3} \\
& 0.42 \mathrm{~cm}^{-1}
\end{aligned}
$$$$
Z \text { value }
$$$$
\mathrm{D}_{\text {calc }}
$$$$
\mu(\mathrm{MoK})
$$ 
B. Intensity Measurements

Diffractometer

Radiation

Detector Position

Exposure Time

Scan Type

$\theta_{\max }$

No. of Reflections Measured

Corrections

C. Structure Solution and Refinement

Structure Solution

Refinement

Function Minimized

Least Squares Weighting scheme

Anomalous Dispersion

No. Observations $(\mathrm{I}>2.00 \sigma(\mathrm{I}))$

No. Variables

Residuals: R; $\mathrm{wR}_{2}$; Rall

Goodness of Fit Indicator

Max Shift/Error in Final Cycle

Maximum peak in Final Diff. Map

Minimum peak in Final Diff. Map
Bruker P4

$\operatorname{MoK}(\lambda=0.71073 \AA)$

graphite monochromated

$60.00 \mathrm{~mm}$

10 seconds per frame.

$\omega$ ( 0.3 degrees per frame)

$23.25^{\circ}$

Total: 7602

Unique: $4737\left(\mathrm{R}_{\text {int }}=0.0581\right)$

Lorentz-polarization

Absorption $\left(\mathrm{T}_{\max }=0.884\right.$,

$\mathrm{T}_{\min }=0.810$ ) direct (SHELXS-97 (Sheldrick, 1990))

Full-matrix least-squares

$\mathrm{R} w\left(\left|\mathrm{~F}_{\mathrm{o}}\right|^{2}-\left|\mathrm{F}_{\mathrm{c}}\right|^{2}\right)^{2}$

$\mathrm{w}=1 /\left[\sigma^{2}\left(\mathrm{~F}_{\mathrm{o}}^{2}\right)+(\mathrm{qP})^{2}+0.000 \mathrm{P}\right]$

where $\mathrm{P}=\left[\mathrm{F}_{\mathrm{o}}{ }^{2}+2 \mathrm{~F}_{\mathrm{c}}{ }^{2}\right] / 3$

All non-hydrogen atoms

4185

361

$0.0403 ; 0.1065 ; 0.0443$

1.166

0.031

$0.560 \mathrm{e}^{-} / \AA^{3}$

$-0.561 \mathrm{e}^{-} / \AA^{3}$

Table S3. Atomic coordinates and $\mathrm{U}_{\mathrm{iso}} / \mathrm{U}_{\mathrm{eq}}$ and occupancy for $\mathbf{1} . \mathrm{U}_{\mathrm{eq}}$ is defined as one third of the trace of the orthogonalized $\mathrm{U}_{\mathrm{ij}}$ tensor.

$\begin{array}{lcclll}\text { atom } & \mathbf{x} & \mathbf{y} & \mathbf{z} & \mathbf{U}_{\mathbf{e q}} & \text { Occupancy } \\ \text { Zr } & 0.7304(1) & 0.6916(1) & 0.7524(1) & 0.021(1) & 1 \\ \text { Si2 } & 1.0339(1) & 0.4015(1) & 0.8100(1) & 0.032(1) & 1 \\ \text { Si1 } & 0.5335(1) & 0.6507(1) & 0.6192(1) & 0.025(1) & 1 \\ \text { C10 } & 0.5127(3) & 0.7345(3) & 0.8938(2) & 0.026(1) & 1 \\ \text { C23 } & 0.7290(3) & 0.3479(3) & 0.6470(2) & 0.023(1) & 1 \\ \text { C1 } & 0.6500(3) & 0.5910(3) & 0.6885(2) & 0.023(1) & 1 \\ \text { C6 } & 0.8331(4) & 0.6992(3) & 0.5867(2) & 0.029(1) & 1 \\ \text { C7 } & 0.7704(4) & 0.8299(3) & 0.6336(2) & 0.033(1) & 1 \\ \text { C14 } & 0.6197(3) & 0.7199(3) & 0.9249(2) & 0.026(1) & 1 \\ \text { C21 } & 0.3592(3) & 0.6528(3) & 0.6772(2) & 0.032(1) & 1\end{array}$




$\begin{array}{llllll}\text { C2 } & 0.7275(3) & 0.4577(3) & 0.6955(2) & 0.023(1) & 1 \\ \text { C28 } & 0.8429(4) & 0.2729(3) & 0.5652(2) & 0.029(1) & 1 \\ \text { C8 } & 0.8549(4) & 0.8209(4) & 0.6822(2) & 0.036(1) & 1 \\ \text { C13 } & 0.6432(3) & 0.8297(3) & 0.9035(2) & 0.028(1) & 1 \\ \text { C4 } & 0.8743(3) & 0.4804(3) & 0.7742(2) & 0.023(1) & 1 \\ \text { C35 } & 1.0364(4) & 0.2845(4) & 0.8954(3) & 0.041(1) & 1 \\ \text { C29 } & 0.8830(3) & 0.2598(3) & 0.7626(2) & 0.025(1) & 1 \\ \text { C3 } & 0.8308(3) & 0.4054(3) & 0.7448(2) & 0.023(1) & 1 \\ \text { C27 } & 0.8483(4) & 0.1725(3) & 0.5177(2) & 0.034(1) & 1 \\ \text { C11 } & 0.4650(3) & 0.8568(3) & 0.8563(2) & 0.029(1) & 1 \\ \text { C34 } & 1.0100(4) & 0.1498(3) & 0.7031(2) & 0.033(1) & 1 \\ \text { C26 } & 0.7404(4) & 0.1427(3) & 0.5512(2) & 0.036(1) & 1 \\ \text { C18 } & 0.7346(4) & 0.8645(4) & 0.9317(2) & 0.037(1) & 1 \\ \text { C12 } & 0.5474(4) & 0.9127(3) & 0.8607(2) & 0.028(1) & 1 \\ \text { C25 } & 0.6262(4) & 0.2160(4) & 0.6318(3) & 0.037(1) & 1 \\ \text { C24 } & 0.6210(3) & 0.3169(3) & 0.6795(2) & 0.029(1) & 1 \\ \text { C30 } & 0.8010(4) & 0.2384(4) & 0.8399(2) & 0.034(1) & 1 \\ \text { C22 } & 0.6253(4) & 0.5483(4) & 0.5036(2) & 0.032(1) & 1 \\ \text { C9 } & 0.9668(4) & 0.6862(4) & 0.6664(2) & 0.035(1) & 1 \\ \text { C15 } & 0.4474(4) & 0.6470(4) & 0.9077(2) & 0.036(1) & 1 \\ \text { C20 } & 0.4872(4) & 0.8264(3) & 0.5960(2) & 0.036(1) & 1 \\ \text { C19 } & 0.6773(4) & 0.6186(4) & 0.9833(2) & 0.035(1) & 1 \\ \text { C5 } & 0.9518(4) & 0.6121(4) & 0.6079(2) & 0.033(1) & 1 \\ \text { C17 } & 0.5224(4) & 1.0494(3) & 0.8377(2) & 0.040(1) & 1 \\ \text { C31 } & 0.8475(4) & 0.1096(4) & 0.8621(3) & 0.040(1) & 1 \\ \text { C33 } & 1.0559(4) & 0.0219(4) & 0.7245(3) & 0.041(1) & 1 \\ \text { C32 } & 0.9769(4) & 0.0009(4) & 0.8043(3) & 0.040(1) & 1 \\ \text { C16 } & 0.3347(4) & 0.9223(4) & 0.8311(3) & 0.041(1) & 1 \\ \text { C37 } & 1.0566(5) & 0.5294(4) & 0.8598(3) & 0.049(1) & 1 \\ \text { C36 } & 1.2045(4) & 0.3046(4) & 0.7063(3) & 0.050(1) & 1\end{array}$

Table S4. Bond lengths ( $\mathrm{A}$ ) for $\mathbf{1}$.

$\begin{array}{lllllllll}\text { atom } & \text { atom } & \text { distance } & \text { atom } & \text { atom } & \text { distance } & \text { atom } & \text { atom } & \text { distance } \\ \text { Zr } & \text { C1 } & 2.254(3) & \mathrm{Zr} & \mathrm{C} 4 & 2.267(3) & \mathrm{Zr} & \mathrm{C} 7 & 2.504(3) \\ \mathrm{Zr} & \mathrm{C} 6 & 2.518(3) & \mathrm{Zr} & \mathrm{C} 13 & 2.534(3) & \mathrm{Zr} & \mathrm{C} 5 & 2.533(3) \\ \mathrm{Zr} & \mathrm{C} 8 & 2.535(3) & \mathrm{Zr} & \mathrm{C} 12 & 2.536(3) & \mathrm{Zr} & \mathrm{C} 9 & 2.554(3) \\ \mathrm{Zr} & \mathrm{C} 10 & 2.558(3) & \mathrm{Zr} & \mathrm{C} 14 & 2.560(3) & \mathrm{Zr} & \mathrm{C} 11 & 2.579(3) \\ \mathrm{Si} 2 & \mathrm{C} 37 & 1.875(4) & \mathrm{Si} 2 & \mathrm{C} 35 & 1.882(4) & \mathrm{Si} 2 & \mathrm{C} 36 & 1.885(4) \\ \mathrm{Si} 2 & \mathrm{C} 4 & 1.887(3) & \mathrm{Si} 1 & \mathrm{C} 22 & 1.874(3) & \mathrm{Si} 1 & \mathrm{C} 21 & 1.882(3) \\ \mathrm{Si} 1 & \mathrm{C} 1 & 1.886(3) & \mathrm{Si} 1 & \mathrm{C} 20 & 1.886(3) & \mathrm{C} 10 & \mathrm{C} 14 & 1.416(5) \\ \mathrm{C} 10 & \mathrm{C} 11 & 1.432(5) & \mathrm{C} 10 & \mathrm{C} 15 & 1.505(5) & \mathrm{C} 23 & \mathrm{C} 24 & 1.386(5) \\ \mathrm{C} 23 & \mathrm{C} 28 & 1.395(5) & \mathrm{C} 23 & \mathrm{C} 2 & 1.505(4) & \mathrm{C} 1 & \mathrm{C} 2 & 1.369(4) \\ \mathrm{C} 6 & \mathrm{C} 5 & 1.387(5) & \mathrm{C} 6 & \mathrm{C} 7 & 1.414(5) & \mathrm{C} 6 & \mathrm{H} 6 \mathrm{~A} & 0.9800 \\ \mathrm{C} 7 & \mathrm{C} 8 & 1.408(5) & \mathrm{C} 7 & \mathrm{H} 7 \mathrm{~A} & 0.9800 & \mathrm{C} 14 & \mathrm{C} 13 & 1.430(5) \\ \mathrm{C} 14 & \mathrm{C} 19 & 1.502(5) & \mathrm{C} 21 & \mathrm{H} 21 \mathrm{~A} & 0.9600 & \mathrm{C} 21 & \mathrm{H} 21 \mathrm{~B} & 0.9600 \\ \mathrm{C} 21 & \mathrm{H} 21 \mathrm{C} & 0.9600 & \mathrm{C} 2 & \mathrm{C} 3 & 1.517(4) & \mathrm{C} 28 & \mathrm{C} 27 & 1.377(5) \\ \mathrm{C} 28 & \mathrm{H} 28 \mathrm{~A} & 0.9300 & \mathrm{C} 8 & \mathrm{C} 9 & 1.400(5) & \mathrm{C} 8 & \mathrm{H} 8 \mathrm{~A} & 0.9800 \\ \mathrm{C} 13 & \mathrm{C} 12 & 1.424(5) & \mathrm{C} 13 & \mathrm{C} 18 & 1.497(5) & \mathrm{C} 4 & \mathrm{C} 3 & 1.360(4) \\ \mathrm{C} 35 & \mathrm{H} 35 \mathrm{~A} & 0.9600 & \mathrm{C} 35 & \mathrm{H} 35 \mathrm{~B} & 0.9600 & \mathrm{C} 35 & \mathrm{H} 35 \mathrm{C} & 0.9600 \\ \mathrm{C} 29 & \mathrm{C} 30 & 1.370(5) & \mathrm{C} 29 & \mathrm{C} 34 & 1.392(5) & \mathrm{C} 29 & \mathrm{C} 3 & 1.534(4) \\ \mathrm{C} 27 & \mathrm{C} 26 & 1.377(5) & \mathrm{C} 27 & \mathrm{H} 27 \mathrm{~A} & 0.9300 & \mathrm{C} 11 & \mathrm{C} 12 & 1.408(5) \\ \mathrm{C} 11 & \mathrm{C} 16 & 1.500(5) & \mathrm{C} 34 & \mathrm{C} 33 & 1.376(5) & \mathrm{C} 34 & \mathrm{H} 34 \mathrm{~A} & 0.9300 \\ \mathrm{C} 26 & \mathrm{C} 25 & 1.381(5) & \mathrm{C} 26 & \mathrm{H} 26 \mathrm{~A} & 0.9300 & \mathrm{C} 18 & \mathrm{H} 18 \mathrm{~A} & 0.9600\end{array}$




$\begin{array}{lllclllll}\text { C18 } & \text { H18B } & 0.9600 & \text { C18 } & \text { H18C } & 0.9600 & \text { C12 } & \text { C17 } & 1.513(5) \\ \text { C25 } & \text { C24 } & 1.384(5) & \text { C25 } & \text { H25A } & 0.9300 & \text { C24 } & \text { H24A } & 0.9300 \\ \text { C30 } & \text { C31 } & 1.389(5) & \text { C30 } & \text { H30A } & 0.9300 & \text { C22 } & \text { H22A } & 0.9600 \\ \text { C22 } & \text { H22B } & 0.9600 & \text { C22 } & \text { H22C } & 0.9600 & \text { C9 } & \text { C5 } & 1.401(5) \\ \text { C9 } & \text { H9A } & 0.9800 & \text { C15 } & \text { H15A } & 0.9600 & \text { C15 } & \text { H15B } & 0.9600 \\ \text { C15 } & \text { H15C } & 0.9600 & \text { C20 } & \text { H20A } & 0.9600 & \text { C20 } & \text { H20B } & 0.9600 \\ \text { C20 } & \text { H20C } & 0.9600 & \text { C19 } & \text { H19A } & 0.9600 & \text { C19 } & \text { H19B } & 0.9600 \\ \text { C19 } & \text { H19C } & 0.9600 & \text { C5 } & \text { H5A } & 0.9800 & \text { C17 } & \text { H17A } & 0.9600 \\ \text { C17 } & \text { H17B } & 0.9600 & \text { C17 } & \text { H17C } & 0.9600 & \text { C31 } & \text { C32 } & 1.387(5) \\ \text { C31 } & \text { H31A } & 0.9300 & \text { C33 } & \text { C32 } & 1.380(5) & \text { C33 } & \text { H33A } & 0.9300 \\ \text { C32 } & \text { H32A } & 0.9300 & \text { C16 } & \text { H16A } & 0.9600 & \text { C16 } & \text { H16B } & 0.9600 \\ \text { C16 } & \text { H16C } & 0.9600 & \text { C37 } & \text { H37A } & 0.9600 & \text { C37 } & \text { H37B } & 0.9600 \\ \text { C37 } & \text { H37C } & 0.9600 & \text { C36 } & \text { H36A } & 0.9600 & \text { C36 } & \text { H36B } & 0.9600 \\ \text { C36 } & \text { H36C } & 0.9600 & & & & & & \end{array}$

Table S5. Bond angles $\left({ }^{\circ}\right)$ for $\mathbf{1 .}$

\begin{tabular}{|c|c|c|c|c|c|c|c|}
\hline atom & atom & atom & angle & atom & atom & atom & angle \\
\hline $\mathrm{C} 1$ & $\mathrm{Zr}$ & $\mathrm{C} 4$ & $84.32(11)$ & $\mathrm{C} 1$ & $\mathrm{Zr}$ & C7 & $98.90(11)$ \\
\hline C4 & $\mathrm{Zr}$ & C7 & $133.19(11)$ & $\mathrm{C} 1$ & $\mathrm{Zr}$ & C6 & $76.28(11)$ \\
\hline $\mathrm{C} 4$ & $\mathrm{Zr}$ & C6 & $107.02(11)$ & $\mathrm{C} 7$ & $\mathrm{Zr}$ & C6 & $32.71(11)$ \\
\hline $\mathrm{C} 1$ & $\mathrm{Zr}$ & $\mathrm{C} 13$ & $136.64(10)$ & $\mathrm{C} 4$ & $\mathrm{Zr}$ & $\mathrm{C} 13$ & $101.34(11)$ \\
\hline $\mathrm{C} 7$ & $\mathrm{Zr}$ & $\mathrm{C} 13$ & $106.97(11)$ & C6 & $\mathrm{Zr}$ & $\mathrm{C} 13$ & $138.80(11)$ \\
\hline $\mathrm{C} 1$ & $\mathrm{Zr}$ & $\mathrm{C} 5$ & $89.90(11)$ & $\mathrm{C} 4$ & $\mathrm{Zr}$ & C5 & $80.02(11)$ \\
\hline C7 & $\mathrm{Zr}$ & C5 & $53.44(11)$ & C6 & $\mathrm{Zr}$ & $\mathrm{C} 5$ & $31.88(11)$ \\
\hline $\mathrm{C} 13$ & $\mathrm{Zr}$ & C5 & $133.45(11)$ & $\mathrm{C} 1$ & $\mathrm{Zr}$ & C8 & $128.95(11)$ \\
\hline $\mathrm{C} 4$ & $\mathrm{Zr}$ & $\mathrm{C} 8$ & $116.10(12)$ & $\mathrm{C} 7$ & $\mathrm{Zr}$ & C8 & $32.45(12)$ \\
\hline C6 & $\mathrm{Zr}$ & $\mathrm{C} 8$ & $53.52(11)$ & $\mathrm{C} 13$ & $\mathrm{Zr}$ & $\mathrm{C} 8$ & $87.34(11)$ \\
\hline C5 & $\mathrm{Zr}$ & $\mathrm{C} 8$ & $53.03(11)$ & $\mathrm{C} 1$ & $\mathrm{Zr}$ & $\mathrm{C} 12$ & $118.75(11)$ \\
\hline $\mathrm{C} 4$ & $\mathrm{Zr}$ & $\mathrm{C} 12$ & $132.14(11)$ & $\mathrm{C} 7$ & $\mathrm{Zr}$ & $\mathrm{C} 12$ & $87.04(11)$ \\
\hline C6 & $\mathrm{Zr}$ & $\mathrm{C} 12$ & $118.57(11)$ & $\mathrm{C} 13$ & $\mathrm{Zr}$ & $\mathrm{C} 12$ & $32.62(11)$ \\
\hline C5 & $\mathrm{Zr}$ & $\mathrm{C} 12$ & $135.54(11)$ & $\mathrm{C} 8$ & $\mathrm{Zr}$ & $\mathrm{C} 12$ & $82.89(11)$ \\
\hline $\mathrm{C} 1$ & $\mathrm{Zr}$ & C9 & $121.86(11)$ & $\mathrm{C} 4$ & $\mathrm{Zr}$ & C9 & $85.18(11)$ \\
\hline C7 & $\mathrm{Zr}$ & C9 & $53.39(12)$ & C6 & $\mathrm{Zr}$ & C9 & $53.07(11)$ \\
\hline $\mathrm{C} 13$ & $\mathrm{Zr}$ & C9 & $101.50(11)$ & C5 & $\mathrm{Zr}$ & C9 & $31.96(12)$ \\
\hline $\mathrm{C} 8$ & $\mathrm{Zr}$ & C9 & $31.92(12)$ & $\mathrm{C} 12$ & $\mathrm{Zr}$ & C9 & $110.24(11)$ \\
\hline $\mathrm{C} 1$ & $\mathrm{Zr}$ & $\mathrm{C} 10$ & $83.13(10)$ & $\mathrm{C} 4$ & $\mathrm{Zr}$ & $\mathrm{C} 10$ & $93.27(10)$ \\
\hline C7 & $\mathrm{Zr}$ & $\mathrm{C} 10$ & $133.54(11)$ & C6 & $\mathrm{Zr}$ & $\mathrm{C} 10$ & $149.19(11)$ \\
\hline $\mathrm{C} 13$ & $\mathrm{Zr}$ & $\mathrm{C} 10$ & $53.82(10)$ & C5 & $\mathrm{Zr}$ & $\mathrm{C} 10$ & $170.81(11)$ \\
\hline $\mathrm{C} 8$ & $\mathrm{Zr}$ & $\mathrm{C} 10$ & $136.15(11)$ & $\mathrm{C} 12$ & $\mathrm{Zr}$ & $\mathrm{C} 10$ & $53.60(10)$ \\
\hline C9 & $\mathrm{Zr}$ & $\mathrm{C} 10$ & $154.53(11)$ & $\mathrm{C} 1$ & $\mathrm{Zr}$ & $\mathrm{C} 14$ & $110.41(10)$ \\
\hline $\mathrm{C} 4$ & $\mathrm{Zr}$ & $\mathrm{C} 14$ & $79.66(10)$ & $\mathrm{C} 7$ & $\mathrm{Zr}$ & $\mathrm{C} 14$ & $138.67(11)$ \\
\hline C6 & $\mathrm{Zr}$ & $\mathrm{C} 14$ & $171.30(10)$ & $\mathrm{C} 13$ & $\mathrm{Zr}$ & $\mathrm{C} 14$ & $32.61(10)$ \\
\hline C5 & $\mathrm{Zr}$ & $\mathrm{C} 14$ & $149.49(11)$ & $\mathrm{C} 8$ & $\mathrm{Zr}$ & $\mathrm{C} 14$ & $118.79(11)$ \\
\hline $\mathrm{C} 12$ & $\mathrm{Zr}$ & $\mathrm{C} 14$ & $53.66(10)$ & C9 & $\mathrm{Zr}$ & $\mathrm{C} 14$ & $123.45(11)$ \\
\hline $\mathrm{C} 10$ & $\mathrm{Zr}$ & $\mathrm{C} 14$ & $32.12(11)$ & $\mathrm{C} 1$ & $\mathrm{Zr}$ & $\mathrm{C} 11$ & $88.13(11)$ \\
\hline $\mathrm{C} 4$ & $\mathrm{Zr}$ & $\mathrm{C} 11$ & $125.64(11)$ & $\mathrm{C} 7$ & $\mathrm{Zr}$ & $\mathrm{C} 11$ & $101.17(11)$ \\
\hline C6 & $\mathrm{Zr}$ & $\mathrm{C} 11$ & $123.14(11)$ & $\mathrm{C} 13$ & $\mathrm{Zr}$ & $\mathrm{C} 11$ & $53.47(11)$ \\
\hline $\mathrm{C} 5$ & $\mathrm{Zr}$ & $\mathrm{C} 11$ & $153.85(11)$ & $\mathrm{C} 8$ & $\mathrm{Zr}$ & $\mathrm{C} 11$ & $110.19(11)$ \\
\hline C12 & $\mathrm{Zr}$ & $\mathrm{C} 11$ & $31.95(11)$ & C9 & $\mathrm{Zr}$ & $\mathrm{C} 11$ & $140.79(11)$ \\
\hline $\mathrm{C} 10$ & $\mathrm{Zr}$ & $\mathrm{C} 11$ & $32.38(10)$ & $\mathrm{C} 14$ & $\mathrm{Zr}$ & $\mathrm{C} 11$ & $53.22(11)$ \\
\hline C37 & $\mathrm{Si} 2$ & C35 & $104.66(18)$ & C37 & $\mathrm{Si} 2$ & C36 & $104.9(2)$ \\
\hline C35 & $\mathrm{Si} 2$ & C36 & $107.83(18)$ & C37 & $\mathrm{Si} 2$ & $\mathrm{C} 4$ & $113.07(17)$ \\
\hline C35 & $\mathrm{Si} 2$ & $\mathrm{C} 4$ & $117.11(16)$ & C36 & $\mathrm{Si} 2$ & $\mathrm{C} 4$ & $108.50(17)$ \\
\hline
\end{tabular}




\begin{tabular}{|c|c|c|c|c|c|c|c|}
\hline $\mathrm{C} 22$ & Sil & $\mathrm{C} 21$ & $107.84(16)$ & $\mathrm{C} 22$ & Sil & $\mathrm{C} 1$ & $113.83(14)$ \\
\hline $\mathrm{C} 21$ & Sil & $\mathrm{C} 1$ & $112.21(15)$ & $\mathrm{C} 22$ & Sil & $\mathrm{C} 20$ & $103.51(16)$ \\
\hline $\mathrm{C} 21$ & Si1 & $\mathrm{C} 20$ & $108.06(16)$ & $\mathrm{C} 1$ & $\mathrm{Si} 1$ & $\mathrm{C} 20$ & $110.89(15)$ \\
\hline C14 & $\mathrm{C} 10$ & $\mathrm{C} 11$ & $107.8(3)$ & C14 & $\mathrm{C} 10$ & $\mathrm{C} 15$ & $126.2(3)$ \\
\hline C11 & $\mathrm{C} 10$ & C15 & $125.6(3)$ & $\mathrm{C} 14$ & $\mathrm{C} 10$ & $\mathrm{Zr}$ & $74.00(17)$ \\
\hline $\mathrm{C} 11$ & $\mathrm{C} 10$ & $\mathrm{Zr}$ & 74.61(17) & $\mathrm{C} 15$ & $\mathrm{C} 10$ & $\mathrm{Zr}$ & $122.9(2)$ \\
\hline C24 & $\mathrm{C} 23$ & $\mathrm{C} 28$ & $117.4(3)$ & $\mathrm{C} 24$ & $\mathrm{C} 23$ & $\mathrm{C} 2$ & $123.0(3)$ \\
\hline C28 & $\mathrm{C} 23$ & $\mathrm{C} 2$ & $119.6(3)$ & $\mathrm{C} 2$ & $\mathrm{C} 1$ & Si1 & $122.1(2)$ \\
\hline C2 & $\mathrm{C} 1$ & $\mathrm{Zr}$ & $104.2(2)$ & Sil & $\mathrm{C} 1$ & $\mathrm{Zr}$ & $132.80(16)$ \\
\hline C5 & C6 & C7 & 107.9(3) & C5 & C6 & $\mathrm{Zr}$ & 74.67(19) \\
\hline C7 & C6 & $\mathrm{Zr}$ & $73.10(18)$ & $\mathrm{C} 5$ & C6 & H6A & 125.7 \\
\hline C7 & C6 & H6A & 125.7 & $\mathrm{Zr}$ & C6 & H6A & 125.7 \\
\hline C6 & C7 & C8 & 107.4(3) & C6 & C7 & $\mathrm{Zr}$ & $74.19(18)$ \\
\hline $\mathrm{C} 8$ & C7 & $\mathrm{Zr}$ & $75.02(19)$ & C6 & $\mathrm{C} 7$ & H7A & 125.8 \\
\hline $\mathrm{C} 8$ & C7 & H7A & 125.8 & $\mathrm{Zr}$ & $\mathrm{C} 7$ & H7A & 125.8 \\
\hline C10 & C14 & C13 & 108.1(3) & $\mathrm{C} 10$ & C14 & C19 & $124.1(3)$ \\
\hline $\mathrm{C} 13$ & C14 & C19 & 127.1(3) & $\mathrm{C} 10$ & C14 & $\mathrm{Zr}$ & $73.88(17)$ \\
\hline $\mathrm{C} 13$ & C14 & $\mathrm{Zr}$ & 72.71(17) & C19 & C14 & $\mathrm{Zr}$ & $126.9(2)$ \\
\hline Si1 & $\mathrm{C} 21$ & $\mathrm{H} 21 \mathrm{~A}$ & 109.5 & Si1 & $\mathrm{C} 21$ & H21B & 109.5 \\
\hline $\mathrm{H} 21 \mathrm{~A}$ & $\mathrm{C} 21$ & H21B & 109.5 & Si1 & $\mathrm{C} 21$ & $\mathrm{H} 21 \mathrm{C}$ & 109.5 \\
\hline $\mathrm{H} 21 \mathrm{~A}$ & $\mathrm{C} 21$ & $\mathrm{H} 21 \mathrm{C}$ & 109.5 & $\mathrm{H} 21 \mathrm{~B}$ & $\mathrm{C} 21$ & $\mathrm{H} 21 \mathrm{C}$ & 109.5 \\
\hline $\mathrm{C} 1$ & $\mathrm{C} 2$ & $\mathrm{C} 23$ & $122.8(3)$ & $\mathrm{C} 1$ & $\mathrm{C} 2$ & C3 & $123.3(3)$ \\
\hline $\mathrm{C} 23$ & $\mathrm{C} 2$ & $\mathrm{C} 3$ & $113.7(3)$ & $\mathrm{C} 27$ & $\mathrm{C} 28$ & $\mathrm{C} 23$ & $121.8(3)$ \\
\hline C27 & $\mathrm{C} 28$ & $\mathrm{H} 28 \mathrm{~A}$ & 119.1 & $\mathrm{C} 23$ & $\mathrm{C} 28$ & $\mathrm{H} 28 \mathrm{~A}$ & 119.1 \\
\hline C9 & C8 & C7 & 108.1(3) & C9 & C8 & $\mathrm{Zr}$ & $74.78(19)$ \\
\hline C7 & $\mathrm{C} 8$ & $\mathrm{Zr}$ & $72.53(19)$ & C9 & $\mathrm{C} 8$ & H8A & 125.7 \\
\hline C7 & $\mathrm{C} 8$ & H8A & 125.7 & $\mathrm{Zr}$ & $\mathrm{C} 8$ & H8A & 125.7 \\
\hline C14 & $\mathrm{C} 13$ & C12 & 107.4(3) & $\mathrm{C} 14$ & $\mathrm{C} 13$ & C18 & $127.3(3)$ \\
\hline C12 & $\mathrm{C} 13$ & C18 & $124.8(3)$ & C14 & $\mathrm{C} 13$ & $\mathrm{Zr}$ & $74.69(17)$ \\
\hline $\mathrm{C} 12$ & $\mathrm{C} 13$ & $\mathrm{Zr}$ & $73.75(18)$ & $\mathrm{C} 18$ & $\mathrm{C} 13$ & $\mathrm{Zr}$ & $123.8(2)$ \\
\hline C3 & $\mathrm{C} 4$ & $\mathrm{Si} 2$ & $122.1(2)$ & C3 & $\mathrm{C} 4$ & $\mathrm{Zr}$ & $103.6(2)$ \\
\hline $\mathrm{Si} 2$ & $\mathrm{C} 4$ & $\mathrm{Zr}$ & $133.96(16)$ & $\mathrm{Si} 2$ & C35 & $\mathrm{H} 35 \mathrm{~A}$ & 109.5 \\
\hline $\mathrm{Si} 2$ & C35 & H35B & 109.5 & H35A & C35 & H35B & 109.5 \\
\hline $\mathrm{Si} 2$ & C35 & $\mathrm{H} 35 \mathrm{C}$ & 109.5 & H35A & C35 & $\mathrm{H} 35 \mathrm{C}$ & 109.5 \\
\hline H35B & $\mathrm{C} 35$ & $\mathrm{H} 35 \mathrm{C}$ & 109.5 & $\mathrm{C} 30$ & $\mathrm{C} 29$ & C34 & 119.3(3) \\
\hline C30 & C29 & C3 & $118.4(3)$ & C34 & C29 & C3 & $122.3(3)$ \\
\hline $\mathrm{C} 4$ & $\mathrm{C} 3$ & $\mathrm{C} 2$ & $123.9(3)$ & $\mathrm{C} 4$ & $\mathrm{C} 3$ & $\mathrm{C} 29$ & $120.6(3)$ \\
\hline $\mathrm{C} 2$ & $\mathrm{C} 3$ & $\mathrm{C} 29$ & $115.5(3)$ & $\mathrm{C} 28$ & $\mathrm{C} 27$ & $\mathrm{C} 26$ & $120.0(3)$ \\
\hline $\mathrm{C} 28$ & $\mathrm{C} 27$ & $\mathrm{H} 27 \mathrm{~A}$ & 120.0 & $\mathrm{C} 26$ & $\mathrm{C} 27$ & $\mathrm{H} 27 \mathrm{~A}$ & 120.0 \\
\hline C12 & C11 & C10 & $107.9(3)$ & $\mathrm{C} 12$ & C11 & C16 & $126.7(3)$ \\
\hline $\mathrm{C} 10$ & C11 & $\mathrm{C} 16$ & $124.7(3)$ & $\mathrm{C} 12$ & $\mathrm{C} 11$ & $\mathrm{Zr}$ & $72.33(18)$ \\
\hline C10 & C11 & $\mathrm{Zr}$ & $73.02(17)$ & $\mathrm{C} 16$ & $\mathrm{C} 11$ & $\mathrm{Zr}$ & $127.6(2)$ \\
\hline C33 & C34 & C29 & $120.0(3)$ & $\mathrm{C} 33$ & C34 & H34A & 120.0 \\
\hline C29 & C34 & H34A & 120.0 & $\mathrm{C} 27$ & $\mathrm{C} 26$ & $\mathrm{C} 25$ & $119.2(3)$ \\
\hline $\mathrm{C} 27$ & $\mathrm{C} 26$ & H26A & 120.4 & $\mathrm{C} 25$ & $\mathrm{C} 26$ & H26A & 120.4 \\
\hline C13 & C18 & H18A & 109.5 & C13 & $\mathrm{C} 18$ & H18B & 109.5 \\
\hline H18A & C18 & H18B & 109.5 & C13 & $\mathrm{C} 18$ & H18C & 109.5 \\
\hline H18A & $\mathrm{C} 18$ & $\mathrm{H} 18 \mathrm{C}$ & 109.5 & H18B & $\mathrm{C} 18$ & $\mathrm{H} 18 \mathrm{C}$ & 109.5 \\
\hline C11 & $\mathrm{C} 12$ & C13 & $108.6(3)$ & $\mathrm{C} 11$ & $\mathrm{C} 12$ & $\mathrm{C} 17$ & $125.7(3)$ \\
\hline C13 & $\mathrm{C} 12$ & $\mathrm{C} 17$ & $125.0(3)$ & $\mathrm{C} 11$ & $\mathrm{C} 12$ & $\mathrm{Zr}$ & $75.72(18)$ \\
\hline C13 & $\mathrm{C} 12$ & $\mathrm{Zr}$ & $73.63(18)$ & $\mathrm{C} 17$ & $\mathrm{C} 12$ & $\mathrm{Zr}$ & $124.2(2)$ \\
\hline $\mathrm{C} 26$ & $\mathrm{C} 25$ & $\mathrm{C} 24$ & $120.6(3)$ & $\mathrm{C} 26$ & $\mathrm{C} 25$ & $\mathrm{H} 25 \mathrm{~A}$ & 119.7 \\
\hline $\mathrm{C} 24$ & $\mathrm{C} 25$ & $\mathrm{H} 25 \mathrm{~A}$ & 119.7 & $\mathrm{C} 25$ & $\mathrm{C} 24$ & $\mathrm{C} 23$ & $120.9(3)$ \\
\hline $\mathrm{C} 25$ & $\mathrm{C} 24$ & $\mathrm{H} 24 \mathrm{~A}$ & 119.5 & $\mathrm{C} 23$ & $\mathrm{C} 24$ & $\mathrm{H} 24 \mathrm{~A}$ & 119.5 \\
\hline C29 & $\mathrm{C} 30$ & C31 & $120.8(3)$ & C29 & C30 & $\mathrm{H} 30 \mathrm{~A}$ & 119.6 \\
\hline C31 & C30 & $\mathrm{H} 30 \mathrm{~A}$ & 119.6 & Si1 & $\mathrm{C} 22$ & $\mathrm{H} 22 \mathrm{~A}$ & 109.5 \\
\hline
\end{tabular}




\begin{tabular}{|c|c|c|c|c|c|c|c|}
\hline $\mathrm{Si} 1$ & $\mathrm{C} 22$ & H22B & 109.5 & $\mathrm{H} 22 \mathrm{~A}$ & $\mathrm{C} 22$ & H22B & 109.5 \\
\hline Si1 & $\mathrm{C} 22$ & $\mathrm{H} 22 \mathrm{C}$ & 109.5 & $\mathrm{H} 22 \mathrm{~A}$ & C22 & $\mathrm{H} 22 \mathrm{C}$ & 109.5 \\
\hline H22B & $\mathrm{C} 22$ & $\mathrm{H} 22 \mathrm{C}$ & 109.5 & C8 & C9 & C5 & $107.8(3)$ \\
\hline C8 & C9 & $\mathrm{Zr}$ & $73.30(19)$ & $\mathrm{C} 5$ & C9 & $\mathrm{Zr}$ & $73.18(19)$ \\
\hline C8 & C9 & H9A & 125.8 & C5 & C9 & H9A & 125.8 \\
\hline $\mathrm{Zr}$ & C9 & H9A & 125.8 & $\mathrm{C} 10$ & C15 & H15A & 109.5 \\
\hline $\mathrm{C} 10$ & C15 & H15B & 109.5 & H15A & C15 & H15B & 109.5 \\
\hline $\mathrm{C} 10$ & $\mathrm{C} 15$ & H15C & 109.5 & H15A & C15 & $\mathrm{H} 15 \mathrm{C}$ & 109.5 \\
\hline H15B & $\mathrm{C} 15$ & H15C & 109.5 & Si1 & $\mathrm{C} 20$ & $\mathrm{H} 20 \mathrm{~A}$ & 109.5 \\
\hline Si1 & $\mathrm{C} 20$ & H20B & 109.5 & $\mathrm{H} 20 \mathrm{~A}$ & $\mathrm{C} 20$ & H20B & 109.5 \\
\hline Sil & $\mathrm{C} 20$ & $\mathrm{H} 20 \mathrm{C}$ & 109.5 & $\mathrm{H} 20 \mathrm{~A}$ & $\mathrm{C} 20$ & $\mathrm{H} 20 \mathrm{C}$ & 109.5 \\
\hline H20B & $\mathrm{C} 20$ & H20C & 109.5 & C14 & C19 & H19A & 109.5 \\
\hline C14 & C19 & H19B & 109.5 & H19A & C19 & H19B & 109.5 \\
\hline C14 & C19 & H19C & 109.5 & H19A & C19 & H19C & 109.5 \\
\hline H19B & C19 & H19C & 109.5 & C6 & C5 & C9 & $108.8(3)$ \\
\hline C6 & C5 & $\mathrm{Zr}$ & $73.45(18)$ & C9 & $\mathrm{C} 5$ & $\mathrm{Zr}$ & $74.86(19)$ \\
\hline C6 & $\mathrm{C} 5$ & H5A & 125.3 & $\mathrm{C} 9$ & $\mathrm{C} 5$ & H5A & 125.3 \\
\hline $\mathrm{Zr}$ & $\mathrm{C} 5$ & H5A & 125.3 & C12 & C17 & H17A & 109.5 \\
\hline $\mathrm{C} 12$ & C17 & H17B & 109.5 & H17A & C17 & H17B & 109.5 \\
\hline $\mathrm{C} 12$ & $\mathrm{C} 17$ & H17C & 109.5 & H17A & $\mathrm{C} 17$ & H17C & 109.5 \\
\hline H17B & $\mathrm{C} 17$ & H17C & 109.5 & C32 & C 31 & C30 & $119.6(3)$ \\
\hline C32 & C31 & $\mathrm{H} 31 \mathrm{~A}$ & 120.2 & C30 & C31 & $\mathrm{H} 31 \mathrm{~A}$ & 120.2 \\
\hline C34 & C33 & C32 & $120.7(3)$ & C34 & C33 & H33A & 119.6 \\
\hline C32 & $\mathrm{C} 33$ & H33A & 119.6 & C33 & C 32 & C31 & 119.4(3) \\
\hline C33 & C32 & $\mathrm{H} 32 \mathrm{~A}$ & 120.3 & C31 & C32 & H32A & 120.3 \\
\hline $\mathrm{C} 11$ & $\mathrm{C} 16$ & H16A & 109.5 & $\mathrm{C} 11$ & $\mathrm{C} 16$ & H16B & 109.5 \\
\hline H16A & $\mathrm{C} 16$ & H16B & 109.5 & $\mathrm{C} 11$ & C16 & $\mathrm{H} 16 \mathrm{C}$ & 109.5 \\
\hline H16A & $\mathrm{C} 16$ & $\mathrm{H} 16 \mathrm{C}$ & 109.5 & H16B & C16 & H16C & 109.5 \\
\hline $\mathrm{Si} 2$ & C37 & H37A & 109.5 & $\mathrm{Si} 2$ & C37 & H37B & 109.5 \\
\hline H37A & C37 & H37B & 109.5 & $\mathrm{Si} 2$ & C37 & $\mathrm{H} 37 \mathrm{C}$ & 109.5 \\
\hline H37A & C37 & H37C & 109.5 & H37B & C37 & H37C & 109.5 \\
\hline $\mathrm{Si} 2$ & C36 & H36A & 109.5 & $\mathrm{Si} 2$ & C36 & H36B & 109.5 \\
\hline H36A & C36 & H36B & 109.5 & $\mathrm{Si} 2$ & C36 & H36C & 109.5 \\
\hline H36A & C36 & $\mathrm{H} 36 \mathrm{C}$ & 109.5 & H36B & C36 & $\mathrm{H} 36 \mathrm{C}$ & 109.5 \\
\hline
\end{tabular}

Table S6. Torsion angles $\left(^{\circ}\right)$ for $\mathbf{1}$.

$\begin{array}{llllllllll}\text { atom } & \text { atom } & \text { atom } & \text { atom } & \text { angle } & \text { atom } & \text { atom } & \text { atom } & \text { atom } & \text { angle } \\ \text { C1 } & \mathrm{Zr} & \mathrm{C} 10 & \mathrm{C} 14 & 148.5(2) & \mathrm{C} 4 & \mathrm{Zr} & \mathrm{C} 10 & \mathrm{C} 14 & 64.6(2) \\ \text { C7 } & \mathrm{Zr} & \mathrm{C} 10 & \mathrm{C} 14 & -115.8(2) & \mathrm{C} 6 & \mathrm{Zr} & \mathrm{C} 10 & \mathrm{C} 14 & -163.5(2) \\ \text { C13 } & \mathrm{Zr} & \mathrm{C} 10 & \mathrm{C} 14 & -37.08(18) & \mathrm{C} 5 & \mathrm{Zr} & \mathrm{C} 10 & \mathrm{C} 14 & 107.5(7) \\ \text { C8 } & \mathrm{Zr} & \mathrm{C} 10 & \mathrm{C} 14 & -69.5(2) & \mathrm{C} 12 & \mathrm{Zr} & \mathrm{C} 10 & \mathrm{C} 14 & -77.9(2) \\ \text { C9 } & \mathrm{Zr} & \mathrm{C} 10 & \mathrm{C} 14 & -21.1(4) & \mathrm{C} 11 & \mathrm{Zr} & \mathrm{C} 10 & \mathrm{C} 14 & -114.2(3) \\ \text { C1 } & \mathrm{Zr} & \mathrm{C} 10 & \mathrm{C} 11 & -97.4(2) & \mathrm{C} 4 & \mathrm{Zr} & \mathrm{C} 10 & \mathrm{C} 11 & 178.7(2) \\ \text { C7 } & \mathrm{Zr} & \mathrm{C} 10 & \mathrm{C} 11 & -1.6(3) & \mathrm{C} 6 & \mathrm{Zr} & \mathrm{C} 10 & \mathrm{C} 11 & -49.3(3) \\ \text { C13 } & \mathrm{Zr} & \mathrm{C} 10 & \mathrm{C} 11 & 77.1(2) & \mathrm{C} 5 & \mathrm{Zr} & \mathrm{C} 10 & \mathrm{C} 11 & -138.4(6) \\ \text { C8 } & \mathrm{Zr} & \mathrm{C} 10 & \mathrm{C} 11 & 44.7(3) & \mathrm{C} 12 & \mathrm{Zr} & \mathrm{C} 10 & \mathrm{C} 11 & 36.30(19) \\ \text { C9 } & \mathrm{Zr} & \mathrm{C} 10 & \mathrm{C} 11 & 93.1(3) & \mathrm{C} 14 & \mathrm{Zr} & \mathrm{C} 10 & \mathrm{C} 11 & 114.2(3) \\ \text { C1 } & \mathrm{Zr} & \mathrm{C} 10 & \mathrm{C} 15 & 25.4(3) & \mathrm{C} 4 & \mathrm{Zr} & \mathrm{C} 10 & \mathrm{C} 15 & -58.5(3) \\ \text { C7 } & \mathrm{Zr} & \mathrm{C} 10 & \mathrm{C} 15 & 121.1(3) & \mathrm{C} 6 & \mathrm{Zr} & \mathrm{C} 10 & \mathrm{C} 15 & 73.4(3) \\ \text { C13 } & \mathrm{Zr} & \mathrm{C} 10 & \mathrm{C} 15 & -160.2(3) & \mathrm{C} 5 & \mathrm{Zr} & \mathrm{C} 10 & \mathrm{C} 15 & -15.6(8) \\ \text { C8 } & \mathrm{Zr} & \mathrm{C} 10 & \mathrm{C} 15 & 167.4(3) & \mathrm{C} 12 & \mathrm{Zr} & \mathrm{C} 10 & \mathrm{C} 15 & 159.1(3)\end{array}$




\begin{tabular}{|c|c|c|c|c|c|c|c|c|c|}
\hline C9 & $\mathrm{Zr}$ & $\mathrm{C} 10$ & $\mathrm{C} 15$ & $-144.2(3)$ & C14 & $\mathrm{Zr}$ & $\mathrm{C} 10$ & C15 & $-123.1(4)$ \\
\hline $\mathrm{C} 11$ & $\mathrm{Zr}$ & $\mathrm{C} 10$ & C15 & $122.8(4)$ & $\mathrm{C} 22$ & Si1 & $\mathrm{C} 1$ & $\mathrm{C} 2$ & $-47.1(3)$ \\
\hline $\mathrm{C} 21$ & Si1 & $\mathrm{C} 1$ & $\mathrm{C} 2$ & 75.7(3) & $\mathrm{C} 20$ & Sil & $\mathrm{C} 1$ & $\mathrm{C} 2$ & $-163.4(3)$ \\
\hline $\mathrm{C} 22$ & Si1 & $\mathrm{C} 1$ & $\mathrm{Zr}$ & $120.1(2)$ & $\mathrm{C} 21$ & Si1 & $\mathrm{C} 1$ & $\mathrm{Zr}$ & $-117.1(2)$ \\
\hline $\mathrm{C} 20$ & Sil & $\mathrm{C} 1$ & $\mathrm{Zr}$ & $3.9(3)$ & $\mathrm{C} 4$ & $\mathrm{Zr}$ & $\mathrm{C} 1$ & $\mathrm{C} 2$ & $0.0(2)$ \\
\hline C7 & $\mathrm{Zr}$ & $\mathrm{C} 1$ & $\mathrm{C} 2$ & $132.9(2)$ & C6 & $\mathrm{Zr}$ & $\mathrm{C} 1$ & $\mathrm{C} 2$ & 109.1(2) \\
\hline C13 & $\mathrm{Zr}$ & $\mathrm{C} 1$ & $\mathrm{C} 2$ & $-100.5(2)$ & C5 & $\mathrm{Zr}$ & $\mathrm{C} 1$ & $\mathrm{C} 2$ & $80.0(2)$ \\
\hline $\mathrm{C} 8$ & $\mathrm{Zr}$ & $\mathrm{C} 1$ & $\mathrm{C} 2$ & $119.2(2)$ & $\mathrm{C} 12$ & $\mathrm{Zr}$ & $\mathrm{C} 1$ & $\mathrm{C} 2$ & $-135.64(19)$ \\
\hline C9 & $\mathrm{Zr}$ & $\mathrm{C} 1$ & $\mathrm{C} 2$ & $80.7(2)$ & $\mathrm{C} 10$ & $\mathrm{Zr}$ & $\mathrm{C} 1$ & $\mathrm{C} 2$ & $-94.0(2)$ \\
\hline C14 & $\mathrm{Zr}$ & $\mathrm{C} 1$ & $\mathrm{C} 2$ & $-76.8(2)$ & C11 & $\mathrm{Zr}$ & $\mathrm{C} 1$ & $\mathrm{C} 2$ & $-126.1(2)$ \\
\hline $\mathrm{C} 4$ & $\mathrm{Zr}$ & $\mathrm{C} 1$ & Si1 & $-168.9(2)$ & $\mathrm{C} 7$ & $\mathrm{Zr}$ & $\mathrm{C} 1$ & Sil & $-36.0(2)$ \\
\hline C6 & $\mathrm{Zr}$ & $\mathrm{C} 1$ & Si1 & $-59.8(2)$ & $\mathrm{C} 13$ & $\mathrm{Zr}$ & $\mathrm{C} 1$ & Sil & $90.6(2)$ \\
\hline C5 & $\mathrm{Zr}$ & $\mathrm{C} 1$ & Si1 & $-88.9(2)$ & $\mathrm{C} 8$ & $\mathrm{Zr}$ & $\mathrm{C} 1$ & Sil & $-49.7(3)$ \\
\hline C12 & $\mathrm{Zr}$ & $\mathrm{C} 1$ & Si1 & $55.5(2)$ & C9 & $\mathrm{Zr}$ & $\mathrm{C} 1$ & Sil & $-88.2(2)$ \\
\hline C10 & $\mathrm{Zr}$ & $\mathrm{C} 1$ & Si1 & $97.1(2)$ & C14 & $\mathrm{Zr}$ & $\mathrm{C} 1$ & Sil & $114.38(19)$ \\
\hline C11 & $\mathrm{Zr}$ & $\mathrm{C} 1$ & Si1 & $65.0(2)$ & $\mathrm{C} 1$ & $\mathrm{Zr}$ & C6 & C5 & $-112.9(2)$ \\
\hline $\mathrm{C} 4$ & $\mathrm{Zr}$ & C6 & C5 & $-33.4(2)$ & C7 & $\mathrm{Zr}$ & C6 & $\mathrm{C} 5$ & 114.6(3) \\
\hline C13 & $\mathrm{Zr}$ & C6 & C5 & $98.1(2)$ & C8 & $\mathrm{Zr}$ & C6 & $\mathrm{C} 5$ & $76.9(2)$ \\
\hline $\mathrm{C} 12$ & $\mathrm{Zr}$ & C6 & $\mathrm{C} 5$ & $131.6(2)$ & C9 & $\mathrm{Zr}$ & C6 & $\mathrm{C} 5$ & $36.8(2)$ \\
\hline C10 & $\mathrm{Zr}$ & C6 & C5 & $-162.4(2)$ & C14 & $\mathrm{Zr}$ & C6 & $\mathrm{C} 5$ & $106.1(7)$ \\
\hline C11 & $\mathrm{Zr}$ & C6 & C5 & $168.59(19)$ & $\mathrm{C} 1$ & $\mathrm{Zr}$ & C6 & C7 & $132.5(2)$ \\
\hline $\mathrm{C} 4$ & $\mathrm{Zr}$ & C6 & C7 & $-148.0(2)$ & $\mathrm{C} 13$ & $\mathrm{Zr}$ & C6 & C7 & $-16.5(3)$ \\
\hline C5 & $\mathrm{Zr}$ & C6 & $\mathrm{C} 7$ & $-114.6(3)$ & $\mathrm{C} 8$ & $\mathrm{Zr}$ & C6 & $\mathrm{C} 7$ & $-37.7(2)$ \\
\hline C12 & $\mathrm{Zr}$ & C6 & C7 & $16.9(2)$ & C9 & $\mathrm{Zr}$ & C6 & C7 & $-77.9(2)$ \\
\hline $\mathrm{C} 10$ & $\mathrm{Zr}$ & C6 & C7 & $83.0(3)$ & C14 & $\mathrm{Zr}$ & C6 & C7 & $-8.6(8)$ \\
\hline C11 & $\mathrm{Zr}$ & C6 & C7 & $54.0(2)$ & C5 & C6 & $\mathrm{C} 7$ & $\mathrm{C} 8$ & 1.1(4) \\
\hline $\mathrm{Zr}$ & C6 & $\mathrm{C} 7$ & C8 & $68.3(2)$ & $\mathrm{C} 5$ & C6 & $\mathrm{C} 7$ & $\mathrm{Zr}$ & $-67.1(2)$ \\
\hline $\mathrm{C} 1$ & $\mathrm{Zr}$ & C7 & C6 & $-46.5(2)$ & $\mathrm{C} 4$ & $\mathrm{Zr}$ & C7 & C6 & $44.0(3)$ \\
\hline C13 & $\mathrm{Zr}$ & C7 & C6 & $168.69(19)$ & C5 & $\mathrm{Zr}$ & C7 & C6 & $36.7(2)$ \\
\hline C8 & $\mathrm{Zr}$ & C7 & C6 & $113.5(3)$ & $\mathrm{C} 12$ & $\mathrm{Zr}$ & $\mathrm{C} 7$ & C6 & $-165.2(2)$ \\
\hline C9 & $\mathrm{Zr}$ & $\mathrm{C} 7$ & C6 & $76.8(2)$ & $\mathrm{C} 10$ & $\mathrm{Zr}$ & $\mathrm{C} 7$ & C6 & $-135.5(2)$ \\
\hline C14 & $\mathrm{Zr}$ & C7 & C6 & $178.05(18)$ & C11 & $\mathrm{Zr}$ & C7 & C6 & $-136.3(2)$ \\
\hline $\mathrm{C} 1$ & $\mathrm{Zr}$ & $\mathrm{C} 7$ & C8 & $-160.0(2)$ & $\mathrm{C} 4$ & $\mathrm{Zr}$ & $\mathrm{C} 7$ & $\mathrm{C} 8$ & $-69.4(3)$ \\
\hline C6 & $\mathrm{Zr}$ & $\mathrm{C} 7$ & $\mathrm{C} 8$ & $-113.5(3)$ & $\mathrm{C} 13$ & $\mathrm{Zr}$ & $\mathrm{C} 7$ & $\mathrm{C} 8$ & $55.2(2)$ \\
\hline C5 & $\mathrm{Zr}$ & C7 & C8 & $-76.8(2)$ & $\mathrm{C} 12$ & $\mathrm{Zr}$ & $\mathrm{C} 7$ & C8 & $81.4(2)$ \\
\hline C9 & $\mathrm{Zr}$ & C7 & C8 & $-36.7(2)$ & $\mathrm{C} 10$ & $\mathrm{Zr}$ & $\mathrm{C} 7$ & $\mathrm{C} 8$ & 111.1(2) \\
\hline C14 & $\mathrm{Zr}$ & C7 & C8 & $64.6(3)$ & C11 & $\mathrm{Zr}$ & $\mathrm{C} 7$ & C8 & $110.2(2)$ \\
\hline C11 & $\mathrm{C} 10$ & $\mathrm{C} 14$ & $\mathrm{C} 13$ & $-2.4(3)$ & $\mathrm{C} 15$ & $\mathrm{C} 10$ & $\mathrm{C} 14$ & $\mathrm{C} 13$ & $-175.5(3)$ \\
\hline $\mathrm{Zr}$ & $\mathrm{C} 10$ & $\mathrm{C} 14$ & C13 & $65.1(2)$ & C11 & $\mathrm{C} 10$ & $\mathrm{C} 14$ & C19 & $168.5(3)$ \\
\hline C15 & $\mathrm{C} 10$ & $\mathrm{C} 14$ & C19 & $-4.6(5)$ & $\mathrm{Zr}$ & $\mathrm{C} 10$ & C14 & C19 & $-123.9(3)$ \\
\hline C11 & $\mathrm{C} 10$ & $\mathrm{C} 14$ & $\mathrm{Zr}$ & $-67.5(2)$ & $\mathrm{C} 15$ & $\mathrm{C} 10$ & C14 & $\mathrm{Zr}$ & 119.3(3) \\
\hline $\mathrm{C} 1$ & $\mathrm{Zr}$ & $\mathrm{C} 14$ & $\mathrm{C} 10$ & $-33.7(2)$ & $\mathrm{C} 4$ & $\mathrm{Zr}$ & $\mathrm{C} 14$ & $\mathrm{C} 10$ & $-113.6(2)$ \\
\hline C7 & $\mathrm{Zr}$ & $\mathrm{C} 14$ & $\mathrm{C} 10$ & $98.7(2)$ & C6 & $\mathrm{Zr}$ & $\mathrm{C} 14$ & $\mathrm{C} 10$ & $105.7(7)$ \\
\hline C13 & $\mathrm{Zr}$ & $\mathrm{C} 14$ & $\mathrm{C} 10$ & $115.4(3)$ & C5 & $\mathrm{Zr}$ & $\mathrm{C} 14$ & $\mathrm{C} 10$ & $-162.5(2)$ \\
\hline C8 & $\mathrm{Zr}$ & $\mathrm{C} 14$ & $\mathrm{C} 10$ & $132.23(19)$ & $\mathrm{C} 12$ & $\mathrm{Zr}$ & $\mathrm{C} 14$ & $\mathrm{C} 10$ & $77.7(2)$ \\
\hline C9 & $\mathrm{Zr}$ & $\mathrm{C} 14$ & $\mathrm{C} 10$ & $169.32(18)$ & C11 & $\mathrm{Zr}$ & $\mathrm{C} 14$ & $\mathrm{C} 10$ & $37.59(18)$ \\
\hline $\mathrm{C} 1$ & $\mathrm{Zr}$ & $\mathrm{C} 14$ & C13 & $-149.09(19)$ & $\mathrm{C} 4$ & $\mathrm{Zr}$ & C14 & $\mathrm{C} 13$ & $131.0(2)$ \\
\hline C7 & $\mathrm{Zr}$ & $\mathrm{C} 14$ & C13 & $-16.8(3)$ & C6 & $\mathrm{Zr}$ & $\mathrm{C} 14$ & $\mathrm{C} 13$ & $-9.8(8)$ \\
\hline $\mathrm{C} 5$ & $\mathrm{Zr}$ & $\mathrm{C} 14$ & C13 & $82.0(3)$ & $\mathrm{C} 8$ & $\mathrm{Zr}$ & $\mathrm{C} 14$ & $\mathrm{C} 13$ & $16.8(2)$ \\
\hline $\mathrm{C} 12$ & $\mathrm{Zr}$ & $\mathrm{C} 14$ & $\mathrm{C} 13$ & $-37.77(19)$ & $\mathrm{C} 9$ & $\mathrm{Zr}$ & $\mathrm{C} 14$ & $\mathrm{C} 13$ & $53.9(2)$ \\
\hline $\mathrm{C} 10$ & $\mathrm{Zr}$ & $\mathrm{C} 14$ & C13 & $-115.4(3)$ & C11 & $\mathrm{Zr}$ & C14 & $\mathrm{C} 13$ & $-77.8(2)$ \\
\hline C1 & $\mathrm{Zr}$ & $\mathrm{C} 14$ & C19 & 87.1(3) & $\mathrm{C} 4$ & $\mathrm{Zr}$ & $\mathrm{C} 14$ & C19 & $7.2(3)$ \\
\hline C7 & $\mathrm{Zr}$ & $\mathrm{C} 14$ & C19 & $-140.6(3)$ & C6 & $\mathrm{Zr}$ & C14 & C19 & $-133.6(6)$ \\
\hline C13 & $\mathrm{Zr}$ & $\mathrm{C} 14$ & C19 & $-123.8(4)$ & C5 & $\mathrm{Zr}$ & $\mathrm{C} 14$ & C19 & $-41.8(4)$ \\
\hline $\mathrm{C} 8$ & $\mathrm{Zr}$ & $\mathrm{C} 14$ & C19 & $-107.0(3)$ & $\mathrm{C} 12$ & $\mathrm{Zr}$ & C14 & C19 & $-161.6(3)$ \\
\hline
\end{tabular}




\begin{tabular}{|c|c|c|c|c|c|c|c|c|c|}
\hline C9 & $\mathrm{Zr}$ & C14 & C19 & $-69.9(3)$ & $\mathrm{C} 10$ & $\mathrm{Zr}$ & C14 & C19 & $120.8(4)$ \\
\hline C11 & $\mathrm{Zr}$ & $\mathrm{C} 14$ & C19 & $158.4(3)$ & Si1 & $\mathrm{C} 1$ & $\mathrm{C} 2$ & $\mathrm{C} 23$ & $1.6(4)$ \\
\hline $\mathrm{Zr}$ & $\mathrm{C} 1$ & $\mathrm{C} 2$ & $\mathrm{C} 23$ & $-168.8(2)$ & Si1 & $\mathrm{C} 1$ & $\mathrm{C} 2$ & $\mathrm{C} 3$ & $175.2(2)$ \\
\hline $\mathrm{Zr}$ & $\mathrm{C} 1$ & $\mathrm{C} 2$ & C3 & $4.9(3)$ & $\mathrm{C} 24$ & $\mathrm{C} 23$ & $\mathrm{C} 2$ & $\mathrm{C} 1$ & $-82.2(4)$ \\
\hline $\mathrm{C} 28$ & $\mathrm{C} 23$ & $\mathrm{C} 2$ & $\mathrm{C} 1$ & $97.4(4)$ & $\mathrm{C} 24$ & $\mathrm{C} 23$ & $\mathrm{C} 2$ & $\mathrm{C} 3$ & $103.6(3)$ \\
\hline C28 & $\mathrm{C} 23$ & $\mathrm{C} 2$ & $\mathrm{C} 3$ & $-76.8(3)$ & $\mathrm{C} 24$ & $\mathrm{C} 23$ & $\mathrm{C} 28$ & $\mathrm{C} 27$ & $0.5(5)$ \\
\hline C2 & $\mathrm{C} 23$ & C28 & $\mathrm{C} 27$ & $-179.2(3)$ & C6 & C7 & C8 & C9 & $-0.8(4)$ \\
\hline $\mathrm{Zr}$ & C7 & C8 & C9 & $66.9(2)$ & C6 & C7 & $\mathrm{C} 8$ & $\mathrm{Zr}$ & $-67.7(2)$ \\
\hline $\mathrm{C} 1$ & $\mathrm{Zr}$ & C8 & C9 & $-89.2(2)$ & $\mathrm{C} 4$ & $\mathrm{Zr}$ & C8 & C9 & $15.5(2)$ \\
\hline C7 & $\mathrm{Zr}$ & C8 & C9 & $-115.0(3)$ & C6 & $\mathrm{Zr}$ & C8 & C9 & $-76.9(2)$ \\
\hline C13 & $\mathrm{Zr}$ & C8 & C9 & $116.9(2)$ & C5 & $\mathrm{Zr}$ & C8 & C9 & $-36.9(2)$ \\
\hline C12 & $\mathrm{Zr}$ & C8 & C9 & $149.3(2)$ & $\mathrm{C} 10$ & $\mathrm{Zr}$ & $\mathrm{C} 8$ & C9 & $142.5(2)$ \\
\hline $\mathrm{C} 14$ & $\mathrm{Zr}$ & C8 & C9 & $107.9(2)$ & C11 & $\mathrm{Zr}$ & C8 & C9 & $166.2(2)$ \\
\hline $\mathrm{C} 1$ & $\mathrm{Zr}$ & C8 & C7 & $25.8(3)$ & $\mathrm{C} 4$ & $\mathrm{Zr}$ & C8 & C7 & $130.5(2)$ \\
\hline C6 & $\mathrm{Zr}$ & C8 & $\mathrm{C} 7$ & $38.1(2)$ & $\mathrm{C} 13$ & $\mathrm{Zr}$ & $\mathrm{C} 8$ & $\mathrm{C} 7$ & $-128.1(2)$ \\
\hline C5 & $\mathrm{Zr}$ & C8 & C7 & $78.1(2)$ & C12 & $\mathrm{Zr}$ & C8 & C7 & $-95.7(2)$ \\
\hline C9 & $\mathrm{Zr}$ & C8 & C7 & $115.0(3)$ & $\mathrm{C} 10$ & $\mathrm{Zr}$ & C8 & $\mathrm{C} 7$ & $-102.5(2)$ \\
\hline C14 & $\mathrm{Zr}$ & C8 & $\mathrm{C} 7$ & $-137.1(2)$ & C11 & $\mathrm{Zr}$ & C8 & C7 & $-78.8(2)$ \\
\hline $\mathrm{C} 10$ & $\mathrm{C} 14$ & $\mathrm{C} 13$ & $\mathrm{C} 12$ & $1.1(3)$ & C19 & C14 & $\mathrm{C} 13$ & $\mathrm{C} 12$ & $-169.4(3)$ \\
\hline $\mathrm{Zr}$ & $\mathrm{C} 14$ & $\mathrm{C} 13$ & $\mathrm{C} 12$ & 67.1(2) & $\mathrm{C} 10$ & C14 & $\mathrm{C} 13$ & $\mathrm{C} 18$ & $173.0(3)$ \\
\hline C19 & $\mathrm{C} 14$ & $\mathrm{C} 13$ & $\mathrm{C} 18$ & $2.5(5)$ & $\mathrm{Zr}$ & C14 & $\mathrm{C} 13$ & $\mathrm{C} 18$ & $-121.0(3)$ \\
\hline C10 & $\mathrm{C} 14$ & $\mathrm{C} 13$ & $\mathrm{Zr}$ & $-65.9(2)$ & C19 & C14 & $\mathrm{C} 13$ & $\mathrm{Zr}$ & $123.5(3)$ \\
\hline C1 & $\mathrm{Zr}$ & $\mathrm{C} 13$ & $\mathrm{C} 14$ & $44.5(3)$ & $\mathrm{C} 4$ & $\mathrm{Zr}$ & $\mathrm{C} 13$ & $\mathrm{C} 14$ & $-49.2(2)$ \\
\hline C7 & $\mathrm{Zr}$ & $\mathrm{C} 13$ & C14 & 168.51(19) & C6 & $\mathrm{Zr}$ & $\mathrm{C} 13$ & $\mathrm{C} 14$ & 177.77(18) \\
\hline C5 & $\mathrm{Zr}$ & $\mathrm{C} 13$ & $\mathrm{C} 14$ & $-136.2(2)$ & C8 & $\mathrm{Zr}$ & $\mathrm{C} 13$ & $\mathrm{C} 14$ & $-165.3(2)$ \\
\hline C12 & $\mathrm{Zr}$ & $\mathrm{C} 13$ & $\mathrm{C} 14$ & 113.7(3) & C9 & $\mathrm{Zr}$ & $\mathrm{C} 13$ & $\mathrm{C} 14$ & $-136.5(2)$ \\
\hline $\mathrm{C} 10$ & $\mathrm{Zr}$ & $\mathrm{C} 13$ & $\mathrm{C} 14$ & $36.51(18)$ & $\mathrm{C} 11$ & $\mathrm{Zr}$ & $\mathrm{C} 13$ & $\mathrm{C} 14$ & $77.0(2)$ \\
\hline $\mathrm{C} 1$ & $\mathrm{Zr}$ & $\mathrm{C} 13$ & $\mathrm{C} 12$ & $-69.2(2)$ & $\mathrm{C} 4$ & $\mathrm{Zr}$ & $\mathrm{C} 13$ & $\mathrm{C} 12$ & $-162.96(19)$ \\
\hline C7 & $\mathrm{Zr}$ & $\mathrm{C} 13$ & $\mathrm{C} 12$ & $54.8(2)$ & C6 & $\mathrm{Zr}$ & $\mathrm{C} 13$ & $\mathrm{C} 12$ & $64.0(2)$ \\
\hline C5 & $\mathrm{Zr}$ & $\mathrm{C} 13$ & $\mathrm{C} 12$ & $110.1(2)$ & $\mathrm{C} 8$ & $\mathrm{Zr}$ & $\mathrm{C} 13$ & $\mathrm{C} 12$ & $80.9(2)$ \\
\hline C9 & $\mathrm{Zr}$ & $\mathrm{C} 13$ & $\mathrm{C} 12$ & 109.71(19) & $\mathrm{C} 10$ & $\mathrm{Zr}$ & $\mathrm{C} 13$ & $\mathrm{C} 12$ & $-77.2(2)$ \\
\hline $\mathrm{C} 14$ & $\mathrm{Zr}$ & $\mathrm{C} 13$ & $\mathrm{C} 12$ & $-113.7(3)$ & $\mathrm{C} 11$ & $\mathrm{Zr}$ & $\mathrm{C} 13$ & $\mathrm{C} 12$ & $-36.74(18)$ \\
\hline $\mathrm{C} 1$ & $\mathrm{Zr}$ & $\mathrm{C} 13$ & $\mathrm{C} 18$ & $169.4(2)$ & $\mathrm{C} 4$ & $\mathrm{Zr}$ & $\mathrm{C} 13$ & $\mathrm{C} 18$ & $75.7(3)$ \\
\hline C7 & $\mathrm{Zr}$ & $\mathrm{C} 13$ & C18 & $-66.6(3)$ & C6 & $\mathrm{Zr}$ & $\mathrm{C} 13$ & $\mathrm{C} 18$ & $-57.4(3)$ \\
\hline C5 & $\mathrm{Zr}$ & $\mathrm{C} 13$ & $\mathrm{C} 18$ & $-11.3(3)$ & C8 & $\mathrm{Zr}$ & $\mathrm{C} 13$ & $\mathrm{C} 18$ & $-40.4(3)$ \\
\hline C12 & $\mathrm{Zr}$ & $\mathrm{C} 13$ & $\mathrm{C} 18$ & $-121.4(4)$ & C9 & $\mathrm{Zr}$ & $\mathrm{C} 13$ & $\mathrm{C} 18$ & $-11.7(3)$ \\
\hline $\mathrm{C} 10$ & $\mathrm{Zr}$ & $\mathrm{C} 13$ & $\mathrm{C} 18$ & $161.4(3)$ & C14 & $\mathrm{Zr}$ & $\mathrm{C} 13$ & $\mathrm{C} 18$ & $124.9(4)$ \\
\hline C11 & $\mathrm{Zr}$ & $\mathrm{C} 13$ & C18 & $-158.1(3)$ & C37 & $\mathrm{Si} 2$ & $\mathrm{C} 4$ & $\mathrm{C} 3$ & $-173.4(3)$ \\
\hline C35 & $\mathrm{Si} 2$ & $\mathrm{C} 4$ & C3 & $-51.6(3)$ & C36 & $\mathrm{Si} 2$ & $\mathrm{C} 4$ & $\mathrm{C} 3$ & 70.7(3) \\
\hline C37 & $\mathrm{Si} 2$ & $\mathrm{C} 4$ & $\mathrm{Zr}$ & $14.3(3)$ & $\mathrm{C} 35$ & $\mathrm{Si} 2$ & $\mathrm{C} 4$ & $\mathrm{Zr}$ & $136.1(2)$ \\
\hline C36 & Si2 & $\mathrm{C} 4$ & $\mathrm{Zr}$ & $-101.6(2)$ & $\mathrm{C} 1$ & $\mathrm{Zr}$ & $\mathrm{C} 4$ & C3 & $-4.6(2)$ \\
\hline C7 & $\mathrm{Zr}$ & $\mathrm{C} 4$ & C3 & $-101.5(2)$ & C6 & $\mathrm{Zr}$ & $\mathrm{C} 4$ & C3 & $-78.4(2)$ \\
\hline C13 & $\mathrm{Zr}$ & $\mathrm{C} 4$ & $\mathrm{C} 3$ & $131.9(2)$ & $\mathrm{C} 5$ & $\mathrm{Zr}$ & $\mathrm{C} 4$ & $\mathrm{C} 3$ & $-95.5(2)$ \\
\hline C8 & $\mathrm{Zr}$ & $\mathrm{C} 4$ & C3 & $-135.5(2)$ & $\mathrm{C} 12$ & $\mathrm{Zr}$ & $\mathrm{C} 4$ & C3 & $119.6(2)$ \\
\hline C9 & $\mathrm{Zr}$ & $\mathrm{C} 4$ & $\mathrm{C} 3$ & $-127.3(2)$ & $\mathrm{C} 10$ & $\mathrm{Zr}$ & $\mathrm{C} 4$ & C3 & $78.2(2)$ \\
\hline C14 & $\mathrm{Zr}$ & $\mathrm{C} 4$ & $\mathrm{C} 3$ & $107.4(2)$ & C11 & $\mathrm{Zr}$ & $\mathrm{C} 4$ & C3 & $79.0(2)$ \\
\hline $\mathrm{C} 1$ & $\mathrm{Zr}$ & $\mathrm{C} 4$ & $\mathrm{Si} 2$ & $168.7(2)$ & $\mathrm{C} 7$ & $\mathrm{Zr}$ & $\mathrm{C} 4$ & $\mathrm{Si} 2$ & $71.8(3)$ \\
\hline C6 & $\mathrm{Zr}$ & $\mathrm{C} 4$ & $\mathrm{Si} 2$ & $95.0(2)$ & $\mathrm{C} 13$ & $\mathrm{Zr}$ & $\mathrm{C} 4$ & $\mathrm{Si} 2$ & $-54.8(2)$ \\
\hline C5 & $\mathrm{Zr}$ & $\mathrm{C} 4$ & $\mathrm{Si} 2$ & $77.8(2)$ & C8 & $\mathrm{Zr}$ & $\mathrm{C} 4$ & $\mathrm{Si} 2$ & $37.8(2)$ \\
\hline $\mathrm{C} 12$ & $\mathrm{Zr}$ & $\mathrm{C} 4$ & $\mathrm{Si} 2$ & $-67.1(3)$ & C9 & $\mathrm{Zr}$ & $\mathrm{C} 4$ & $\mathrm{Si} 2$ & $46.0(2)$ \\
\hline $\mathrm{C} 10$ & $\mathrm{Zr}$ & $\mathrm{C} 4$ & $\mathrm{Si} 2$ & $-108.5(2)$ & C14 & $\mathrm{Zr}$ & $\mathrm{C} 4$ & $\mathrm{Si} 2$ & $-79.3(2)$ \\
\hline C11 & $\mathrm{Zr}$ & $\mathrm{C} 4$ & $\mathrm{Si} 2$ & $-107.7(2)$ & $\mathrm{Si} 2$ & $\mathrm{C} 4$ & C3 & $\mathrm{C} 2$ & $-165.3(2)$ \\
\hline $\mathrm{Zr}$ & $\mathrm{C} 4$ & C3 & $\mathrm{C} 2$ & $9.0(3)$ & $\mathrm{Si} 2$ & $\mathrm{C} 4$ & C3 & C29 & $16.2(4)$ \\
\hline $\mathrm{Zr}$ & $\mathrm{C} 4$ & $\mathrm{C} 3$ & C29 & $-169.5(2)$ & $\mathrm{C} 1$ & $\mathrm{C} 2$ & $\mathrm{C} 3$ & $\mathrm{C} 4$ & $-10.8(5)$ \\
\hline $\mathrm{C} 23$ & $\mathrm{C} 2$ & $\mathrm{C} 3$ & $\mathrm{C} 4$ & $163.3(3)$ & $\mathrm{C} 1$ & $\mathrm{C} 2$ & $\mathrm{C} 3$ & $\mathrm{C} 29$ & $167.7(3)$ \\
\hline $\mathrm{C} 23$ & $\mathrm{C} 2$ & C3 & C29 & $-18.1(4)$ & C30 & $\mathrm{C} 29$ & C3 & $\mathrm{C} 4$ & $91.0(4)$ \\
\hline
\end{tabular}




\begin{tabular}{|c|c|c|c|c|c|c|c|c|c|}
\hline C34 & $\mathrm{C} 29$ & $\mathrm{C} 3$ & $\mathrm{C} 4$ & $-88.9(4)$ & C30 & C29 & $\mathrm{C} 3$ & $\mathrm{C} 2$ & $-87.6(4)$ \\
\hline C34 & C29 & C3 & $\mathrm{C} 2$ & $92.5(4)$ & $\mathrm{C} 23$ & $\mathrm{C} 28$ & $\mathrm{C} 27$ & $\mathrm{C} 26$ & $-0.8(5)$ \\
\hline $\mathrm{C} 14$ & $\mathrm{C} 10$ & $\mathrm{C} 11$ & $\mathrm{C} 12$ & $2.7(3)$ & $\mathrm{C} 15$ & $\mathrm{C} 10$ & $\mathrm{C} 11$ & $\mathrm{C} 12$ & $175.9(3)$ \\
\hline $\mathrm{Zr}$ & $\mathrm{C} 10$ & $\mathrm{C} 11$ & $\mathrm{C} 12$ & $-64.4(2)$ & $\mathrm{C} 14$ & $\mathrm{C} 10$ & $\mathrm{C} 11$ & $\mathrm{C} 16$ & $-168.5(3)$ \\
\hline C15 & $\mathrm{C} 10$ & $\mathrm{C} 11$ & $\mathrm{C} 16$ & $4.7(5)$ & $\mathrm{Zr}$ & $\mathrm{C} 10$ & $\mathrm{C} 11$ & $\mathrm{C} 16$ & $124.4(3)$ \\
\hline C14 & $\mathrm{C} 10$ & $\mathrm{C} 11$ & $\mathrm{Zr}$ & $67.1(2)$ & $\mathrm{C} 15$ & $\mathrm{C} 10$ & $\mathrm{C} 11$ & $\mathrm{Zr}$ & $-119.7(3)$ \\
\hline $\mathrm{C} 1$ & $\mathrm{Zr}$ & $\mathrm{C} 11$ & $\mathrm{C} 12$ & $-164.1(2)$ & $\mathrm{C} 4$ & $\mathrm{Zr}$ & $\mathrm{C} 11$ & $\mathrm{C} 12$ & $114.2(2)$ \\
\hline C7 & $\mathrm{Zr}$ & $\mathrm{C} 11$ & $\mathrm{C} 12$ & $-65.4(2)$ & C6 & $\mathrm{Zr}$ & $\mathrm{C} 11$ & $\mathrm{C} 12$ & $-91.9(2)$ \\
\hline C13 & $\mathrm{Zr}$ & $\mathrm{C} 11$ & $\mathrm{C} 12$ & $37.53(19)$ & $\mathrm{C} 5$ & $\mathrm{Zr}$ & $\mathrm{C} 11$ & $\mathrm{C} 12$ & $-78.1(3)$ \\
\hline C8 & $\mathrm{Zr}$ & $\mathrm{C} 11$ & $\mathrm{C} 12$ & $-33.0(2)$ & C9 & $\mathrm{Zr}$ & $\mathrm{C} 11$ & $\mathrm{C} 12$ & $-21.4(3)$ \\
\hline C10 & $\mathrm{Zr}$ & $\mathrm{C} 11$ & $\mathrm{C} 12$ & $115.8(3)$ & C14 & $\mathrm{Zr}$ & $\mathrm{C} 11$ & $\mathrm{C} 12$ & $78.5(2)$ \\
\hline $\mathrm{C} 1$ & $\mathrm{Zr}$ & $\mathrm{C} 11$ & $\mathrm{C} 10$ & $80.1(2)$ & $\mathrm{C} 4$ & $\mathrm{Zr}$ & $\mathrm{C} 11$ & $\mathrm{C} 10$ & $-1.5(2)$ \\
\hline C7 & $\mathrm{Zr}$ & $\mathrm{C} 11$ & $\mathrm{C} 10$ & 178.81(19) & C6 & $\mathrm{Zr}$ & $\mathrm{C} 11$ & $\mathrm{C} 10$ & $152.36(18)$ \\
\hline C13 & $\mathrm{Zr}$ & $\mathrm{C} 11$ & $\mathrm{C} 10$ & $-78.2(2)$ & C5 & $\mathrm{Zr}$ & $\mathrm{C} 11$ & $\mathrm{C} 10$ & 166.1(2) \\
\hline $\mathrm{C} 8$ & $\mathrm{Zr}$ & $\mathrm{C} 11$ & $\mathrm{C} 10$ & $-148.74(19)$ & $\mathrm{C} 12$ & $\mathrm{Zr}$ & $\mathrm{C} 11$ & $\mathrm{C} 10$ & $-115.8(3)$ \\
\hline C9 & $\mathrm{Zr}$ & $\mathrm{C} 11$ & $\mathrm{C} 10$ & $-137.2(2)$ & C14 & $\mathrm{Zr}$ & $\mathrm{C} 11$ & $\mathrm{C} 10$ & $-37.28(19)$ \\
\hline $\mathrm{C} 1$ & $\mathrm{Zr}$ & $\mathrm{C} 11$ & $\mathrm{C} 16$ & $-40.9(3)$ & $\mathrm{C} 4$ & $\mathrm{Zr}$ & $\mathrm{C} 11$ & $\mathrm{C} 16$ & $-122.6(3)$ \\
\hline C7 & $\mathrm{Zr}$ & $\mathrm{C} 11$ & $\mathrm{C} 16$ & $57.8(3)$ & C6 & $\mathrm{Zr}$ & $\mathrm{C} 11$ & $\mathrm{C} 16$ & 31.3(3) \\
\hline C13 & $\mathrm{Zr}$ & $\mathrm{C} 11$ & $\mathrm{C} 16$ & $160.7(4)$ & C5 & $\mathrm{Zr}$ & $\mathrm{C} 11$ & $\mathrm{C} 16$ & $45.0(4)$ \\
\hline C8 & $\mathrm{Zr}$ & $\mathrm{C} 11$ & C16 & $90.2(3)$ & $\mathrm{C} 12$ & $\mathrm{Zr}$ & $\mathrm{C} 11$ & C16 & $123.2(4)$ \\
\hline C9 & $\mathrm{Zr}$ & $\mathrm{C} 11$ & $\mathrm{C} 16$ & 101.7(3) & $\mathrm{C} 10$ & $\mathrm{Zr}$ & $\mathrm{C} 11$ & $\mathrm{C} 16$ & $-121.0(4)$ \\
\hline C14 & $\mathrm{Zr}$ & $\mathrm{C} 11$ & $\mathrm{C} 16$ & $-158.3(4)$ & $\mathrm{C} 30$ & $\mathrm{C} 29$ & C34 & $\mathrm{C} 33$ & $-3.3(5)$ \\
\hline C3 & $\mathrm{C} 29$ & C34 & C33 & $176.6(3)$ & $\mathrm{C} 28$ & $\mathrm{C} 27$ & $\mathrm{C} 26$ & $\mathrm{C} 25$ & $1.0(5)$ \\
\hline C10 & C11 & $\mathrm{C} 12$ & $\mathrm{C} 13$ & $-2.0(3)$ & C16 & C11 & $\mathrm{C} 12$ & $\mathrm{C} 13$ & $168.9(3)$ \\
\hline $\mathrm{Zr}$ & C11 & $\mathrm{C} 12$ & $\mathrm{C} 13$ & $-66.9(2)$ & $\mathrm{C} 10$ & C11 & $\mathrm{C} 12$ & $\mathrm{C} 17$ & $-173.1(3)$ \\
\hline $\mathrm{C} 16$ & $\mathrm{C} 11$ & $\mathrm{C} 12$ & $\mathrm{C} 17$ & $-2.1(5)$ & $\mathrm{Zr}$ & $\mathrm{C} 11$ & $\mathrm{C} 12$ & $\mathrm{C} 17$ & $122.1(3)$ \\
\hline C10 & $\mathrm{C} 11$ & $\mathrm{C} 12$ & $\mathrm{Zr}$ & $64.8(2)$ & C16 & $\mathrm{C} 11$ & $\mathrm{C} 12$ & $\mathrm{Zr}$ & $-124.2(3)$ \\
\hline C14 & $\mathrm{C} 13$ & $\mathrm{C} 12$ & $\mathrm{C} 11$ & $0.6(3)$ & $\mathrm{C} 18$ & $\mathrm{C} 13$ & $\mathrm{C} 12$ & $\mathrm{C} 11$ & $-171.6(3)$ \\
\hline $\mathrm{Zr}$ & C13 & $\mathrm{C} 12$ & $\mathrm{C} 11$ & $68.3(2)$ & C14 & $\mathrm{C} 13$ & $\mathrm{C} 12$ & $\mathrm{C} 17$ & 171.7(3) \\
\hline $\mathrm{C} 18$ & $\mathrm{C} 13$ & $\mathrm{C} 12$ & $\mathrm{C} 17$ & $-0.5(5)$ & $\mathrm{Zr}$ & $\mathrm{C} 13$ & $\mathrm{C} 12$ & $\mathrm{C} 17$ & $-120.6(3)$ \\
\hline C14 & $\mathrm{C} 13$ & $\mathrm{C} 12$ & $\mathrm{Zr}$ & $-67.7(2)$ & $\mathrm{C} 18$ & $\mathrm{C} 13$ & $\mathrm{C} 12$ & $\mathrm{Zr}$ & $120.2(3)$ \\
\hline $\mathrm{C} 1$ & $\mathrm{Zr}$ & $\mathrm{C} 12$ & C11 & $18.2(2)$ & $\mathrm{C} 4$ & $\mathrm{Zr}$ & $\mathrm{C} 12$ & $\mathrm{C} 11$ & $-92.0(2)$ \\
\hline C7 & $\mathrm{Zr}$ & $\mathrm{C} 12$ & $\mathrm{C} 11$ & $116.7(2)$ & C6 & $\mathrm{Zr}$ & $\mathrm{C} 12$ & $\mathrm{C} 11$ & 107.6(2) \\
\hline C13 & $\mathrm{Zr}$ & $\mathrm{C} 12$ & $\mathrm{C} 11$ & $-114.7(3)$ & $\mathrm{C} 5$ & $\mathrm{Zr}$ & $\mathrm{C} 12$ & $\mathrm{C} 11$ & $142.0(2)$ \\
\hline C8 & $\mathrm{Zr}$ & $\mathrm{C} 12$ & $\mathrm{C} 11$ & $149.0(2)$ & C9 & $\mathrm{Zr}$ & $\mathrm{C} 12$ & $\mathrm{C} 11$ & $165.75(19)$ \\
\hline $\mathrm{C} 10$ & $\mathrm{Zr}$ & $\mathrm{C} 12$ & $\mathrm{C} 11$ & $-36.80(19)$ & C14 & $\mathrm{Zr}$ & $\mathrm{C} 12$ & $\mathrm{C} 11$ & $-77.0(2)$ \\
\hline $\mathrm{C} 1$ & $\mathrm{Zr}$ & $\mathrm{C} 12$ & $\mathrm{C} 13$ & 132.93(19) & $\mathrm{C} 4$ & $\mathrm{Zr}$ & $\mathrm{C} 12$ & $\mathrm{C} 13$ & $22.8(2)$ \\
\hline C7 & $\mathrm{Zr}$ & $\mathrm{C} 12$ & $\mathrm{C} 13$ & $-128.5(2)$ & C6 & $\mathrm{Zr}$ & $\mathrm{C} 12$ & $\mathrm{C} 13$ & $-137.61(18)$ \\
\hline C5 & $\mathrm{Zr}$ & $\mathrm{C} 12$ & $\mathrm{C} 13$ & $-103.3(2)$ & $\mathrm{C} 8$ & $\mathrm{Zr}$ & $\mathrm{C} 12$ & $\mathrm{C} 13$ & $-96.2(2)$ \\
\hline C9 & $\mathrm{Zr}$ & $\mathrm{C} 12$ & $\mathrm{C} 13$ & $-79.5(2)$ & $\mathrm{C} 10$ & $\mathrm{Zr}$ & $\mathrm{C} 12$ & $\mathrm{C} 13$ & $77.9(2)$ \\
\hline $\mathrm{C} 14$ & $\mathrm{Zr}$ & $\mathrm{C} 12$ & $\mathrm{C} 13$ & $37.76(18)$ & C11 & $\mathrm{Zr}$ & $\mathrm{C} 12$ & $\mathrm{C} 13$ & $114.7(3)$ \\
\hline $\mathrm{C} 1$ & $\mathrm{Zr}$ & $\mathrm{C} 12$ & $\mathrm{C} 17$ & $-105.5(3)$ & $\mathrm{C} 4$ & $\mathrm{Zr}$ & $\mathrm{C} 12$ & $\mathrm{C} 17$ & $144.4(3)$ \\
\hline C7 & $\mathrm{Zr}$ & $\mathrm{C} 12$ & $\mathrm{C} 17$ & $-7.0(3)$ & C6 & $\mathrm{Zr}$ & $\mathrm{C} 12$ & $\mathrm{C} 17$ & $-16.0(3)$ \\
\hline C13 & $\mathrm{Zr}$ & $\mathrm{C} 12$ & $\mathrm{C} 17$ & 121.6(4) & $\mathrm{C} 5$ & $\mathrm{Zr}$ & $\mathrm{C} 12$ & $\mathrm{C} 17$ & 18.3(4) \\
\hline C8 & $\mathrm{Zr}$ & $\mathrm{C} 12$ & $\mathrm{C} 17$ & $25.3(3)$ & C9 & $\mathrm{Zr}$ & $\mathrm{C} 12$ & $\mathrm{C} 17$ & $42.1(3)$ \\
\hline C10 & $\mathrm{Zr}$ & $\mathrm{C} 12$ & $\mathrm{C} 17$ & $-160.5(3)$ & C14 & $\mathrm{Zr}$ & $\mathrm{C} 12$ & $\mathrm{C} 17$ & $159.3(3)$ \\
\hline $\mathrm{C} 11$ & $\mathrm{Zr}$ & $\mathrm{C} 12$ & $\mathrm{C} 17$ & $-123.7(4)$ & $\mathrm{C} 27$ & $\mathrm{C} 26$ & $\mathrm{C} 25$ & $\mathrm{C} 24$ & $-1.1(5)$ \\
\hline $\mathrm{C} 26$ & $\mathrm{C} 25$ & $\mathrm{C} 24$ & $\mathrm{C} 23$ & $0.8(5)$ & $\mathrm{C} 28$ & $\mathrm{C} 23$ & $\mathrm{C} 24$ & $\mathrm{C} 25$ & $-0.5(5)$ \\
\hline $\mathrm{C} 2$ & $\mathrm{C} 23$ & $\mathrm{C} 24$ & $\mathrm{C} 25$ & $179.2(3)$ & $\mathrm{C} 34$ & $\mathrm{C} 29$ & C30 & C31 & $3.9(5)$ \\
\hline C3 & $\mathrm{C} 29$ & C30 & C31 & $-176.1(3)$ & C7 & C8 & C9 & C5 & $0.1(4)$ \\
\hline $\mathrm{Zr}$ & C8 & C9 & C5 & $65.6(2)$ & C7 & $\mathrm{C} 8$ & C9 & $\mathrm{Zr}$ & $-65.4(2)$ \\
\hline $\mathrm{C} 1$ & $\mathrm{Zr}$ & C9 & $\mathrm{C} 8$ & $113.7(2)$ & $\mathrm{C} 4$ & $\mathrm{Zr}$ & C9 & C8 & $-166.0(2)$ \\
\hline C7 & $\mathrm{Zr}$ & C9 & C8 & $37.3(2)$ & C6 & $\mathrm{Zr}$ & C9 & C8 & $78.4(2)$ \\
\hline C13 & $\mathrm{Zr}$ & C9 & $\mathrm{C} 8$ & $-65.4(2)$ & C5 & $\mathrm{Zr}$ & C9 & C8 & 115.1(3) \\
\hline $\mathrm{C} 12$ & $\mathrm{Zr}$ & C9 & $\mathrm{C} 8$ & $-32.7(2)$ & $\mathrm{C} 10$ & $\mathrm{Zr}$ & C9 & $\mathrm{C} 8$ & $-78.5(3)$ \\
\hline C14 & $\mathrm{Zr}$ & C9 & $\mathrm{C} 8$ & $-91.8(2)$ & $\mathrm{C} 11$ & $\mathrm{Zr}$ & C9 & $\mathrm{C} 8$ & $-20.8(3)$ \\
\hline
\end{tabular}




$\begin{array}{llllllllll}\text { C1 } & \text { Zr } & \text { C9 } & \text { C5 } & -1.4(3) & \text { C4 } & \text { Zr } & \text { C9 } & \text { C5 } & \text { 78.9(2) } \\ \text { C7 } & \text { Zr } & \text { C9 } & \text { C5 } & -77.8(2) & \text { C6 } & \text { Zr } & \text { C9 } & \text { C5 } & -36.7(2) \\ \text { C13 } & \text { Zr } & \text { C9 } & \text { C5 } & 179.5(2) & \text { C8 } & \text { Zr } & \text { C9 } & \text { C5 } & -115.1(3) \\ \text { C12 } & \text { Zr } & \text { C9 } & \text { C5 } & -147.8(2) & \text { C10 } & \text { Zr } & \text { C9 } & \text { C5 } & 166.3(2) \\ \text { C14 } & \text { Zr } & \text { C9 } & \text { C5 } & 153.1(2) & \text { C11 } & \text { Zr } & \text { C9 } & \text { C5 } & -135.9(2) \\ \text { C7 } & \text { C6 } & \text { C5 } & \text { C9 } & -1.0(4) & \text { Zr } & \text { C6 } & \text { C5 } & \text { C9 } & -67.1(2) \\ \text { C7 } & \text { C6 } & \text { C5 } & \text { Zr } & 66.1(2) & \text { C8 } & \text { C9 } & \text { C5 } & \text { C6 } & 0.6(4) \\ \text { Zr } & \text { C9 } & \text { C5 } & \text { C6 } & 66.2(2) & \text { C8 } & \text { C9 } & \text { C5 } & \text { Zr } & -65.6(2) \\ \text { C1 } & \text { Zr } & \text { C5 } & \text { C6 } & 63.5(2) & \text { C4 } & \text { Zr } & \text { C5 } & \text { C6 } & 147.7(2) \\ \text { C7 } & \text { Zr } & \text { C5 } & \text { C6 } & -37.7(2) & \text { C13 } & \text { Zr } & \text { C5 } & \text { C6 } & -116.1(2) \\ \text { C8 } & \text { Zr } & \text { C5 } & \text { C6 } & -78.5(2) & \text { C12 } & \text { Zr } & \text { C5 } & \text { C6 } & -69.8(3) \\ \text { C9 } & \text { Zr } & \text { C5 } & \text { C6 } & -115.3(3) & \text { C10 } & \text { Zr } & \text { C5 } & \text { C6 } & 104.1(7) \\ \text { C14 } & \text { Zr } & \text { C5 } & \text { C6 } & -163.4(2) & \text { C11 } & \text { Zr } & \text { C5 } & \text { C6 } & -22.1(4) \\ \text { C1 } & \text { Zr } & \text { C5 } & \text { C9 } & 178.8(2) & \text { C4 } & \text { Zr } & \text { C5 } & \text { C9 } & -96.9(2) \\ \text { C7 } & \text { Zr } & \text { C5 } & \text { C9 } & 77.6(2) & \text { C6 } & \text { Zr } & \text { C5 } & \text { C9 } & 115.3(3) \\ \text { C13 } & \text { Zr } & \text { C5 } & \text { C9 } & -0.7(3) & \text { C8 } & \text { Zr } & \text { C5 } & \text { C9 } & 36.8(2) \\ \text { C12 } & \text { Zr } & \text { C5 } & \text { C9 } & 45.6(3) & \text { C10 } & \text { Zr } & \text { C5 } & \text { C9 } & -140.5(6) \\ \text { C14 } & \text { Zr } & \text { C5 } & \text { C9 } & -48.0(3) & \text { C11 } & \text { Zr } & \text { C5 } & \text { C9 } & 93.3(3) \\ \text { C29 } & \text { C30 } & \text { C31 } & \text { C32 } & -1.5(6) & \text { C29 } & \text { C34 } & \text { C33 } & \text { C32 } & 0.4(6) \\ \text { C34 } & \text { C33 } & \text { C32 } & \text { C31 } & 2.0(6) & \text { C30 } & \text { C31 } & \text { C32 } & \text { C33 } & -1.4(6)\end{array}$

\section{X-Ray Structural Data for 2:}

\section{$\underline{\text { Data Collection }}$}

A fragment of a yellow plate-like crystal of $\mathrm{C}_{77} \mathrm{H}_{84} \mathrm{Si}_{4} \mathrm{Zr}_{2}$ having approximate dimensions of $0.1 \times 0.1 \times 0.05 \mathrm{~mm}$ was mounted on a glass fiber using Paratone $\mathrm{N}$ hydrocarbon oil. All measurements were made on a Bruker P4 CCD area detector with graphite monochromated MoK radiation.

Cell constants and an orientation matrix, obtained from a least-squares refinement using the measured positions of 1826 centered reflections with $\mathrm{I}>10 \sigma(\mathrm{I})$ in the range $2.78<\theta<21.73^{\circ}$ corresponded to a primitive triclinic cell with dimensions:

$$
\begin{array}{ll}
\mathrm{a}=7.507(2) \AA & \alpha=78.214(4)^{\mathrm{o}} \\
\mathrm{b}=11.123(3) \AA & \beta=83.497(4)^{\mathrm{o}} \\
\mathrm{c}=21.788(5) \AA & \gamma=77.487(4)^{\circ} \\
\mathrm{V}=8876(1) \AA^{3} &
\end{array}
$$

For $\mathrm{Z}=1$ and F.W. $=1304.04$, the calculated density is $1.249 \mathrm{~g} / \mathrm{cm}^{3}$.

Analysis of the systematic absences allowed the space group to be uniquely determined to be:

$\mathrm{P}-1$

The data were collected at a temperature of 193(2) K. Frames corresponding to an arbitrary hemisphere of data were collected using $\omega$ scans of $0.3^{\circ}$ counted for a total of 10 seconds per frame. 


\section{Data Reduction}

Data were integrated by the program $\operatorname{SAINT}^{1}$ to a maximum $\theta$ value of $23.25^{\circ}$. The data were corrected for Lorentz and polarization effects. Data were analyzed for agreement and possible absorption using XPREP ${ }^{2}$. An empirical absorption correction based on comparison of redundant and equivalent reflections was applied using $\mathrm{SADABS}^{3} .\left(\mathrm{T}_{\max }=0.980, \mathrm{~T}_{\min }=0.900\right)$. Of the 8810 reflections that were collected, 4978 were unique $\left(\mathrm{R}_{\text {int }}=0.0464\right)$; equivalent reflections were merged. No decay correction was applied.

\section{$\underline{\text { Structure Solution and Refinement }}$}

The structure was solved by direct methods ${ }^{4}$ and expanded using Fourier techniques ${ }^{5}$. Some non-hydrogen atoms were refined anisotropically, while the rest were refined isotropically. Hydrogen atoms were included but not refined. The final cycle of full-matrix least-squares refinement ${ }^{6}$ was based on 4978 reflections (all data) and 363 variable parameters and converged (largest parameter shift was 0.000 times its esd) with conventional unweighted and weighted agreement factors of:

$\mathrm{R} 1=\Sigma\left\|\mathrm{F}_{\mathrm{o}}|-| \mathrm{F}_{\mathrm{c}}\right\| / \Sigma\left|\mathrm{F}_{\mathrm{o}}\right|=0.0402$ for 3152 data with $\mathrm{I}>2 \sigma(\mathrm{I})$

$\mathrm{wR} 2=\left[\left(\Sigma \mathrm{w}\left(\left|\mathrm{F}_{\mathrm{o}}\right|^{2}-\left|\mathrm{F}_{\mathrm{c}}\right|^{2}\right)^{2} / \Sigma \mathrm{w} \mid \mathrm{Fo}^{2}\right)\right]^{1 / 2}=0.0825$

The standard deviation of an observation of unit weight ${ }^{7}$ was 0.821 . The weighting scheme was based on counting statistics and included a factor to downweight the intense reflections. The maximum and minimum peaks on the final difference Fourier map corresponded to 0.632 and -0.346 e- $/ \AA^{3}$, respectively.

Neutral atom scattering factors were taken from Cromer and Waber ${ }^{8}$. Anomalous dispersion effects were included in Fcalc ${ }^{6}$; the values for Df' and Df" were those of Creagh and McAuley ${ }^{9}$. The values for the mass attenuation coefficients are those of Creagh and Hubbel ${ }^{10}$. All calculations were performed using the SHELXTL ${ }^{11}$ crystallographic software package of Bruker Analytical X-ray Systems Inc.

\section{Experimental Details:}

\section{A. Crystal Data}

Empirical Formula

Formula Weight

Crystal Color, Habit

Crystal Dimensions

Crystal System

Lattice Type
$\mathrm{C}_{77} \mathrm{H}_{84} \mathrm{Si}_{4} \mathrm{Zr}_{2}$

1304.04

yellow, plate

0.1 x 0.1 x $0.05 \mathrm{~mm}$

triclinic

primitive 
Lattice Parameters

Space Group

$\mathrm{Z}$ value

$\mathrm{D}_{\text {calc }}$

$\mathrm{F}_{000}$

$\mu(\mathrm{MoK})$

B. Intensity Measurements

Diffractometer

Radiation

Detector Position

Exposure Time

Scan Type

$\theta$ max

No. of Reflections Measured

Corrections

C. Structure Solution and Refinement

Structure Solution

Refinement

Function Minimized

Least Squares Weighting scheme

Anomalous Dispersion

No. Observations $(\mathrm{I}>2.00 \sigma(\mathrm{I}))$

No. Variables

Residuals: R; $\mathrm{wR}_{2}$; Rall

Goodness of Fit Indicator

Max Shift/Error in Final Cycle

Maximum peak in Final Diff. Map

Minimum peak in Final Diff. Map

$$
\begin{aligned}
& \mathrm{a}=7.507(2) \AA \\
& \mathrm{b}=11.123(3) \AA \\
& \mathrm{c}=21.788(5) \AA \\
& \alpha=78.214(4)^{\mathrm{o}} \\
& \beta=83.497(4)^{\mathrm{O}} \\
& \gamma=77.487(4)^{\mathrm{o}} \\
& \mathrm{V}=1734.1(7) \AA^{3} \\
& \mathrm{P}-1 \\
& 1 \\
& 1.249 \mathrm{~g} / \mathrm{cm}^{3} \\
& 640 \\
& 0.41 \mathrm{~cm}^{-1}
\end{aligned}
$$

Bruker P4

$\operatorname{MoK}(\lambda=0.71073 \AA)$

graphite monochromated

$60.00 \mathrm{~mm}$

10 seconds per frame.

$\omega$ (0.3 degrees per frame)

$23.25^{\circ}$

Total: 8810

Unique: $4978\left(\mathrm{R}_{\text {int }}=0.0464\right)$

Lorentz-polarization

Absorption $\left(\mathrm{T}_{\max }=0.980\right.$,

$\mathrm{T}_{\min }=0.900$ )

direct (SHELXS-97 (Sheldrick, 1990))

Full-matrix least-squares

$\sum w\left(|\mathrm{Fo}|^{2}-\mid \mathrm{Fc}^{2}\right)^{2}$

$\mathrm{w}=1 /\left[\sigma^{2}\left(\mathrm{~F}_{\mathrm{o}}^{2}\right)+(\mathrm{qP})^{2}+0.000 \mathrm{P}\right]$

where $\mathrm{P}=\left[\mathrm{F}_{\mathrm{o}}{ }^{2}+2 \mathrm{~F}_{\mathrm{c}}{ }^{2}\right] / 3$

All non-hydrogen atoms

3152

363

$0.0402 ; 0.0825 ; 0.0617$

0.821

0.000

$0.632 \mathrm{e}-/ \AA^{3}$

$-0.346 \mathrm{e}-/ \AA^{3}$ 
Table S7. Atomic coordinates and $\mathrm{U}_{\mathrm{iso}} / \mathrm{U}_{\text {eq }}$ and occupancy for $2 . \mathrm{U}_{\mathrm{eq}}$ is defined as one third of the trace of the orthogonalized $\mathrm{U}_{\mathrm{ij}}$ tensor.

\begin{tabular}{|c|c|c|c|c|c|}
\hline atom & $\mathbf{X}$ & $\mathbf{y}$ & $\mathbf{Z}$ & $\mathbf{U}_{\mathbf{e q}}$ & Occupancy \\
\hline Zr & $-0.0268(1)$ & $-0.1881(1)$ & $-0.1794(1)$ & $0.023(1)$ & 1 \\
\hline Sil & $-0.1808(2)$ & $0.1253(1)$ & $-0.1258(1)$ & $0.030(1)$ & 1 \\
\hline Si2 & $-0.0931(2)$ & $-0.2799(1)$ & $-0.3314(1)$ & $0.031(1)$ & 1 \\
\hline $\mathrm{C} 1$ & $-0.1556(5)$ & $0.0145(4)$ & $-0.1820(2)$ & $0.024(1)$ & 1 \\
\hline $\mathrm{C} 2$ & $-0.2309(5)$ & $0.0407(4)$ & $-0.2377(2)$ & $0.024(1)$ & 1 \\
\hline $\mathrm{C} 3$ & $-0.2050(5)$ & $-0.0484(4)$ & $-0.2849(2)$ & $0.024(1)$ & 1 \\
\hline $\mathrm{C} 4$ & $-0.1247(5)$ & $-0.1726(4)$ & $-0.2737(2)$ & $0.024(1)$ & 1 \\
\hline $\mathrm{C} 5$ & 0.2669 (5) & $-0.3055(4)$ & $-0.1389(2)$ & $0.026(1)$ & 1 \\
\hline $\mathrm{C} 6$ & $0.2785(5)$ & $-0.3186(4)$ & $-0.2022(2)$ & $0.030(1)$ & 1 \\
\hline $\mathrm{C} 7$ & $0.2868(6)$ & $-0.2016(5)$ & $-0.2409(2)$ & $0.032(1)$ & 1 \\
\hline $\mathrm{C} 8$ & $0.2756(5)$ & $-0.1162(5)$ & $-0.2017(2)$ & $0.035(1)$ & 1 \\
\hline C9 & $0.2638(5)$ & $-0.1782(5)$ & $-0.1387(2)$ & $0.031(1)$ & 1 \\
\hline C10 & $0.2022(6)$ & $-0.3976(4)$ & $-0.0823(2)$ & $0.031(1)$ & 1 \\
\hline C11 & $0.2585(6)$ & $-0.3762(5)$ & $-0.0202(2)$ & $0.045(2)$ & 1 \\
\hline C12 & $0.2764(6)$ & $-0.5335(4)$ & $-0.0899(2)$ & $0.047(1)$ & 1 \\
\hline C13 & $-0.0060(6)$ & $-0.3591(4)$ & $-0.0868(2)$ & $0.025(1)$ & 1 \\
\hline C14 & $-0.1018(6)$ & $-0.3946(4)$ & $-0.1304(2)$ & $0.028(1)$ & 1 \\
\hline C15 & $-0.2720(6)$ & $-0.3146(4)$ & $-0.1357(2)$ & $0.031(1)$ & 1 \\
\hline C16 & $-0.2852(6)$ & $-0.2279(4)$ & $-0.0966(2)$ & $0.030(1)$ & 1 \\
\hline C17 & $-0.1195(6)$ & $-0.2554(4)$ & $-0.0666(2)$ & $0.027(1)$ & 1 \\
\hline C18 & $0.0361(6)$ & $0.0973(5)$ & $-0.0860(2)$ & $0.046(1)$ & 1 \\
\hline C19 & $-0.2238(6)$ & $0.2964(4)$ & $-0.1586(2)$ & $0.041(1)$ & 1 \\
\hline $\mathrm{C} 20$ & $-0.3675(6)$ & $0.0975(4)$ & $-0.0632(2)$ & $0.043(1)$ & 1 \\
\hline C21 & $-0.3585(6)$ & $0.1651(4)$ & $-0.2580(2)$ & $0.025(1)$ & 1 \\
\hline C22 & $-0.3059(6)$ & $0.2607(4)$ & $-0.3037(2)$ & $0.034(1)$ & 1 \\
\hline C23 & $-0.4269(7)$ & $0.3705(5)$ & $-0.3200(2)$ & $0.044(1)$ & 1 \\
\hline C24 & $-0.6030(8)$ & $0.3865(5)$ & $-0.2928(2)$ & $0.051(2)$ & 1 \\
\hline C25 & $-0.6645(7)$ & $0.2952(5)$ & $-0.2483(2)$ & $0.044(1)$ & 1 \\
\hline C26 & $-0.5335(6)$ & $0.1846(4)$ & $-0.2301(2)$ & $0.033(1)$ & 1 \\
\hline $\mathrm{C} 27$ & $-0.2709(6)$ & $0.0135(4)$ & $-0.3487(2)$ & $0.027(1)$ & 1 \\
\hline C2 8 & $-0.1436(6)$ & $0.0464(4)$ & $-0.3979(2)$ & $0.034(1)$ & 1 \\
\hline C29 & $-0.1970(7)$ & $0.0989(5)$ & $-0.4574(2)$ & $0.046(1)$ & 1 \\
\hline C3O & $-0.3773(8)$ & $0.1191(5)$ & $-0.4696(2)$ & $0.053(2)$ & 1 \\
\hline C31 & $-0.5037(7)$ & $0.0875(5)$ & $-0.4219(2)$ & $0.045(1)$ & 1 \\
\hline C32 & $-0.4520(6)$ & $0.0361(4)$ & $-0.3619(2)$ & $0.035(1)$ & 1 \\
\hline C33 & $-0.0921(6)$ & $-0.4423(4)$ & $-0.2865(2)$ & $0.039(1)$ & 1 \\
\hline C34 & $-0.2703(6)$ & $-0.2510(4)$ & $-0.3896(2)$ & $0.050(2)$ & 1 \\
\hline C35 & $0.1354(6)$ & $-0.2798(4)$ & $-0.3756(2)$ & $0.043(1)$ & 1 \\
\hline C100 & $-0.1040(16)$ & $-0.3736(11)$ & $-0.5572(5)$ & $0.058(3)$ & 0.50 \\
\hline C101 & $0.0527(10)$ & $-0.4120(6)$ & $-0.5353(3)$ & $0.079(2)$ & 1 \\
\hline C102 & $0.2008(17)$ & $-0.4567(13)$ & $-0.5122(6)$ & $0.073(4)$ & 0.50 \\
\hline C103 & $0.1122(17)$ & $-0.5175(13)$ & $-0.4888(6)$ & $0.072(4)$ & 0.50 \\
\hline C104 & $0.2685(11)$ & $-0.5656(8)$ & $-0.4645(4)$ & $0.120(3)$ & 1 \\
\hline
\end{tabular}

Table S8. Bond lengths $(\AA)$ for 2 .

$\begin{array}{lllllllll}\text { atom } & \text { atom } & \text { distance } & \text { atom } & \text { atom } & \text { distance } & \text { atom } & \text { atom } & \text { distance } \\ \mathrm{Zr} & \mathrm{C} 4 & 2.221(4) & \mathrm{Zr} & \mathrm{C} 1 & 2.243(4) & \mathrm{Zr} & \mathrm{C} 5 & 2.463(4) \\ \mathrm{Zr} & \mathrm{C} 13 & 2.468(4) & \mathrm{Zr} & \mathrm{C} 9 & 2.478(4) & \mathrm{Zr} & \mathrm{C} 14 & 2.482(4) \\ \mathrm{Zr} & \mathrm{C} 17 & 2.485(4) & \mathrm{Zr} & \mathrm{C} 6 & 2.491(4) & \mathrm{Zr} & \mathrm{C} 8 & 2.531(4) \\ \mathrm{Zr} & \mathrm{C} 15 & 2.540(4) & \mathrm{Zr} & \mathrm{C} 16 & 2.543(4) & \mathrm{Zr} & \mathrm{C} 7 & 2.564(4)\end{array}$




\begin{tabular}{|c|c|c|c|c|c|c|c|c|}
\hline Si1 & C19 & $1.862(4)$ & Si1 & C18 & $1.868(4)$ & Si1 & $\mathrm{C} 20$ & $1.872(4)$ \\
\hline Si1 & C1 & $1.875(4)$ & Si2 & C4 & $1.867(4)$ & Si2 & C35 & $1.870(4)$ \\
\hline Si2 & C33 & $1.868(4)$ & Si2 & C34 & $1.873(4)$ & C1 & C2 & $1.348(5)$ \\
\hline C2 & $\mathrm{C} 21$ & $1.516(6)$ & $\mathrm{C} 2$ & C3 & $1.538(6)$ & C3 & C4 & $1.366(6)$ \\
\hline C3 & $\mathrm{C} 27$ & $1.507(5)$ & C5 & C6 & $1.408(5)$ & C5 & C9 & $1.413(6)$ \\
\hline C5 & C10 & $1.540(6)$ & C6 & C7 & $1.408(6)$ & C6 & $\mathrm{H} 6 \mathrm{~A}$ & 0.9800 \\
\hline C7 & $\mathrm{C} 8$ & $1.386(6)$ & C7 & H7A & 0.9800 & $\mathrm{C} 8$ & C9 & $1.407(5)$ \\
\hline C8 & $\mathrm{H} 8 \mathrm{~A}$ & 0.9800 & C9 & H9A & 0.9800 & $\mathrm{C} 10$ & C12 & $1.529(6)$ \\
\hline $\mathrm{C} 10$ & C13 & $1.537(6)$ & C10 & C11 & $1.540(5)$ & $\mathrm{C} 11$ & $\mathrm{H} 11 \mathrm{~A}$ & 0.9600 \\
\hline C11 & H11B & 0.9600 & $\mathrm{C} 11$ & $\mathrm{H} 11 \mathrm{C}$ & 0.9600 & $\mathrm{C} 12$ & $\mathrm{H} 12 \mathrm{~A}$ & 0.9600 \\
\hline C12 & $\mathrm{H} 12 \mathrm{~B}$ & 0.9600 & $\mathrm{C} 12$ & $\mathrm{H} 12 \mathrm{C}$ & 0.9600 & C13 & C17 & $1.395(6)$ \\
\hline $\mathrm{C} 13$ & C14 & $1.418(5)$ & C14 & C15 & $1.392(6)$ & C14 & $\mathrm{H} 14 \mathrm{~A}$ & 0.9800 \\
\hline C15 & C16 & $1.394(6)$ & C15 & H15A & 0.9800 & C16 & C17 & $1.416(5)$ \\
\hline C16 & $\mathrm{H16A}$ & 0.9800 & C17 & H17A & 0.9800 & C18 & H18A & 0.9600 \\
\hline C18 & H18B & 0.9600 & C18 & $\mathrm{H} 18 \mathrm{C}$ & 0.9600 & C19 & H19A & 0.9600 \\
\hline C19 & H19B & 0.9600 & C19 & $\mathrm{H} 19 \mathrm{C}$ & 0.9600 & C20 & $\mathrm{H} 20 \mathrm{~A}$ & 0.9600 \\
\hline C20 & $\mathrm{H} 20 \mathrm{~B}$ & 0.9600 & $\mathrm{C} 20$ & $\mathrm{H} 20 \mathrm{C}$ & 0.9600 & C21 & C26 & $1.375(5)$ \\
\hline C21 & C22 & $1.393(6)$ & $\mathrm{C} 22$ & C23 & $1.363(6)$ & C22 & H2 2A & 0.9300 \\
\hline C23 & $\mathrm{C} 24$ & $1.376(6)$ & $\mathrm{C} 23$ & H23A & 0.9300 & C24 & C25 & $1.373(6)$ \\
\hline C24 & $\mathrm{H} 24 \mathrm{~A}$ & 0.9300 & C25 & C26 & $1.415(6)$ & C25 & H25A & 0.9300 \\
\hline C26 & $\mathrm{H} 26 \mathrm{~A}$ & 0.9300 & $\mathrm{C} 27$ & C32 & $1.380(5)$ & C27 & C2 8 & $1.399(5)$ \\
\hline C2 8 & C29 & $1.373(6)$ & $\mathrm{C} 28$ & H2 8A & 0.9300 & C29 & C30 & $1.372(6)$ \\
\hline C29 & H29A & 0.9300 & $\mathrm{C} 30$ & C31 & $1.370(6)$ & C30 & $\mathrm{H} 30 \mathrm{~A}$ & 0.9300 \\
\hline C31 & C32 & $1.379(6)$ & C31 & H31A & 0.9300 & C32 & H32A & 0.9300 \\
\hline C33 & H33A & 0.9600 & C33 & H33 B & 0.9600 & C33 & H3 3C & 0.9600 \\
\hline C34 & H3 4A & 0.9600 & C34 & H3 4B & 0.9600 & C34 & $\mathrm{H} 34 \mathrm{C}$ & 0.9600 \\
\hline C35 & H35A & 0.9600 & C35 & H3 5B & 0.9600 & C35 & H3 5C & 0.9600 \\
\hline $\mathrm{C} 100$ & C101 & $1.272(11)$ & $\mathrm{C} 100$ & C103\#1 & $1.414(16)$ & $\mathrm{C} 100$ & C104\#1 & $1.518(12)$ \\
\hline C101 & $\mathrm{C} 102$ & $1.233(13)$ & C101 & C103 & $1.412(14)$ & C101 & C103\#1 & $1.591(13)$ \\
\hline $\mathrm{C} 102$ & $\mathrm{C} 103$ & $1.065(15)$ & C102 & C104 & $1.465(14)$ & $\mathrm{C} 103$ & C104 & $1.302(13)$ \\
\hline $\mathrm{C} 103$ & C100\#1 & $-1.414(16)$ & $\mathrm{C} 103$ & C101\#1 & $1.591(13)$ & $\mathrm{C} 103$ & C103\#1 & $1.75(2)$ \\
\hline C104 & C100\#1 1. & $.518(12)$ & & & & & & \\
\hline
\end{tabular}

Table S9. Bond angles $\left(^{\circ}\right)$ for 2.

$\begin{array}{llllllll}\text { atom } & \text { atom } & \text { atom } & \text { angle } & \text { atom } & \text { atom } & \text { atom } & \text { angle } \\ \text { C4 } & \text { Zr } & \text { C1 } & 88.83(15) & \text { C4 } & \text { Zr } & \text { C5 } & 128.08(15) \\ \text { C1 } & \text { Zr } & \text { C5 } & 128.85(15) & \text { C4 } & \text { Zr } & \text { C13 } & 131.24(15) \\ \text { C1 } & \text { Zr } & \text { C13 } & 127.23(15) & \text { C5 } & \text { Zr } & \text { C13 } & 57.17(14) \\ \text { C4 } & \text { Zr } & \text { C9 } & 135.71(15) & \text { C1 } & \text { Zr } & \text { C9 } & 95.94(15) \\ \text { C5 } & \text { Zr } & \text { C9 } & 33.22(13) & \text { C13 } & \text { Zr } & \text { C9 } & 78.29(14) \\ \text { C4 } & \text { Zr } & \text { C14 } & 98.16(14) & \text { C1 } & \text { Zr } & \text { C14 } & 137.18(15) \\ \text { C5 } & \text { Zr } & \text { C14 } & 77.96(14) & \text { C13 } & \text { Zr } & \text { C14 } & 33.30(12) \\ \text { C9 } & \text { Zr } & \text { C14 } & 107.21(15) & \text { C4 } & \text { Zr } & \text { C17 } & 139.79(14) \\ \text { C1 } & \text { Zr } & \text { C17 } & 94.77(15) & \text { C5 } & \text { Zr } & \text { C17 } & 78.65(14) \\ \text { C13 } & \text { Zr } & \text { C17 } & 32.72(13) & \text { C9 } & \text { Zr } & \text { C17 } & 83.80(14) \\ \text { C14 } & \text { Zr } & \text { C17 } & 54.09(14) & \text { C4 } & \text { Zr } & \text { C6 } & 95.12(15) \\ \text { C1 } & \text { Zr } & \text { C6 } & 138.90(15) & \text { C5 } & \text { Zr } & \text { C6 } & 33.03(13) \\ \text { C13 } & \text { Zr } & \text { C6 } & 78.43(14) & \text { C9 } & \text { Zr } & \text { C6 } & 54.33(15) \\ \text { C14 } & \text { Zr } & \text { C6 } & 82.77(15) & \text { C17 } & \text { Zr } & \text { C6 } & 107.49(14) \\ \text { C4 } & \text { Zr } & \text { C8 } & 104.59(15) & \text { C1 } & \text { Zr } & \text { C8 } & 86.16(15) \\ \text { C5 } & \text { Zr } & \text { C8 } & 54.17(14) & \text { C13 } & \text { Zr } & \text { C8 } & 108.94(14) \\ \text { C9 } & \text { Zr } & \text { C8 } & 32.61(13) & \text { C14 } & \text { Zr } & \text { C8 } & 131.41(15) \\ \text { C17 } & \text { Zr } & \text { C8 } & 115.60(14) & \text { C6 } & \text { Zr } & \text { C8 } & 53.23(15) \\ \text { C4 } & \text { Zr } & \text { C15 } & 87.08(14) & \text { C1 } & \text { Zr } & \text { C15 } & 107.22(15)\end{array}$




\begin{tabular}{|c|c|c|c|c|c|c|c|}
\hline C5 & Zr & C15 & $108.50(14)$ & C13 & Zr & C15 & $54.21(13)$ \\
\hline C9 & Zr & C15 & $132.10(14)$ & C14 & $\mathrm{Zr}$ & C15 & $32.15(13)$ \\
\hline C17 & Zr & C15 & $53.60(14)$ & C6 & Zr & C15 & $113.82(15)$ \\
\hline C8 & Zr & C15 & $162.63(14)$ & C4 & Zr & C16 & $108.65(14)$ \\
\hline C1 & Zr & C16 & $84.25(15)$ & C5 & Zr & C16 & $109.33(14)$ \\
\hline C13 & Zr & C16 & $54.22(14)$ & C9 & Zr & C16 & $115.63(14)$ \\
\hline C14 & Zr & C16 & $53.41(15)$ & C17 & Zr & C16 & $32.70(12)$ \\
\hline C6 & Zr & C16 & $131.81(15)$ & C8 & Zr & C16 & $145.11(15)$ \\
\hline C15 & Zr & C16 & $31.83(13)$ & C4 & Zr & C7 & $83.05(14)$ \\
\hline C1 & Zr & C7 & $108.69(15)$ & C5 & Zr & C7 & $54.18(14)$ \\
\hline C13 & Zr & C7 & $108.93(14)$ & C9 & Zr & $\mathrm{C} 7$ & $53.69(14)$ \\
\hline C14 & Zr & C7 & $114.08(15)$ & C17 & Zr & C7 & $132.32(14)$ \\
\hline C6 & Zr & C7 & 32.31 (13) & $\mathrm{C} 8$ & Zr & C7 & $31.57(13)$ \\
\hline C15 & Zr & C7 & $142.45(15)$ & $\mathrm{C} 16$ & Zr & C7 & $163.11(14)$ \\
\hline C19 & Si1 & C18 & $103.7(2)$ & C19 & Si1 & $\mathrm{C} 20$ & $107.2(2)$ \\
\hline C18 & Sil & $\mathrm{C} 20$ & $107.4(2)$ & C19 & Sil & C1 & $117.88(19)$ \\
\hline C18 & Si1 & C1 & $109.42(19)$ & $\mathrm{C} 20$ & Sil & $\mathrm{C} 1$ & $110.62(19)$ \\
\hline C4 & Si2 & C35 & $109.01(19)$ & $\mathrm{C} 4$ & Si2 & C33 & $108.06(19)$ \\
\hline C35 & Si2 & C33 & $107.6(2)$ & C4 & Si2 & C34 & $117.9(2)$ \\
\hline C35 & Si2 & C34 & $108.4(2)$ & C33 & Si2 & C34 & $105.4(2)$ \\
\hline C2 & C1 & Si1 & $125.5(3)$ & $\mathrm{C} 2$ & $\mathrm{C} 1$ & Zr & $99.7(3)$ \\
\hline Si1 & $\mathrm{C} 1$ & Zr & $134.6(2)$ & $\mathrm{C} 1$ & C2 & $\mathrm{C} 21$ & $120.6(4)$ \\
\hline C1 & C2 & C3 & $125.6(4)$ & C21 & $\mathrm{C} 2$ & C3 & $113.8(3)$ \\
\hline C1 & $\mathrm{C} 2$ & Zr & $52.0(2)$ & C21 & $\mathrm{C} 2$ & Zr & $169.9(3)$ \\
\hline C3 & $\mathrm{C} 2$ & Zr & $73.8(2)$ & $\mathrm{C} 4$ & C3 & $\mathrm{C} 27$ & $120.1(4)$ \\
\hline C4 & C3 & $\mathrm{C} 2$ & $125.6(4)$ & $\mathrm{C} 27$ & C3 & C2 & $114.2(4)$ \\
\hline $\mathrm{C} 4$ & $\mathrm{C} 3$ & Zr & $51.5(2)$ & $\mathrm{C} 27$ & C3 & Zr & $169.0(3)$ \\
\hline $\mathrm{C} 2$ & C3 & Zr & $74.3(2)$ & C3 & C4 & Si2 & $125.3(3)$ \\
\hline C3 & C4 & Zr & $99.7(3)$ & Si2 & C4 & Zr & $134.6(2)$ \\
\hline C6 & C5 & C9 & $107.0(4)$ & C6 & C5 & C10 & $125.5(4)$ \\
\hline C9 & C5 & $\mathrm{C} 10$ & $124.3(4)$ & C6 & C5 & $\mathrm{Zr}$ & $74.5(2)$ \\
\hline C9 & C5 & Zr & $73.9(2)$ & $\mathrm{C} 10$ & C5 & Zr & $101.4(3)$ \\
\hline C5 & C6 & C7 & $108.9(4)$ & C5 & C6 & Zr & $72.4(2)$ \\
\hline C7 & C6 & $\mathrm{Zr}$ & $76.7(3)$ & C5 & C6 & $\mathrm{H} 6 \mathrm{~A}$ & 125.2 \\
\hline C7 & C6 & $\mathrm{H} 6 \mathrm{~A}$ & 125.2 & Zr & C6 & $\mathrm{H} 6 \mathrm{~A}$ & 125.2 \\
\hline C8 & C7 & C6 & $107.3(4)$ & C8 & C7 & Zr & $72.9(3)$ \\
\hline C6 & C7 & Zr & $71.0(2)$ & $\mathrm{C} 8$ & C7 & H7A & 126.2 \\
\hline C6 & C7 & H7A & 126.2 & Zr & C7 & H7A & 126.2 \\
\hline C7 & C8 & C9 & $109.3(4)$ & C7 & $\mathrm{C} 8$ & Zr & $75.5(3)$ \\
\hline C9 & C8 & Zr & $71.6(2)$ & C7 & C8 & H8A & 125.2 \\
\hline C9 & $\mathrm{C} 8$ & H8A & 125.2 & Zr & $\mathrm{C} 8$ & $\mathrm{H} 8 \mathrm{~A}$ & 125.2 \\
\hline C8 & C9 & C5 & $107.6(4)$ & C8 & C9 & Zr & $75.8(2)$ \\
\hline C5 & C9 & Zr & $72.8(2)$ & $\mathrm{C} 8$ & C9 & H9A & 125.8 \\
\hline C5 & C9 & H9A & 125.8 & Zr & C9 & H9A & 125.8 \\
\hline C12 & C10 & C13 & $112.5(4)$ & C12 & C10 & C5 & $111.1(4)$ \\
\hline C13 & C10 & C5 & $100.2(3)$ & $\mathrm{C} 12$ & $\mathrm{C} 10$ & $\mathrm{C} 11$ & $110.0(4)$ \\
\hline C13 & C10 & C11 & $111.4(4)$ & C5 & C10 & C11 & $111.3(4)$ \\
\hline C10 & C11 & $\mathrm{H} 11 \mathrm{~A}$ & 109.5 & C10 & C11 & $\mathrm{H} 11 \mathrm{~B}$ & 109.5 \\
\hline H11A & C11 & H11B & 109.5 & C10 & $\mathrm{C} 11$ & $\mathrm{H} 11 \mathrm{C}$ & 109.5 \\
\hline H11A & C11 & $\mathrm{H} 11 \mathrm{C}$ & 109.5 & $\mathrm{H} 11 \mathrm{~B}$ & C11 & $\mathrm{H} 11 \mathrm{C}$ & 109.5 \\
\hline C10 & C12 & $\mathrm{H} 12 \mathrm{~A}$ & 109.5 & C10 & $\mathrm{C} 12$ & $\mathrm{H} 12 \mathrm{~B}$ & 109.5 \\
\hline $\mathrm{H} 12 \mathrm{~A}$ & $\mathrm{C} 12$ & $\mathrm{H} 12 \mathrm{~B}$ & 109.5 & C10 & $\mathrm{C} 12$ & $\mathrm{H} 12 \mathrm{C}$ & 109.5 \\
\hline $\mathrm{H} 12 \mathrm{~A}$ & C12 & $\mathrm{H} 12 \mathrm{C}$ & 109.5 & $\mathrm{H} 12 \mathrm{~B}$ & $\mathrm{C} 12$ & $\mathrm{H} 12 \mathrm{C}$ & 109.5 \\
\hline C17 & C13 & C14 & $106.8(4)$ & C17 & $\mathrm{C} 13$ & C10 & $126.0(4)$ \\
\hline C14 & C13 & C10 & $123.9(4)$ & C17 & $\mathrm{C} 13$ & $\mathrm{Zr}$ & $74.3(2)$ \\
\hline C14 & C13 & $\mathrm{Zr}$ & $73.9(2)$ & C10 & $\mathrm{C} 13$ & Zr & $101.3(3)$ \\
\hline C15 & C14 & C13 & $108.7(4)$ & C15 & C14 & Zr & $76.2(3)$ \\
\hline
\end{tabular}




\begin{tabular}{|c|c|c|c|c|c|c|c|}
\hline C13 & C14 & Zr & $72.8(2)$ & C15 & C14 & $\mathrm{H} 14 \mathrm{~A}$ & 125.3 \\
\hline C13 & C14 & $\mathrm{H} 14 \mathrm{~A}$ & 125.3 & Zr & C14 & $\mathrm{H} 14 \mathrm{~A}$ & 125.3 \\
\hline C14 & C15 & C16 & $108.3(4)$ & $\mathrm{C} 14$ & C15 & Zr & $71.6(2)$ \\
\hline C16 & C15 & Zr & $74.2(2)$ & C14 & C15 & H15A & 125.6 \\
\hline C16 & C15 & H15A & 125.6 & $\mathrm{Zr}$ & C15 & H15A & 125.6 \\
\hline C15 & C16 & C17 & $107.5(4)$ & C15 & C16 & Zr & $74.0(2)$ \\
\hline C17 & C16 & Zr & $71.4(2)$ & C15 & C16 & H16A & 126.0 \\
\hline C17 & C16 & $\mathrm{H} 16 \mathrm{~A}$ & 126.0 & Zr & $\mathrm{C} 16$ & $\mathrm{H} 16 \mathrm{~A}$ & 126.0 \\
\hline C13 & C17 & C16 & $108.7(4)$ & $\mathrm{C} 13$ & C17 & Zr & $73.0(2)$ \\
\hline C16 & C17 & Zr & $75.9(2)$ & $\mathrm{C} 13$ & C17 & H17A & 125.3 \\
\hline C16 & C17 & H17A & 125.3 & Zr & C17 & H17A & 125.3 \\
\hline Si1 & C18 & $\mathrm{H} 18 \mathrm{~A}$ & 109.5 & Si1 & C18 & H18B & 109.5 \\
\hline H18A & C18 & H18B & 109.5 & Si1 & $\mathrm{C} 18$ & H1 8C & 109.5 \\
\hline H18A & C18 & $\mathrm{H} 18 \mathrm{C}$ & 109.5 & $\mathrm{H} 18 \mathrm{~B}$ & C18 & $\mathrm{H} 18 \mathrm{C}$ & 109.5 \\
\hline Si1 & C19 & H19A & 109.5 & Si1 & C19 & H19B & 109.5 \\
\hline H19A & C19 & H19B & 109.5 & Si1 & C19 & $\mathrm{H} 19 \mathrm{C}$ & 109.5 \\
\hline H19A & C19 & $\mathrm{H} 19 \mathrm{C}$ & 109.5 & $\mathrm{H} 19 \mathrm{~B}$ & C19 & $\mathrm{H} 19 \mathrm{C}$ & 109.5 \\
\hline Si1 & $\mathrm{C} 20$ & $\mathrm{H} 20 \mathrm{~A}$ & 109.5 & Si1 & $\mathrm{C} 20$ & H2 OB & 109.5 \\
\hline $\mathrm{H} 20 \mathrm{~A}$ & C2 0 & $\mathrm{H} 20 \mathrm{~B}$ & 109.5 & Si1 & $\mathrm{C} 20$ & $\mathrm{H} 20 \mathrm{C}$ & 109.5 \\
\hline $\mathrm{H} 20 \mathrm{~A}$ & C2 0 & $\mathrm{H} 20 \mathrm{C}$ & 109.5 & $\mathrm{H} 2 \mathrm{OB}$ & $\mathrm{C} 20$ & $\mathrm{H} 20 \mathrm{C}$ & 109.5 \\
\hline C26 & C21 & C22 & $118.5(4)$ & C26 & C21 & $\mathrm{C} 2$ & $118.8(4)$ \\
\hline C22 & C21 & C2 & $122.7(4)$ & C23 & $\mathrm{C} 22$ & C21 & $120.0(5)$ \\
\hline $\mathrm{C} 23$ & $\mathrm{C} 22$ & H2 2A & 120.0 & $\mathrm{C} 21$ & $\mathrm{C} 22$ & H2 2A & 120.0 \\
\hline C22 & C23 & C24 & $120.4(5)$ & $\mathrm{C} 22$ & C23 & H2 3A & 119.8 \\
\hline C24 & C23 & H23A & 119.8 & C25 & C24 & C23 & $122.5(5)$ \\
\hline C25 & $\mathrm{C} 24$ & $\mathrm{H} 24 \mathrm{~A}$ & 118.8 & $\mathrm{C} 23$ & $\mathrm{C} 24$ & H2 4A & 118.8 \\
\hline C24 & C25 & $\mathrm{C} 26$ & $115.9(5)$ & $\mathrm{C} 24$ & $\mathrm{C} 25$ & H25A & 122.1 \\
\hline C26 & C25 & H25A & 122.1 & $\mathrm{C} 21$ & $\mathrm{C} 26$ & C25 & $122.6(5)$ \\
\hline C21 & $\mathrm{C} 26$ & $\mathrm{H} 26 \mathrm{~A}$ & 118.7 & C25 & $\mathrm{C} 26$ & H26A & 118.7 \\
\hline C32 & C27 & C2 8 & $117.7(4)$ & C32 & C27 & C3 & $123.0(4)$ \\
\hline C28 & C27 & C3 & $119.2(4)$ & C29 & $\mathrm{C} 28$ & C27 & $121.1(4)$ \\
\hline C29 & $\mathrm{C} 28$ & $\mathrm{H} 28 \mathrm{~A}$ & 119.4 & $\mathrm{C} 27$ & $\mathrm{C} 28$ & H2 $8 \mathrm{~A}$ & 119.4 \\
\hline C2 8 & C29 & C30 & $120.3(5)$ & C28 & C29 & H29A & 119.9 \\
\hline C30 & C29 & H29A & 119.9 & C31 & C30 & C29 & $119.3(5)$ \\
\hline C31 & C30 & $\mathrm{H} 30 \mathrm{~A}$ & 120.3 & C29 & C30 & $\mathrm{H} 30 \mathrm{~A}$ & 120.3 \\
\hline C30 & C31 & C32 & $121.0(5)$ & C30 & C31 & H31A & 119.5 \\
\hline C32 & C31 & H31A & 119.5 & C31 & C32 & C27 & $120.6(5)$ \\
\hline C31 & C32 & H32A & 119.7 & $\mathrm{C} 27$ & C32 & H32A & 119.7 \\
\hline Si2 & C33 & H33A & 109.5 & Si2 & C33 & H33 B & 109.5 \\
\hline H33A & C33 & H3 3 B & 109.5 & Si2 & C33 & H3 3C & 109.5 \\
\hline H3 3A & C33 & H3 3C & 109.5 & H3 3B & C33 & H3 3C & 109.5 \\
\hline Si2 & C34 & H3 4A & 109.5 & Si2 & C34 & H3 4B & 109.5 \\
\hline $\mathrm{H} 34 \mathrm{~A}$ & C34 & H3 4B & 109.5 & Si2 & C34 & H3 4C & 109.5 \\
\hline H34A & C34 & $\mathrm{H} 34 \mathrm{C}$ & 109.5 & H3 4B & C34 & H3 4C & 109.5 \\
\hline Si2 & C35 & H3 5 A & 109.5 & Si2 & C35 & H35B & 109.5 \\
\hline H35A & C35 & H3 5B & 109.5 & Si2 & C35 & H3 5C & 109.5 \\
\hline H3 5A & C35 & H35C & 109.5 & H3 5B & C35 & H3 5C & 109.5 \\
\hline C101 & $\mathrm{C} 100$ & C103\#1 & $72.4(8)$ & C101 & C100 & C104\# & $125.0(10)$ \\
\hline C103\#1 & $\mathrm{C} 100$ & C104\#1 & $52.6(7)$ & $\mathrm{C} 102$ & $\mathrm{C} 101$ & C100 & $175.6(12)$ \\
\hline C102 & C101 & $\mathrm{C} 103$ & $46.9(7)$ & C100 & C101 & $\mathrm{C} 103$ & $128.8(10)$ \\
\hline $\mathrm{C} 102$ & C101 & C103\#1 & - $117.8(11)$ & C100 & C101 & C103\#1 & $57.9(7)$ \\
\hline $\mathrm{C} 103$ & C101 & C103\#1 & $70.9(9)$ & C103 & C102 & C101 & $75.4(11)$ \\
\hline C103 & C102 & C104 & $59.5(10)$ & $\mathrm{C} 101$ & C102 & C104 & $134.9(12)$ \\
\hline $\mathrm{C} 102$ & C103 & C104 & $75.7(12)$ & C102 & $\mathrm{C} 103$ & C101 & $57.7(10)$ \\
\hline C104 & C103 & C101 & $133.4(13)$ & C102 & C103 & C100\#1 & $143.5(15)$ \\
\hline C104 & $\mathrm{C} 103$ & C100\#1 & $67.8(9)$ & C101 & $\mathrm{C} 103$ & C100\#1 & $158.8(12)$ \\
\hline C102 & C103 & C101\#1 & $166.8(15)$ & C104 & C103 & C101\#1 & $117.5(12)$ \\
\hline
\end{tabular}




$\begin{array}{llllllll}\mathrm{C} 101 & \mathrm{C} 103 & \mathrm{C} 101 \# 1 & 109.1(9) & \mathrm{C} 100 \# 1 & \mathrm{C} 103 & \mathrm{C} 101 \# 1 & 149.7(7) \\ \mathrm{C} 102 & \mathrm{C} 103 & \mathrm{C} 103 \# 1 & 117.0(17) & \mathrm{C} 104 & \mathrm{C} 103 & \mathrm{C} 103 \# 1 & 167.2(18) \\ \mathrm{C} 101 & \mathrm{C} 103 & \mathrm{C} 103 \# 1 & 59.3(9) & \mathrm{C} 100 \# 1 & \mathrm{C} 103 & \mathrm{C} 103 \# 1 & 99.4(13) \\ \mathrm{C} 101 \# 1 & \mathrm{C} 103 & \mathrm{C} 103 \# 1 & 49.8(7) & \mathrm{C} 103 & \mathrm{C} 104 & \mathrm{C} 102 & 44.8(7) \\ \mathrm{C} 103 & \mathrm{C} 104 & \mathrm{C} 100 \# 1 & 59.6(8) & \mathrm{C} 102 & \mathrm{C} 104 & \mathrm{C} 100 \# 1 & 104.4(8)\end{array}$

Table S10. Torsion angles $\left({ }^{\circ}\right)$ for 2.

\begin{tabular}{|c|c|c|c|c|c|c|c|c|c|}
\hline atom & atom & atom & atom & angle & atom & atom & atom & atom & angle \\
\hline C19 & Si1 & $\mathrm{C} 1$ & $\mathrm{C} 2$ & $-27.0(4)$ & C18 & Si1 & C1 & C2 & $-145.1(4)$ \\
\hline C20 & Si1 & $\mathrm{C} 1$ & $\mathrm{C} 2$ & $96.8(4)$ & C19 & Si1 & $\mathrm{C} 1$ & $\mathrm{Zr}$ & $158.3(3)$ \\
\hline C18 & Si1 & $\mathrm{C} 1$ & Zr & $40.2(3)$ & C20 & Si1 & $\mathrm{C} 1$ & Zr & $-77.9(3)$ \\
\hline C4 & Zr & $\mathrm{C} 1$ & $\mathrm{C} 2$ & $1.9(3)$ & C5 & Zr & $\mathrm{C} 1$ & $\mathrm{C} 2$ & $142.8(3)$ \\
\hline C13 & Zr & $\mathrm{C} 1$ & $\mathrm{C} 2$ & $-142.4(3)$ & C9 & Zr & $\mathrm{C} 1$ & $\mathrm{C} 2$ & $137.8(3)$ \\
\hline C14 & Zr & $\mathrm{C} 1$ & $\mathrm{C} 2$ & $-98.8(3)$ & C17 & Zr & $\mathrm{C} 1$ & $\mathrm{C} 2$ & $-138.0(3)$ \\
\hline $\mathrm{C} 6$ & $\mathrm{Zr}$ & $\mathrm{C} 1$ & $\mathrm{C} 2$ & $98.4(3)$ & $\mathrm{C} 8$ & Zr & $\mathrm{C} 1$ & $\mathrm{C} 2$ & $106.6(3)$ \\
\hline C15 & Zr & $\mathrm{C} 1$ & $\mathrm{C} 2$ & $-84.6(3)$ & C16 & Zr & $\mathrm{C} 1$ & $\mathrm{C} 2$ & $-106.9(3)$ \\
\hline C7 & Zr & $\mathrm{C} 1$ & $\mathrm{C} 2$ & $84.2(3)$ & C4 & Zr & $\mathrm{C} 1$ & Si1 & $177.5(3)$ \\
\hline C5 & Zr & $\mathrm{C} 1$ & Si1 & $-41.6(3)$ & C13 & Zr & $\mathrm{C} 1$ & Sil & $33.2(3)$ \\
\hline C9 & Zr & $\mathrm{C} 1$ & Si1 & $-46.6(3)$ & C14 & Zr & $\mathrm{C} 1$ & Si1 & $76.7(3)$ \\
\hline C17 & Zr & $\mathrm{C} 1$ & Si1 & $37.6(3)$ & C6 & $\mathrm{Zr}$ & $\mathrm{C} 1$ & Si1 & $-86.0(3)$ \\
\hline $\mathrm{C} 8$ & Zr & $\mathrm{C} 1$ & Si1 & $-77.8(3)$ & C15 & Zr & $\mathrm{C} 1$ & Sil & $90.9(3)$ \\
\hline C16 & Zr & $\mathrm{C} 1$ & Sil & $68.6(3)$ & C7 & Zr & $\mathrm{C} 1$ & Si1 & $-100.2(3)$ \\
\hline Si1 & $\mathrm{C} 1$ & $\mathrm{C} 2$ & $\mathrm{C} 21$ & $-4.4(6)$ & Zr & $\mathrm{C} 1$ & $\mathrm{C} 2$ & $\mathrm{C} 21$ & $171.7(3)$ \\
\hline Si1 & $\mathrm{C} 1$ & $\mathrm{C} 2$ & C3 & $177.8(3)$ & Zr & $\mathrm{C} 1$ & $\mathrm{C} 2$ & C3 & $-6.1(5)$ \\
\hline Si1 & $\mathrm{C} 1$ & $\mathrm{C} 2$ & Zr & $-176.1(4)$ & C4 & Zr & $\mathrm{C} 2$ & $\mathrm{C} 1$ & $-177.8(3)$ \\
\hline C5 & Zr & $\mathrm{C} 2$ & $\mathrm{C} 1$ & $-62.1(4)$ & C13 & Zr & $\mathrm{C} 2$ & $\mathrm{C} 1$ & $61.0(4)$ \\
\hline C9 & Zr & $\mathrm{C} 2$ & $\mathrm{C} 1$ & $-48.1(3)$ & C14 & Zr & $\mathrm{C} 2$ & $\mathrm{C} 1$ & $110.8(3)$ \\
\hline C17 & Zr & $\mathrm{C} 2$ & $\mathrm{C} 1$ & $47.5(3)$ & c6 & Zr & $\mathrm{C} 2$ & $\mathrm{C} 1$ & $-112.7(3)$ \\
\hline C8 & Zr & $\mathrm{C} 2$ & $\mathrm{C} 1$ & $-73.5(3)$ & C15 & $\mathrm{Zr}$ & $\mathrm{C} 2$ & $\mathrm{C} 1$ & $103.0(3)$ \\
\hline C16 & Zr & $\mathrm{C} 2$ & $\mathrm{C} 1$ & $72.3(3)$ & C7 & Zr & $\mathrm{C} 2$ & $\mathrm{C} 1$ & $-104.1(3)$ \\
\hline C4 & Zr & $\mathrm{C} 2$ & $\mathrm{C} 21$ & $137.0(17)$ & $\mathrm{C} 1$ & $\mathrm{Zr}$ & $\mathrm{C} 2$ & $\mathrm{C} 21$ & $-45.2(16)$ \\
\hline C5 & Zr & $\mathrm{C} 2$ & C21 & $-107.3(16)$ & C13 & $\mathrm{Zr}$ & $\mathrm{C} 2$ & $\mathrm{C} 21$ & $15.8(17)$ \\
\hline C9 & Zr & $\mathrm{C} 2$ & $\mathrm{C} 21$ & $-93.3(16)$ & C14 & Zr & $\mathrm{C} 2$ & $\mathrm{C} 21$ & $65.6(17)$ \\
\hline C17 & Zr & $\mathrm{C} 2$ & $\mathrm{C} 21$ & $2.3(17)$ & c6 & Zr & $\mathrm{C} 2$ & $\mathrm{C} 21$ & $-157.9(16)$ \\
\hline C8 & Zr & $\mathrm{C} 2$ & $\mathrm{C} 21$ & $-118.7(16)$ & C15 & $\mathrm{Zr}$ & $\mathrm{C} 2$ & $\mathrm{C} 21$ & $57.8(16)$ \\
\hline C16 & Zr & $\mathrm{C} 2$ & C21 & $27.2(16)$ & C7 & Zr & $\mathrm{C} 2$ & $\mathrm{C} 21$ & $-149.3(16)$ \\
\hline C4 & Zr & $\mathrm{C} 2$ & C3 & $-2.9(2)$ & $\mathrm{C} 1$ & Zr & $\mathrm{C} 2$ & C3 & $174.8(4)$ \\
\hline C5 & Zr & $\mathrm{C} 2$ & C3 & $112.7(3)$ & C13 & $\mathrm{Zr}$ & $\mathrm{C} 2$ & C3 & $-124.1(3)$ \\
\hline C9 & Zr & $\mathrm{C} 2$ & C3 & $126.8(2)$ & C14 & Zr & $\mathrm{C} 2$ & C3 & $-74.3(3)$ \\
\hline C17 & Zr & $\mathrm{C} 2$ & C3 & $-137.7(2)$ & c6 & Zr & $\mathrm{C} 2$ & C3 & $62.1(3)$ \\
\hline C8 & Zr & $\mathrm{C} 2$ & $\mathrm{C} 3$ & $101.4(2)$ & C15 & $\operatorname{Zr}$ & $\mathrm{C} 2$ & $\mathrm{C} 3$ & $-82.2(2)$ \\
\hline C16 & Zr & $\mathrm{C} 2$ & C3 & $-112.8(2)$ & C7 & Zr & $\mathrm{C} 2$ & C3 & $70.8(2)$ \\
\hline C1 & $\mathrm{C} 2$ & C3 & C4 & $10.1(7)$ & $\mathrm{C} 21$ & $\mathrm{C} 2$ & C3 & C4 & $-167.8(4)$ \\
\hline Zr & $\mathrm{C} 2$ & C3 & C4 & $5.1(4)$ & C1 & $\mathrm{C} 2$ & C3 & $\mathrm{C} 27$ & $-167.7(4)$ \\
\hline C21 & $\mathrm{C} 2$ & $\mathrm{C} 3$ & $\mathrm{C} 27$ & $14.4(5)$ & Zr & $\mathrm{C} 2$ & C3 & $\mathrm{C} 27$ & $-172.7(3)$ \\
\hline C1 & $\mathrm{C} 2$ & C3 & Zr & $5.0(4)$ & C21 & $\mathrm{C} 2$ & C3 & Zr & $-172.9(3)$ \\
\hline C1 & Zr & $\mathrm{C} 3$ & $\mathrm{C} 4$ & $-177.5(3)$ & C5 & Zr & $\mathrm{C} 3$ & $\mathrm{C} 4$ & $66.3(4)$ \\
\hline $\mathrm{C} 13$ & Zr & $\mathrm{C} 3$ & C4 & $-63.9(4)$ & C9 & Zr & C3 & C4 & $112.6(3)$ \\
\hline C14 & Zr & $\mathrm{C} 3$ & C4 & $-47.1(3)$ & C17 & $\mathrm{Zr}$ & C3 & C4 & $-114.2(3)$ \\
\hline C6 & Zr & $\mathrm{C} 3$ & $\mathrm{C} 4$ & $48.9(3)$ & $\mathrm{C} 8$ & Zr & C3 & C4 & $102.7(3)$ \\
\hline C15 & Zr & C3 & C4 & $-71.4(3)$ & C16 & $\mathrm{Zr}$ & C3 & C4 & $-102.9(3)$ \\
\hline C7 & Zr & C3 & C4 & $71.9(3)$ & C4 & $\mathrm{Zr}$ & C3 & $\mathrm{C} 27$ & $-43.0(15)$ \\
\hline $\mathrm{C} 1$ & Zr & C3 & C27 & $139.5(16)$ & C5 & $\operatorname{Zr}$ & C3 & $\mathrm{C} 27$ & $23.3(17)$ \\
\hline C13 & $\mathrm{Zr}$ & $\mathrm{C} 3$ & $\mathrm{C} 27$ & $-106.9(15)$ & C9 & Zr & $\mathrm{C} 3$ & $\mathrm{C} 27$ & $69.6(16)$ \\
\hline C14 & Zr & C3 & C27 & $-90.2(16)$ & C17 & $\mathrm{Zr}$ & C3 & $\mathrm{C} 27$ & $-157.2(15)$ \\
\hline
\end{tabular}




\begin{tabular}{|c|c|c|c|c|c|c|c|c|c|}
\hline C6 & Zr & $\mathrm{C} 3$ & $\mathrm{C} 27$ & $5.9(16)$ & $\mathrm{C} 8$ & $\mathrm{Zr}$ & C3 & $\mathrm{C} 27$ & $59.7(16)$ \\
\hline C15 & $\mathrm{Zr}$ & C3 & $\mathrm{C} 27$ & $-114.5(16)$ & C16 & $\mathrm{Zr}$ & $\mathrm{C} 3$ & $\mathrm{C} 27$ & $-145.9(15)$ \\
\hline C7 & Zr & C3 & $\mathrm{C} 27$ & $28.9(16)$ & C4 & Zr & C3 & C2 & $174.7(4)$ \\
\hline $\mathrm{C} 1$ & Zr & $\mathrm{C} 3$ & $\mathrm{C} 2$ & $-2.8(2)$ & C5 & Zr & $\mathrm{C} 3$ & $\mathrm{C} 2$ & $-119.0(3)$ \\
\hline $\mathrm{C} 13$ & Zr & C3 & $\mathrm{C} 2$ & $110.8(3)$ & C9 & Zr & C3 & $\mathrm{C} 2$ & $-72.7(3)$ \\
\hline C14 & Zr & C3 & $\mathrm{C} 2$ & $127.6(2)$ & C17 & Zr & C3 & $\mathrm{C} 2$ & $60.5(3)$ \\
\hline C6 & Zr & $\mathrm{C} 3$ & $\mathrm{C} 2$ & $-136.4(2)$ & $\mathrm{C} 8$ & Zr & C3 & $\mathrm{C} 2$ & $-82.6(2)$ \\
\hline C15 & Zr & C3 & $\mathrm{C} 2$ & $103.3(2)$ & $\mathrm{C} 16$ & Zr & $\mathrm{C} 3$ & $\mathrm{C} 2$ & $71.8(2)$ \\
\hline C7 & Zr & C3 & $\mathrm{C} 2$ & $-113.4(2)$ & C27 & C3 & C4 & Si2 & $-2.1(6)$ \\
\hline C2 & C3 & $\mathrm{C} 4$ & Si2 & $-179.7(3)$ & Zr & C3 & $\mathrm{C} 4$ & Si2 & $-173.4(4)$ \\
\hline C27 & C3 & C4 & $\mathrm{Zr}$ & $171.4(3)$ & $\mathrm{C} 2$ & C3 & C4 & Zr & $-6.3(5)$ \\
\hline C35 & Si2 & C4 & C3 & $91.6(4)$ & C33 & Si2 & C4 & C3 & $-151.7(4)$ \\
\hline C34 & $\mathrm{Si2}$ & $\mathrm{C} 4$ & $\mathrm{C} 3$ & $-32.4(5)$ & C35 & $\mathrm{Si} 2$ & $\mathrm{C} 4$ & $\mathrm{Zr}$ & $-79.3(3)$ \\
\hline C33 & Si2 & C4 & $\mathrm{Zr}$ & $37.4(3)$ & C34 & Si2 & C4 & $\mathrm{Zr}$ & $156.7(3)$ \\
\hline C1 & Zr & C4 & C3 & $2.2(3)$ & C5 & Zr & C4 & $\mathrm{C} 3$ & $-139.2(3)$ \\
\hline C13 & Zr & $\mathrm{C} 4$ & C3 & $144.1(3)$ & C9 & Zr & C4 & $\mathrm{C} 3$ & $-95.1(3)$ \\
\hline C14 & Zr & C4 & C3 & $139.7(3)$ & C17 & Zr & C4 & C3 & $98.2(3)$ \\
\hline C6 & Zr & $\mathrm{C} 4$ & C3 & $-136.8(3)$ & $\mathrm{C} 8$ & Zr & C4 & C3 & $-83.6(3)$ \\
\hline C15 & Zr & C4 & C3 & $109.5(3)$ & C16 & Zr & C4 & C3 & $85.7(3)$ \\
\hline C7 & Zr & C4 & C3 & $-106.8(3)$ & $\mathrm{C} 1$ & Zr & C4 & Si2 & $174.6(3)$ \\
\hline C5 & Zr & C4 & Si2 & $33.3(4)$ & $\mathrm{C} 13$ & Zr & C4 & Si2 & $-43.5(4)$ \\
\hline C9 & Zr & C4 & Si2 & $77.3(4)$ & C14 & Zr & C4 & Si2 & $-47.8(3)$ \\
\hline C17 & Zr & C4 & Si2 & $-89.3(3)$ & $\mathrm{C} 6$ & Zr & C4 & Si2 & $35.6(3)$ \\
\hline C8 & Zr & C4 & Si2 & $88.9(3)$ & C15 & Zr & C4 & Si2 & $-78.0(3)$ \\
\hline C16 & Zr & C4 & Si2 & $-101.8(3)$ & C7 & Zr & C4 & Si2 & $65.7(3)$ \\
\hline C4 & Zr & C5 & $\mathrm{C} 6$ & $4.3(3)$ & $\mathrm{C} 1$ & Zr & C5 & $\mathrm{C} 6$ & $-122.4(3)$ \\
\hline C13 & Zr & C5 & C6 & $123.7(3)$ & C9 & Zr & C5 & C6 & $-113.3(4)$ \\
\hline C14 & Zr & C5 & C6 & $95.3(3)$ & C17 & Zr & C5 & C6 & $150.6(3)$ \\
\hline C8 & Zr & C5 & C6 & $-75.9(3)$ & C15 & Zr & C5 & C6 & $105.5(3)$ \\
\hline C16 & Zr & C5 & C6 & $139.2(3)$ & C7 & Zr & C5 & C6 & $-36.7(2)$ \\
\hline C4 & Zr & C5 & C9 & $117.6(3)$ & $\mathrm{C} 1$ & Zr & C5 & C9 & $-9.1(3)$ \\
\hline C13 & Zr & C5 & C9 & $-123.0(3)$ & C14 & Zr & C5 & C9 & $-151.4(3)$ \\
\hline C17 & Zr & C5 & C9 & $-96.1(3)$ & C6 & Zr & C5 & C9 & $113.3(4)$ \\
\hline C8 & Zr & C5 & C9 & $37.5(2)$ & C15 & Zr & C5 & C9 & $-141.2(3)$ \\
\hline C16 & Zr & C5 & C9 & $-107.5(3)$ & C7 & Zr & C5 & C9 & $76.7(3)$ \\
\hline C4 & Zr & C5 & C10 & $-119.7(3)$ & $\mathrm{C} 1$ & $\mathrm{Zr}$ & C5 & C10 & $113.6(3)$ \\
\hline C13 & Zr & C5 & C10 & $-0.3(3)$ & C9 & $\mathrm{Zr}$ & C5 & C10 & $122.7(4)$ \\
\hline C14 & Zr & C5 & C10 & $-28.7(3)$ & C17 & Zr & C5 & $\mathrm{C} 10$ & $26.6(3)$ \\
\hline C6 & Zr & C5 & C10 & $-124.0(4)$ & $\mathrm{C} 8$ & Zr & C5 & C10 & $160.1(4)$ \\
\hline C15 & Zr & C5 & C10 & $-18.5(3)$ & C16 & Zr & C5 & $\mathrm{C} 10$ & $15.2(3)$ \\
\hline C7 & Zr & C5 & C10 & $-160.7(4)$ & C9 & C5 & C6 & C7 & $1.4(5)$ \\
\hline C10 & C5 & C6 & C7 & $161.8(4)$ & Zr & C5 & C6 & C7 & $68.7(3)$ \\
\hline C9 & C5 & C6 & Zr & $-67.4(3)$ & C10 & C5 & C6 & $\mathrm{Zr}$ & $93.1(4)$ \\
\hline C4 & Zr & C6 & C5 & $-176.6(3)$ & C1 & Zr & C6 & C5 & $89.3(3)$ \\
\hline $\mathrm{C} 13$ & Zr & C6 & C5 & $-45.5(3)$ & C9 & Zr & C6 & C5 & $38.3(2)$ \\
\hline C14 & Zr & C6 & C5 & $-79.0(3)$ & C17 & Zr & C6 & C5 & $-30.3(3)$ \\
\hline C8 & Zr & C6 & C5 & $79.0(3)$ & C15 & Zr & C6 & C5 & $-87.6(3)$ \\
\hline C16 & Zr & C6 & C5 & $-55.9(3)$ & $\mathrm{C} 7$ & $\mathrm{Zr}$ & $\mathrm{C} 6$ & C5 & $115.0(4)$ \\
\hline C4 & Zr & C6 & C7 & $68.3(3)$ & $\mathrm{C} 1$ & Zr & C6 & C7 & $-25.8(4)$ \\
\hline C5 & Zr & C6 & C7 & $-115.0(4)$ & C13 & Zr & C6 & C7 & $-160.6(3)$ \\
\hline C9 & Zr & C6 & C7 & $-76.8(3)$ & C14 & Zr & C6 & $\mathrm{C} 7$ & $165.9(3)$ \\
\hline C17 & Zr & C6 & C7 & $-145.3(3)$ & C8 & Zr & C6 & C7 & $-36.1(2)$ \\
\hline C15 & Zr & C6 & C7 & $157.4(3)$ & C16 & Zr & C6 & C7 & $-170.9(2)$ \\
\hline C5 & C6 & C7 & C8 & $-1.5(5)$ & $\mathrm{Zr}$ & C6 & C7 & C8 & $64.4(3)$ \\
\hline C5 & C6 & C7 & $\mathrm{Zr}$ & $-65.9(3)$ & C4 & Zr & C7 & C8 & $133.1(3)$ \\
\hline C1 & Zr & C7 & C8 & $46.7(3)$ & C5 & Zr & C7 & $\mathrm{C} 8$ & $-78.2(3)$ \\
\hline C13 & Zr & C7 & C8 & $-95.6(3)$ & C9 & Zr & C7 & $\mathrm{C} 8$ & $-36.8(3)$ \\
\hline
\end{tabular}




\begin{tabular}{|c|c|c|c|c|c|c|c|c|c|}
\hline C14 & Zr & C7 & C8 & $-131.0(3)$ & C17 & Zr & C7 & C8 & $-68.5(3)$ \\
\hline C6 & Zr & C7 & $\mathrm{C} 8$ & $-115.7(4)$ & C15 & $\mathrm{Zr}$ & C7 & C8 & $-150.9(3)$ \\
\hline C16 & Zr & C7 & C8 & $-91.9(6)$ & C4 & Zr & C7 & C6 & $-111.2(3)$ \\
\hline $\mathrm{C} 1$ & Zr & $\mathrm{C7}$ & $\mathrm{C} 6$ & $162.4(3)$ & C5 & Zr & C7 & C6 & $37.5(2)$ \\
\hline $\mathrm{C} 13$ & Zr & C7 & C6 & $20.2(3)$ & C9 & Zr & C7 & C6 & $78.9(3)$ \\
\hline C14 & Zr & C7 & C6 & $-15.3(3)$ & C17 & Zr & C7 & C6 & $47.2(3)$ \\
\hline $\mathrm{C} 8$ & $\mathrm{Zr}$ & C7 & C6 & $115.7(4)$ & C15 & Zr & C7 & C6 & $-35.2(4)$ \\
\hline C16 & Zr & C7 & C6 & $23.9(7)$ & $\mathrm{C} 6$ & C7 & $\mathrm{C} 8$ & C9 & $1.1(5)$ \\
\hline $\mathrm{Zr}$ & C7 & $\mathrm{C} 8$ & C9 & $64.2(3)$ & C6 & C7 & C8 & Zr & $-63.1(3)$ \\
\hline C4 & Zr & $\mathrm{C} 8$ & C7 & $-48.5(3)$ & C1 & Zr & $\mathrm{C} 8$ & C7 & $-136.3(3)$ \\
\hline C5 & Zr & C8 & C7 & $78.2(3)$ & C13 & Zr & C8 & C7 & $95.5(3)$ \\
\hline C9 & Zr & C8 & C7 & $116.4(4)$ & C14 & Zr & C8 & C7 & $66.7(3)$ \\
\hline C17 & Zr & $\mathrm{C} 8$ & C7 & $130.3(3)$ & C6 & Zr & $\mathrm{C} 8$ & C7 & $36.9(3)$ \\
\hline C15 & Zr & $\mathrm{C} 8$ & C7 & $82.5(6)$ & C16 & Zr & $\mathrm{C} 8$ & C7 & $149.5(3)$ \\
\hline C4 & Zr & C8 & C9 & $-164.9(3)$ & $\mathrm{C} 1$ & Zr & $\mathrm{C} 8$ & C9 & $107.3(3)$ \\
\hline C5 & Zr & $\mathrm{C} 8$ & C9 & $-38.2(3)$ & C13 & Zr & $\mathrm{C} 8$ & C9 & $-20.9(3)$ \\
\hline C14 & Zr & $\mathrm{C} 8$ & C9 & $-49.7(4)$ & C17 & Zr & $\mathrm{C} 8$ & C9 & $13.9(3)$ \\
\hline C6 & Zr & C8 & C9 & $-79.5(3)$ & C15 & Zr & $\mathrm{C} 8$ & C9 & $-33.9(7)$ \\
\hline C16 & Zr & $\mathrm{C} 8$ & C9 & $33.1(4)$ & C7 & Zr & C8 & C9 & $-116.4(4)$ \\
\hline C7 & $\mathrm{C} 8$ & C9 & C5 & $-0.3(5)$ & Zr & C8 & C9 & C5 & $66.5(3)$ \\
\hline C7 & C8 & C9 & Zr & $-66.7(3)$ & $\mathrm{C} 6$ & C5 & C9 & $\mathrm{C} 8$ & $-0.7(5)$ \\
\hline C10 & C5 & C9 & $\mathrm{C} 8$ & $-161.4(4)$ & Zr & C5 & C9 & $\mathrm{C} 8$ & $-68.4(3)$ \\
\hline C6 & C5 & C9 & Zr & $67.8(3)$ & C10 & C5 & C9 & Zr & $-93.0(4)$ \\
\hline C4 & Zr & C9 & $\mathrm{C} 8$ & $21.1(4)$ & $\mathrm{C} 1$ & Zr & C9 & $\mathrm{C} 8$ & $-73.3(3)$ \\
\hline C5 & Zr & C9 & C8 & $113.8(4)$ & $\mathrm{C} 13$ & Zr & C9 & C8 & $159.9(3)$ \\
\hline C14 & Zr & C9 & C8 & $143.2(3)$ & C17 & Zr & C9 & $\mathrm{C} 8$ & $-167.5(3)$ \\
\hline C6 & Zr & C9 & $\mathrm{C} 8$ & $75.8(3)$ & C15 & Zr & C9 & C8 & $167.0(3)$ \\
\hline C16 & Zr & C9 & C8 & $-159.7(3)$ & C7 & Zr & C9 & $\mathrm{C} 8$ & $35.6(3)$ \\
\hline C4 & Zr & C9 & C5 & $-92.7(3)$ & $\mathrm{C} 1$ & Zr & C9 & C5 & $172.9(3)$ \\
\hline C13 & Zr & $\mathrm{C} 9$ & $\mathrm{C} 5$ & $46.0(3)$ & C14 & $\mathrm{Zr}$ & C9 & $\mathrm{C} 5$ & $29.4(3)$ \\
\hline C17 & Zr & C9 & $\mathrm{C} 5$ & $78.7(3)$ & $\mathrm{C} 6$ & Zr & C9 & $\mathrm{C} 5$ & $-38.0(2)$ \\
\hline C8 & Zr & C9 & C5 & $-113.8(4)$ & C15 & Zr & C9 & C5 & $53.2(3)$ \\
\hline C16 & Zr & C9 & C5 & $86.4(3)$ & C7 & Zr & C9 & C5 & $-78.2(3)$ \\
\hline C6 & C5 & C10 & C12 & $40.4(6)$ & C9 & C5 & C10 & C12 & $-162.3(4)$ \\
\hline Zr & C5 & C10 & C12 & $119.5(3)$ & C6 & C5 & C10 & C13 & $-78.6(5)$ \\
\hline C9 & C5 & C10 & C13 & $78.6(5)$ & Zr & C5 & $\mathrm{C} 10$ & C13 & $0.4(4)$ \\
\hline C6 & C5 & C10 & C11 & $163.5(4)$ & C9 & C5 & C10 & C11 & $-39.3(6)$ \\
\hline Zr & C5 & C10 & C11 & $-117.5(3)$ & $\mathrm{C} 12$ & $\mathrm{C} 10$ & C13 & C17 & $162.8(4)$ \\
\hline C5 & C10 & C13 & C17 & $-79.1(5)$ & C11 & $\mathrm{C} 10$ & C13 & C17 & $38.7(6)$ \\
\hline C12 & $\mathrm{C} 10$ & C13 & C14 & $-40.4(6)$ & C5 & $\mathrm{C} 10$ & C13 & C14 & $77.7(5)$ \\
\hline C11 & C10 & C13 & C14 & $-164.5(4)$ & C12 & C10 & C13 & Zr & $-118.5(3)$ \\
\hline C5 & C10 & C13 & Zr & $-0.4(4)$ & C11 & C10 & C13 & Zr & $117.5(3)$ \\
\hline C4 & Zr & C13 & C17 & $-121.0(3)$ & $\mathrm{C} 1$ & Zr & C13 & C17 & $8.2(3)$ \\
\hline C5 & Zr & C13 & C17 & $124.8(3)$ & C9 & Zr & C13 & C17 & $96.8(3)$ \\
\hline C14 & Zr & C13 & C17 & $-113.2(4)$ & C6 & Zr & C13 & C17 & $152.4(3)$ \\
\hline C8 & Zr & C13 & C17 & $108.1(3)$ & C15 & Zr & C13 & C17 & $-76.6(3)$ \\
\hline C16 & Zr & $\mathrm{C} 13$ & C17 & $-37.1(2)$ & C7 & Zr & C13 & C17 & $141.6(2)$ \\
\hline C4 & Zr & C13 & C14 & $-7.8(3)$ & $\mathrm{C} 1$ & Zr & C13 & C14 & $121.4(3)$ \\
\hline C5 & Zr & C13 & C14 & $-122.0(3)$ & C9 & Zr & C13 & C14 & $-150.0(3)$ \\
\hline C17 & Zr & C13 & C14 & $113.2(4)$ & C6 & Zr & C13 & C14 & $-94.4(3)$ \\
\hline C8 & Zr & C13 & C14 & $-138.7(3)$ & C15 & Zr & C13 & C14 & $36.5(2)$ \\
\hline C16 & Zr & C13 & C14 & $76.0(3)$ & C7 & Zr & C13 & C14 & $-105.3(3)$ \\
\hline $\mathrm{C} 4$ & Zr & $\mathrm{C} 13$ & $\mathrm{C} 10$ & $114.5(3)$ & $\mathrm{C} 1$ & Zr & C13 & $\mathrm{C} 10$ & $-116.3(3)$ \\
\hline C5 & Zr & C13 & C10 & $0.3(3)$ & C9 & Zr & C13 & C10 & $-27.7(3)$ \\
\hline C14 & Zr & C13 & C10 & $122.3(4)$ & C17 & Zr & C13 & $\mathrm{C} 10$ & $-124.5(4)$ \\
\hline C6 & Zr & C13 & C10 & $27.9(3)$ & C8 & Zr & C13 & C10 & $-16.4(3)$ \\
\hline C15 & Zr & C13 & C10 & $158.8(3)$ & C16 & Zr & C13 & C10 & $-161.6(3)$ \\
\hline
\end{tabular}




\begin{tabular}{|c|c|c|c|c|c|c|c|c|c|}
\hline C7 & Zr & C13 & $\mathrm{C} 10$ & $17.0(3)$ & C17 & $\mathrm{C} 13$ & C14 & C15 & $-0.9(5)$ \\
\hline C10 & C13 & C14 & C15 & $-161.4(4)$ & Zr & C13 & C14 & C15 & $-68.5(3)$ \\
\hline C17 & $\mathrm{C} 13$ & C14 & Zr & $67.6(3)$ & $\mathrm{C} 10$ & C13 & $\mathrm{C} 14$ & Zr & $-92.9(4)$ \\
\hline C4 & Zr & C14 & C15 & $-71.0(3)$ & $\mathrm{C} 1$ & Zr & C14 & C15 & $26.1(4)$ \\
\hline C5 & $\mathrm{Zr}$ & C14 & C15 & $161.6(3)$ & $\mathrm{C} 13$ & Zr & C14 & C15 & $114.9(4)$ \\
\hline C9 & $\mathrm{Zr}$ & C14 & C15 & $145.7(3)$ & C17 & Zr & C14 & C15 & $77.0(3)$ \\
\hline C6 & Zr & C14 & C15 & $-165.2(3)$ & C8 & Zr & C14 & C15 & $171.2(3)$ \\
\hline C16 & Zr & C14 & C15 & $36.2(2)$ & C7 & Zr & C14 & C15 & $-157.0(3)$ \\
\hline C4 & Zr & C14 & C13 & $174.1(3)$ & $\mathrm{C} 1$ & Zr & C14 & C13 & $-88.8(3)$ \\
\hline C5 & Zr & C14 & C13 & $46.8(3)$ & C9 & Zr & C14 & $\mathrm{C} 13$ & $30.8(3)$ \\
\hline C17 & Zr & C14 & C13 & $-37.8(2)$ & C6 & Zr & C14 & $\mathrm{C} 13$ & $79.9(3)$ \\
\hline $\mathrm{C} 8$ & Zr & C14 & C13 & $56.3(3)$ & C15 & Zr & C14 & C13 & $-114.9(4)$ \\
\hline C16 & $\mathrm{Zr}$ & C14 & C13 & $-78.7(3)$ & C7 & $\mathrm{Zr}$ & C14 & $\mathrm{C} 13$ & $88.1(3)$ \\
\hline C13 & C14 & C15 & C16 & $0.6(5)$ & $\mathrm{Zr}$ & C14 & C15 & C16 & $-65.6(3)$ \\
\hline C13 & C14 & C15 & Zr & $66.2(3)$ & C4 & Zr & C15 & $\mathrm{C} 14$ & $110.4(3)$ \\
\hline C1 & Zr & C15 & C14 & $-161.8(3)$ & C5 & Zr & C15 & C14 & $-19.0(3)$ \\
\hline C13 & Zr & C15 & C14 & $-37.9(2)$ & C9 & Zr & C15 & C14 & $-46.5(3)$ \\
\hline C17 & Zr & C15 & C14 & $-78.7(3)$ & C6 & $\operatorname{Zr}$ & C15 & C14 & $16.1(3)$ \\
\hline C8 & Zr & C15 & C14 & $-22.6(7)$ & $\mathrm{C} 16$ & Zr & C15 & C14 & $-116.0(4)$ \\
\hline C7 & Zr & C15 & C14 & $35.8(4)$ & C4 & Zr & C15 & C16 & $-133.6(3)$ \\
\hline $\mathrm{C} 1$ & Zr & C15 & C16 & $-45.7(3)$ & C5 & Zr & C15 & C16 & $97.1(3)$ \\
\hline C13 & Zr & C15 & C16 & $78.2(3)$ & C9 & Zr & C15 & C16 & $69.5(3)$ \\
\hline C14 & Zr & C15 & C16 & $116.0(4)$ & C17 & Zr & C15 & $\mathrm{C} 16$ & $37.4(2)$ \\
\hline C6 & Zr & C15 & C16 & $132.1(3)$ & C8 & $\mathrm{Zr}$ & C15 & C16 & $93.4(6)$ \\
\hline C7 & Zr & C15 & C16 & $151.8(3)$ & C14 & C15 & C16 & C17 & $-0.1(5)$ \\
\hline Zr & C15 & C16 & C17 & $-64.0(3)$ & C14 & C15 & C16 & Zr & $63.9(3)$ \\
\hline C4 & Zr & C16 & C15 & $49.8(3)$ & $\mathrm{C} 1$ & Zr & C16 & C15 & $136.6(3)$ \\
\hline C5 & Zr & C16 & C15 & $-94.2(3)$ & $\mathrm{C} 13$ & $\mathrm{Zr}$ & C16 & C15 & $-78.1(3)$ \\
\hline C9 & Zr & C16 & C15 & $-129.6(3)$ & C14 & $\operatorname{Zr}$ & C16 & C15 & $-36.6(2)$ \\
\hline C17 & Zr & C16 & C15 & $-115.3(4)$ & $\mathrm{C} 6$ & $\mathrm{Zr}$ & C16 & C15 & $-65.6(3)$ \\
\hline $\mathrm{C} 8$ & Zr & C16 & C15 & $-148.6(3)$ & C7 & Zr & C16 & C15 & $-82.4(6)$ \\
\hline C4 & Zr & C16 & C17 & $165.0(3)$ & $\mathrm{C} 1$ & Zr & C16 & C17 & $-108.1(3)$ \\
\hline C5 & Zr & C16 & C17 & $21.1(3)$ & $\mathrm{C} 13$ & Zr & C16 & C17 & $37.1(3)$ \\
\hline C9 & Zr & C16 & C17 & $-14.3(3)$ & C14 & Zr & C16 & $\mathrm{C} 17$ & $78.7(3)$ \\
\hline C6 & Zr & C16 & C17 & $49.7(3)$ & C8 & Zr & C16 & C17 & $-33.3(4)$ \\
\hline C15 & Zr & C16 & C17 & $115.3(4)$ & C7 & Zr & C16 & C17 & $32.8(7)$ \\
\hline C14 & C13 & C17 & C16 & $0.8(5)$ & C10 & $\mathrm{C} 13$ & $\mathrm{C} 17$ & C16 & $160.8(4)$ \\
\hline Zr & C13 & C17 & C16 & $68.1(3)$ & C14 & C13 & C17 & Zr & $-67.3(3)$ \\
\hline $\mathrm{C} 10$ & C13 & C17 & Zr & $92.7(4)$ & C15 & C16 & C17 & $\mathrm{C} 13$ & $-0.5(5)$ \\
\hline Zr & C16 & C17 & $\mathrm{C} 13$ & $-66.2(3)$ & C15 & C16 & C17 & Zr & $65.7(3)$ \\
\hline C4 & Zr & C17 & $\mathrm{C} 13$ & $92.7(3)$ & $\mathrm{C} 1$ & Zr & C17 & $\mathrm{C} 13$ & $-173.4(3)$ \\
\hline C5 & Zr & C17 & C13 & $-44.7(3)$ & C9 & $\mathrm{Zr}$ & C17 & C13 & $-78.0(3)$ \\
\hline C14 & Zr & C17 & C13 & $38.5(2)$ & C6 & $\mathrm{Zr}$ & C17 & $\mathrm{C} 13$ & $-28.4(3)$ \\
\hline C8 & Zr & C17 & $\mathrm{C} 13$ & $-85.4(3)$ & C15 & $\mathrm{Zr}$ & C17 & $\mathrm{C} 13$ & $78.7(3)$ \\
\hline C16 & Zr & C17 & C13 & $115.0(4)$ & C7 & $\operatorname{Zr}$ & C17 & C13 & $-52.7(3)$ \\
\hline C4 & Zr & C17 & C16 & $-22.2(4)$ & $\mathrm{C} 1$ & $\mathrm{Zr}$ & C17 & $\mathrm{C} 16$ & $71.6(3)$ \\
\hline C5 & Zr & C17 & C16 & $-159.7(3)$ & $\mathrm{C} 13$ & $\mathrm{Zr}$ & C17 & C16 & $-115.0(4)$ \\
\hline C9 & Zr & C17 & C16 & $167.1(3)$ & C14 & $\mathrm{Zr}$ & C17 & C16 & $-76.4(3)$ \\
\hline C6 & Zr & C17 & C16 & $-143.4(3)$ & C8 & $\mathrm{Zr}$ & C17 & C16 & $159.6(3)$ \\
\hline C15 & Zr & C17 & $\mathrm{C} 16$ & $-36.3(3)$ & C7 & $\mathrm{Zr}$ & C17 & C16 & $-167.7(3)$ \\
\hline C1 & $\mathrm{C} 2$ & C21 & $\mathrm{C} 26$ & $-74.6(5)$ & C3 & $\mathrm{C} 2$ & $\mathrm{C} 21$ & $\mathrm{C} 26$ & $103.4(4)$ \\
\hline $\mathrm{Zr}$ & $\mathrm{C} 2$ & C21 & C2 6 & $-34.1(18)$ & $\mathrm{C} 1$ & $\mathrm{C} 2$ & C21 & $C 22$ & $105.4(5)$ \\
\hline C3 & $\mathrm{C} 2$ & $\mathrm{C} 21$ & $\mathrm{C} 22$ & $-76.5(5)$ & $\mathrm{Zr}$ & $\mathrm{C} 2$ & $\mathrm{C} 21$ & $\mathrm{C} 22$ & $145.9(14)$ \\
\hline C26 & C21 & C22 & $\mathrm{C} 23$ & $0.0(7)$ & C2 & C21 & $\mathrm{C} 22$ & C23 & $179.9(4)$ \\
\hline C21 & C22 & C23 & $\mathrm{C} 24$ & $-1.9(7)$ & $\mathrm{C} 22$ & $\mathrm{C} 23$ & $\mathrm{C} 24$ & C25 & $0.7(8)$ \\
\hline C23 & C24 & C25 & $\mathrm{C} 26$ & $2.2(7)$ & C22 & $\mathrm{C} 21$ & C26 & C25 & $3.2(7)$ \\
\hline $\mathrm{C} 2$ & $\mathrm{C} 21$ & C26 & C2 5 & $-176.7(4)$ & C24 & C25 & $\mathrm{C} 26$ & $\mathrm{C} 21$ & $-4.2(7)$ \\
\hline
\end{tabular}




\begin{tabular}{|c|c|c|c|c|c|c|c|c|c|}
\hline $\mathrm{C} 4$ & C3 & $\mathrm{C} 27$ & C32 & $100.4(5)$ & $\mathrm{C} 2$ & C3 & $\mathrm{C} 27$ & C32 & $-81.7(5)$ \\
\hline Zr & C3 & $\mathrm{C} 27$ & C32 & $138.5(14)$ & $\mathrm{C} 4$ & $\mathrm{C} 3$ & $\mathrm{C} 27$ & $\mathrm{C} 28$ & $-77.5(5)$ \\
\hline $\mathrm{C} 2$ & $\mathrm{C} 3$ & $\mathrm{C} 27$ & $\mathrm{C} 28$ & $100.4(5)$ & Zr & C3 & $\mathrm{C} 27$ & $\mathrm{C} 28$ & $-39.3(18)$ \\
\hline C32 & $\mathrm{C} 27$ & $\mathrm{C} 28$ & $\mathrm{C} 29$ & $-0.3(7)$ & $\mathrm{C} 3$ & $\mathrm{C} 27$ & $\mathrm{C} 28$ & $\mathrm{C} 29$ & $177.7(4)$ \\
\hline $\mathrm{C} 27$ & $\mathrm{C} 28$ & C29 & C30 & $-0.6(7)$ & $\mathrm{C} 28$ & C29 & C30 & C31 & $0.7(8)$ \\
\hline $\mathrm{C} 29$ & $\mathrm{C} 30$ & C31 & C32 & $0.1(8)$ & $\mathrm{C} 30$ & C31 & C32 & $\mathrm{C} 27$ & $-1.0(7)$ \\
\hline C2 8 & $\mathrm{C} 27$ & C32 & C31 & $1.0(7)$ & $\mathrm{C} 3$ & C2 7 & C32 & C31 & $-176.8(4)$ \\
\hline C103\#1 & $\mathrm{C} 100$ & C101 & $\mathrm{C} 102$ & $13(13)$ & C104\#1 & C100 & C101 & $\mathrm{C} 102$ & $14(14)$ \\
\hline C103\#1 & C100 & C101 & C103 & $0.4(15)$ & C104\#1 & C100 & C101 & C103 & $1.8(17)$ \\
\hline C104\#1 & $\mathrm{C} 100$ & C101 & c103\#1 & $1.3(8)$ & C100 & C101 & $\mathrm{C} 102$ & $\mathrm{C} 103$ & $-13(13)$ \\
\hline C103\#1 & C101 & C102 & C103 & $-1.0(16)$ & C100 & C101 & C102 & C104 & $-13(14)$ \\
\hline C103 & $\mathrm{C} 101$ & $\mathrm{C} 102$ & $\mathrm{C} 104$ & $0.4(11)$ & C103\#1 & C101 & $\mathrm{C} 102$ & $\mathrm{C} 104$ & $-0.6(19)$ \\
\hline C101 & $\mathrm{C} 102$ & C103 & C104 & $179.7(9)$ & C104 & C102 & C103 & C101 & $-179.7(9)$ \\
\hline C101 & $\mathrm{C} 102$ & C103 & c100\#1 & $180(2)$ & C104 & C102 & C103 & C100\#1 & $0(2)$ \\
\hline C101 & $\mathrm{C} 102$ & C103 & C101\#1 & $4(6)$ & C104 & C102 & C103 & C101\#1 & $-176(6)$ \\
\hline C101 & $\mathrm{C} 102$ & $\mathrm{C} 103$ & c103\#1 & $0.9(14)$ & C104 & $\mathrm{C} 102$ & $\mathrm{C} 103$ & C103\#1 & $-178.8(17)$ \\
\hline C100 & C101 & C103 & C102 & $178.7(13)$ & C103\#1 & C101 & C103 & $\mathrm{C} 102$ & $179.1(15)$ \\
\hline C102 & C101 & C103 & C104 & $-0.4(12)$ & C100 & C101 & C103 & C104 & $178.3(11)$ \\
\hline C103\#1 & C101 & C103 & C104 & $178.7(19)$ & C102 & C101 & C103 & C100\#1 & $-180(100)$ \\
\hline C100 & C101 & C103 & C100\#1 & $-1(4)$ & C103\#1 & C101 & C103 & C100\#1 & $-1(3)$ \\
\hline C102 & C101 & C103 & C101\#1 & $-179.1(15)$ & C100 & C101 & C103 & C101\#1 & $-0.4(14)$ \\
\hline C103\#1 & C101 & C103 & C101\#1 & $0.000(1)$ & C102 & C101 & C103 & C103\#1 & $-179.1(15)$ \\
\hline C100 & C101 & C103 & C103\#1 & $-0.4(14)$ & C101 & C103 & C104 & $\mathrm{C} 102$ & $0.4(11)$ \\
\hline C100\#1 & C103 & C104 & C102 & $-179.9(13)$ & C101\#1 & C103 & C104 & $\mathrm{C} 102$ & $178.9(16)$ \\
\hline C103\#1 & C103 & C104 & C102 & $175(7)$ & C102 & C103 & C104 & C100\#1 & $179.9(13)$ \\
\hline C101 & C103 & C104 & C100\#1 & $-179.8(17)$ & C101\#1 & C103 & C104 & C100\#1 & $-1.2(7)$ \\
\hline C103\#1 & C103 & C104 & C100\#1 & $-5(6)$ & C101 & C102 & C104 & C103 & $-0.4(13)$ \\
\hline C103 & C102 & C104 & C100\#1 & $-0.1(12)$ & C101 & C102 & C104 & C100\#1 & $-0.5(17)$ \\
\hline
\end{tabular}

\section{References}

(1) SAINT: SAX Area-Dectector Integration Program, V5.04; Siemens Industrial Automation, Inc.: Madison, WI, (1995)

(2) XPREP: (v 5.03) Part of the SHELXTL Crystal Structure Determination Package, Siemens Industrial Automation, Inc.: Madison, WI, (1995)

(3) SADABS: Siemens Area Detector ABSorption correction program, George Sheldrick, (1996). Advance copy, private communication.

(4) XS: Program for the Solution of X-ray Crystal Structures, Part of the SHELXTL Crystal Structure Determination Package, Bruker Analytical X-ray Systems Inc.: Madison, WI, (1995-99)

(5) XL: Program for the Refinement of X-ray Crystal Structures, Part of the SHELXTL Crystal Structure Determination Package, Bruker Analytical X-ray Systems Inc.: Madison, WI, (1995-99)

(6) Least-Squares: Function minimized $\Sigma w\left(\left|\mathrm{~F}_{\mathrm{o}}\right|^{2}-\left|\mathrm{F}_{\mathrm{c}}\right|^{2}\right)^{2}$

(7) Standard deviation of an observation of unit weight: $\left[\sum w\left(\left|\mathrm{~F}_{\mathrm{o}}\right|^{2}-\left|\mathrm{F}_{\mathrm{c}}\right|^{2}\right)^{2} /\left(\mathrm{N}_{\mathrm{o}}-\mathrm{N}_{\mathrm{v}}\right)\right]^{1 / 2}$ where $\mathrm{N}_{\mathrm{o}}=$ number of observations

$\mathrm{N}_{\mathrm{v}}=$ number of variables 
(8) Cromer, D. T. \& Waber, J. T.; "International Tables for X-ray Crystallography", Vol. IV, The Kynoch Press, Birmingham, England, Table 2.2 A (1974).

(9) Creagh, D. C. \& McAuley, W.J .; "International Tables for Crystallography", Vol C, (A.J.C. Wilson, ed.), Kluwer Academic Publishers, Boston, Table 4.2.6.8, pages 219-222 (1992).

(10) Creagh, D. C. \& Hubbell, J.H..; "International Tables for Crystallography", Vol C, (A.J.C. Wilson, ed.), Kluwer Academic Publishers, Boston, Table 4.2.4.3, pages 200-206 (1992).

(11) XP: Molecular Graphics program. Part of the SHELXTL Structure Determination Package. Bruker Analytical X-ray Systems Inc.: Madison, WI, (1995-99)

(13) SMART: Area-Detector Software Package, Bruker Analytical X-ray Systems, Inc.: Madison, WI, (1995-99) 